Les inscriptions de Barāqish : apport à la connaissance de l'antique cité de Yathill et du royaume de $\mathrm{Ma}^{\mathrm{C}} \overline{\mathrm{in}}$

Jérémie Schiettecatte

CNRS, UMR 8167 Orient et Méditerranée, Paris

Avec la collaboration de Mounir Arbach

CNRS, UMR 5133 Archéorient, Lyon

\begin{abstract}
The archaeological area of Barāqish, including the extra muros sanctuaries of Shaqab alManașșa and Darb aṣ-Șabī, yielded an exceptional amount of pre-Islamic South Arabian inscriptions. Over 600 texts provide us with an insight into the political, social, and economic history of the ancient kingdoms of Saba' and Ma'in.

This contribution draws up an inventory of the inscriptions from the site and considers this corpus under the angle of ancient toponymy, cultural and architectural history. The examination of the textual content, once put in chronological order, allows for the identification of a set of ancient monuments, the date of their construction, thus highlighting the monumental evolution of the site.

In the meantime, the confrontation of both epigraphy and archaeological data provided by the recent Italian excavations enables us to propose a new chronological sequence of the kings of $\mathrm{Ma}^{\mathrm{C}} \overline{\mathrm{i}} \mathrm{n}$ and to reconsider the date of inscription RÉS 3022 and that of the reign of Abiyada ${ }^{c}$ Yatha' and his son Waqah'īl Riyām.

قدم القطاع الأثري في بر اقث و المناطق المحيطة بها مباثرة، بما في ذلك معابد خارج سور المدينة في شقب المنصة ودرب المبات

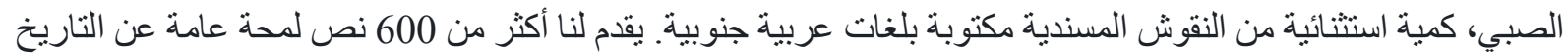

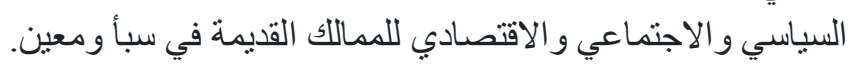

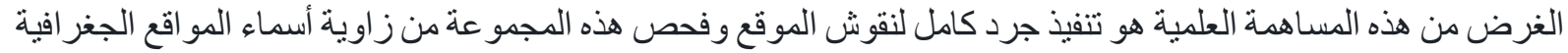

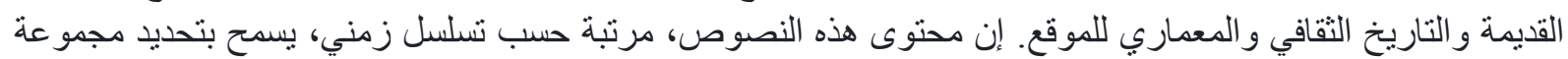

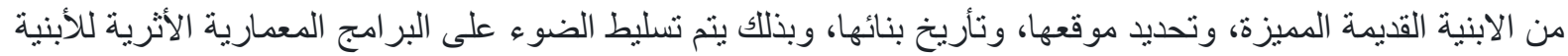
الضخمة التي طبعت تاريخ وحياة الموقع.

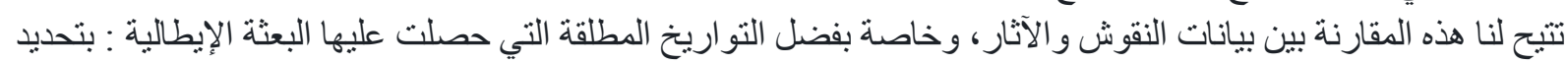

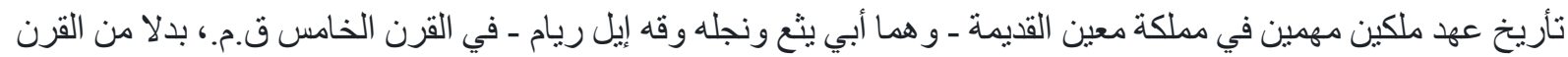
الر ابع ق.م. و هو التأريخ الذي كان معتمدا حتى الآن.
\end{abstract}


Le site de Barāqish et sa zone agricole irriguée ont livré plus de 600 inscriptions sudarabiques. Cela inclue les sanctuaires extra muros de Darb aṣ-Șabī et de Shaqab al-Manașșa, les ruines de Huṣn Āl Șāliḥ et du Wādī Malāhāā. Ces inscriptions sont en majorité de langue ma īnique, une dizaine est en langue saba'ique, une inscription enfin est une bilingue gréco-latine.

A partir de ce corpus et d'études antérieures ${ }^{1}$, le propos consistera à apporter quelques éclairages sur la toponymie antique de la ville et de ses environs, sur son histoire économique, sociale, administrative et politique, sur ses réalisations architecturales et plus largement sur son inscription dans l'histoire des royaumes sudarabiques.

En outre, le corpus complète notre connaissance de la succession des souverains du royaume de Ma`īn. Une restitution des successions dynastiques est présentée en Annexe 4 de cette contribution.

Les bornes chronologiques de cette approche sont fixées par les inscriptions elles-mêmes, du $8^{\mathrm{e}}$ siècle avant au $3^{\mathrm{e}}$ siècle de l'ère chrétienne. Pour les périodes antérieures, on se reportera aux contributions de F. Fedele dans ce volume (voir aussi De Maigret 2010 ; Fedele 2010). Concernant l'histoire du site à l'époque islamique et les sources relatives à cette occupation, une présentation synthétique en a été faite par Ch. Robin (De Maigret \& Robin 1993 : 491-93).

\section{Inventaire épigraphique}

Le corpus épigraphique de Barāqish et de ses environs est exceptionnel par sa quantité et son contenu. Pour les besoins de cette contribution, 611 inscriptions ont été recensées (sigles et table de concordance en Annexe 1). Rares sont les sites sudarabiques qui excèdent un tel nombre (Ma'rib, Raybūn, Hayd ibn 'Aqīl). Parmi ces derniers, seul Ma’rib rivalise par la variété du contenu (religieux, social, économique, politique, historique).

Nous devons en premier lieu ce recueil aux prospections épigraphiques dirigées par :

- Joseph Halévy en 1870 (Halévy 1872);

- Eduard Glaser qui y envoie des émissaires en 1888 (D.H. von Müller \& Rhodokanakis $1913: 83,182)$;

- Muhammad Tawfiq en 1944-45 (Nāmī $1954 ; 1955$; 1956 ; 1957) ;

- Ahmad Fakhry en 1947 (Fakhry 1951);

- Petr Grjaznevich (Bauer \& Lundin 1998) ;

- Jürgen Schmidt en 1977 (W.W. Müller 1982).

Des prospections épigraphiques plus systématiques furent entreprises par la Mission Archéologique Française en République Arabe du Yémen (MAFRAY) sous la conduite de Ch. Robin, à partir de 1976 (Robin 1979a ; 1979b ; 1987 ; Robin et al. 1988), puis la Mission Archéologique Italienne en République du Yémen (MAIRY) sous la conduite de G. Gnoli et A. de Maigret à partir de 1986 (Gnoli \& Robin 1992 ; Gnoli 1993).

Les fouilles sur le site par la mission archéologique italienne à partir de 1989, sous la direction d'A. de Maigret, ont livré des inscriptions supplémentaires trouvées en contexte religieux (De

\footnotetext{
${ }^{1}$ Pour n'en citer que les principales : (Agostini 2012; 2015 ; Antonini \& Agostini 2010 ; De Maigret \& Robin 1993 ; Gnoli \& Robin 1992 ; Robin 1979a ; 1987 ; 1998 ; Robin et al. 1988 ; Robin \& De Maigret 2009).
} 
Maigret \& Robin 1993 ; Agostini 2011 ; 2015) et funéraire (Antonini \& Agostini 2010). S’y ajoutent les inscriptions publiées par A. Agostini dans ce volume.

Une part significative des textes relevés n'a pas encore fait l'objet d'une publication. Tous ne nous sont pas connus et cette présentation ne prétend pas à l'exhaustivité. Les paragraphes suivants présentent les grands ensembles du corpus.

\subsection{Barāqish}

\subsubsection{Rempart}

297 inscriptions, le plus souvent fragmentaires, ont été relevées sur le rempart de Barāqish dont 281 par la MAFRAY, numérotées B-M 1 à B-M 280². La moitié de ces textes était déjà connue par les prospections antérieures et publiée.

Un premier ensemble de textes fut réuni dans le Répertoire d'Épigraphie Sémitique (RÉS 29293060, RÉS 3535, RÉS 4224). G. Garbini les a republiés et complétés de textes additionnels signalés lors des prospections 'Tawfiq' et 'Grjaznevich' (Garbini 1974) : numérotation

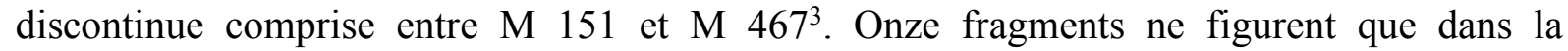
publication de la prospection 'Grjaznevich'4. Un texte, Robin-Barāqish 80, est publié à part (Robin 1979b : 193).

Sept inscriptions trouvées en fouille au pied de la courtine 44-45 du rempart sont publiées dans ce volume : Y.03.B.R44-45.1 à 3 et Y.04.B.T45.1 à 4.

$\mathrm{Au}$ moins 136 textes restent inédits. Une monographie des inscriptions du rempart est en préparation sous la responsabilité de M. Arbach, F. Bron et I. Gajda.

\subsubsection{Temple intra muros de Nakrah}

Soixante-quatorze inscriptions provenant des fouilles du temple de Nakrah ont été portées à notre connaissance. Ch. Robin mentionne 95 inscriptions en incluant les fragments les plus modestes (De Maigret \& Robin 1993 : 458). Treize ont été publiées par Ch. Robin (De Maigret \& Robin 1993), une par Gh. Gnoli (Gnoli 1996), une par A. Agostini (Agostini 2012). Les autres restent inédites.

Les sigles commencent par la séquence Y.90.B.A..., Y.92.B.A... et Y.03.B.A...

\subsubsection{Temple intra muros de 'Athtar dhū-Qabḍ}

Vingt-six inscriptions provenant des fouilles du temple de 'Athtar dhū-Qabḍ ont été portées à notre connaissance. Leurs sigles commencent par la séquence Y.04.B.B..., Y.05.B.B... ou Y.06.B.B...

Trois ont été publiées par A. Agostini : Y.05.B.B.12 et 13 (Agostini 2011) et Y.05.B.B.16 (Agostini 2015). Une quatrième restée inédite, Y.05.B.B.25, fut présentée par A. Agostini lors du colloque $17^{e}$ Rencontres Sabéennes, tenu à Paris en juin 2013. Vingt-deux sont publiées dans le présent volume.

\footnotetext{
${ }^{2}$ Cela inclut trois numéros bis (B-M 19 bis, 23 bis, 211 bis) et deux regroupements (B-M $247+255$ et B-M $26+$ 23).

${ }^{3}$ M 151-158, M 160-161, M 163-174, M 177-201, M 210-220, M 222-233, M 236-270, M 272-283, M 347, M 378, M 404-406, M 410-444, M 446, M 448-449, M 466-467.

${ }^{4}$ Gr 251, 253, 274, 278, 287, 299, 301, 308, 325-326 (Bauer \& Lundin 1998 : 90-110)
} 


\subsubsection{Barāqish, reste du secteur intra muros}

A l'intérieur des remparts de Barāqish, hormis les deux temples, 50 inscriptions ont été relevées par la MAFRAY. Elles portent le sigle B-Int 1 à 50. Seules cinq d'entre elles sont connues de prospections antérieures (B-Int 4, 10, 18, 19 et 21) et publiées : respectivement M 208, M 426, M 204, M 207 et M 206 (Garbini 1974).

On peut ajouter à ce groupe de textes 16 inscriptions relevées par les prospections épigraphiques antérieures à 1976, republiées par G. Garbini (Garbini 1974) ${ }^{5}$, ainsi que cinq inscriptions de la prospection 'Grjaznevich' : Gr 254, 280, 286, 300, 322 (Bauer \& Lundin 1998).

La MAIRY a relevé quatorze inscriptions, non publiées, dont le sigle comporte la séquence Y.90.B..., Y.90.B.ext... et Y.92.B... Un brûle-encens inscrit (Y.86.BAR/13) a été publié (Antonini 1988).

\subsubsection{Barāqish, secteur extra muros $\mathrm{C}$ - sondage}

Onze fragments d'inscriptions proviennent du sondage extra muros réalisé au pied du bastion 7. Ce sont pour neuf d'entre eux des fragments de scellements. Tous sont publiés par A. Agostini dans ce volume avec un sigle commençant par la séquence B.06.C.O... auxquels il faut ajouter Y.06.B.C.1.

\subsubsection{Barāqish, secteur extra muros D - nécropole}

Quarante-cinq inscriptions, principalement des stèles funéraires, ainsi que deux brûle-parfums et une table d'offrande, ont été exhumées lors de la fouille de la nécropole. Elles portent les sigles commençant par les séquences B.05.D.O., B.06.D.O., MAIRY.05 et MAIRY.06. Elles sont publiées dans un ouvrage consacré à la nécropole (Antonini \& Agostini 2010).

On peut ajouter à cet ensemble une stèle funéraire inscrite, Antonini Stela 2, trouvée dans le temple de Nakrah mais provenant vraisemblablement de la nécropole (Antonini 2005).

\subsection{Darb aṣ-Ṣabī}

Trente-et-une inscriptions ont été relevées dans le sanctuaire extra muros de Darb aṣ-Ṣabī, à l'ouest de Barāqish, lors des prospections de la MAFRAY. Elles portent le sigle MAFRAYDarb aṣ-Șabī 1 à 32 et ont toutes été publiées (Robin et al. 1988) ${ }^{6}$.

\subsection{Shaqab al-Manaṣṣa}

Dix-neuf inscriptions ont été relevées dans le sanctuaire extra muros de Shaqab al-Manașșa, au sud-ouest de Barāqish : une lors de la prospection 'Halévy' (Hal 484), quatorze par la MAFRAY (MAFRAY-ash-Shaqab 1 à 14) et quatre par la MAIRY (Y.86.SHQ 1, Y.90.SHQ 2, Y.92.SHQ 3 et 4). Toutes ont été republiées dans une monographie consacrée au site avec le sigle Shaqab 1 à 19 (Gnoli 1993).

\footnotetext{
${ }^{5}$ Ce sont les inscriptions M 159, 162, 175-176, 203, 205, 209, 221, 234-235, 270, 305, 439, 445, 447, 450.

${ }^{6}$ MAFRAY-Darb aș-Ṣabī 14, initialement considérée comme une inscription, s'est avérée être une frise de bouquetins.
} 


\subsection{Périmètre irrigué (ad-Darb, Ḥuṣn Āl Șālị̣, Wādī Malāḥā)}

\subsubsection{Darb al-Ashrāf}

Deux inscriptions ont été retrouvées dans les alluvions, à quelques centaines de mètres au sud du village de Darb al-Ashrāf (ou ad-Darb). Elles ont été publiées avec les sigles Y.90.DA 1 et 2 (Gnoli \& Robin 1992).

\subsubsection{Wādī Malāḥā}

Trois inscriptions ont été retrouvées par la MAFRAY en bordure du Wādī Malāhāa ou remployées dans le village de Malāḥā, au sud de Barāqish. Elles ont pour sigle MAFRAYMalāhāa 1 à 3. Les deux premières ont été publiées (Robin 1987 ; 1988) ; la troisième est inédite.

\subsubsection{Hụṣn Āl Șālị̣}

Cinq inscriptions ont été retrouvées par la MAFRAY aux alentours du village de Ḥuṣn Āl Șālih, cinq kilomètres au sud-sud-ouest de Barāqish. Elles ont pour sigle MAFRAY-Ḥuṣn Āl Șāliḥ 1 à 5. Seules les deux premières, de langue saba'ique, ont été publiées (Robin 1987). Les trois autres, en langue ma īnique, sont inédites.

\subsection{Les inscriptions de musées pouvant provenir de Barāqish}

Plusieurs musées comportent dans leurs collections des inscriptions dont la provenance probable est Ma ${ }^{\text {īn }}$ ou Barāqish. Cette provenance est le plus souvent déduite de la langue des inscriptions (toutes sont en ma ${ }^{\text {īnique }}{ }^{7}$ ) et du contenu (mention des rois et divinités de Ma ${ }^{\top} i n$ ). Vingt-huit inscriptions sont ici recensées ${ }^{8}$ :

- $\quad$ Altes Museum de Berlin : M $337=$ RÉS 3403 ;

- Kunsthistorisches Museum de Vienne : M $342=R E ́ S 3458$;

- Musée d'Istanbul : CIH 678 ;

- Musée de Dhamār : DhM 384-385, 388, 391-393, 399);

- Musée militaire de Sanaa : A-20-207 ;

- Musée national de Sanaa : al-Jawf 04.9, 04.10, 04.21-22, 04.28, 04.30-33, 04.44, 04.46, GOAM 314-315, YM 28166, YM 28335, YM 28336, YM 28988.

Il est rare de pouvoir déterminer duquel des sites de Ma ${ }^{i} \mathrm{n}$ ou Barāqish les textes peuvent provenir. Treize proviennent d'un temple de Nakrah or on en trouve un à Ma'īn et deux à Barāqish (intra et extra muros). Concernant les inscriptions des musées yéménites de Sanaa et Dhamār, la provenance de Ma`in apparaît plus probable. Leurs objets proviennent pour la majorité de fouilles clandestines et Ma īn a été très largement pillée tandis que Barāqish a été relativement épargnée. Un second argument, moins décisif, est que les clans et sous-clans mentionnés par ces inscriptions nous sont plus souvent connus dans les inscriptions de Ma ${ }^{c} i n$ que dans celles de Barāqish :

- Gzyn dans DhM 384 et 388 est attesté dans Ma ${ }^{` i ̄ n ~} 80$;

- $\quad H \underline{d}^{\prime} r$ dans al-Jawf 04.33 est attesté dans Ma'īn 1 et 2 ;

- $\quad Y d^{c}$ dans GOAM 315 est attesté dans Ma'īn 6 (l'auteur de ces deux textes pourrait d'ailleurs être une seule et même personne);

\footnotetext{
${ }^{7}$ Pour le texte YM 28988, le doute est permis. Il pourrait être en saba'ique. Rien ne permet de trancher.

${ }^{8}$ Les renvois bibliographiques sont accessibles dans la Digital archive for the study of pre-islamic arabian inscriptions (http ://dasi.cnr.it).
} 
- Seul Mlh dans YM 28336 est absent des textes de Ma'īn et fréquent dans ceux de Barāqish.

Pour le propos de cette contribution, seuls trois textes dont la provenance de Barāqish semble assurée seront retenus :

- $\quad$ M 337 = RÉS 3403 qui évoque un kabīr de Yathill, titre uniquement porté sur ce site ;

- YM 28336 dont l'auteur appartient à la fraction de clan Mlh, dont le nom apparaît à plusieurs reprises à Barāqish, jamais à Ma'īn ;

- YM 28988 dont l'auteur porte la nisba "le Yathillī / de Yathill" (Yt tly) d'après le nom antique de Barāqish (voir « Toponymie et topographie antique »).

\section{Toponymie et topographie antique}

\subsection{Barāqish, le nom moderne}

Le toponyme Barāqish n'est pas unique au Yémen. L'index toponymique geonames.org recense trois localités ainsi nommées dans les gouvernorats de 'Amrān, Ibb et pour ce qui nous concerne, de Ma'rib, en limite méridionale de la vallée du Jawf.

Dans le Jawf, le toponyme Barāqish (Ar. barqash "multicolore") n'apparaît qu'à la période islamique (al-Hamdān̄̄, al-Iklīl 8 : 64-66 ; Yāqūt, Mu jam al-buldān, 1 : 535). La plus ancienne mention de Barāqish en tant que ville pourrait se trouver dans l'anthologie de la poésie d'alAșma ${ }^{c}$ (v. 740-828), dans un poème attribué à 'Amrū b. Ma'add Yakrub (al-Aṣma i iyyāt, 172). Plusieurs explications ont été données à l'origine de ce nom. Le proverbe arabe « Barāqish nuit à sa propre famille » ('alā ahlihā tajnī Barāqish) trouve son origine dans plusieurs légendes qui se réfèrent tantôt à une femme, tantôt à une chienne multicolore, du nom de Barāqish (Heinrichs 1998). L'une de ces légendes nous est rapportée par al-Hamdānī (al-Iklīl $8: 65$ ) et serait à l'origine du nom du site. La légende raconte qu'alors qu'ils étaient assiégés, les habitants d'une forteresse se ravitaillaient en eau à une citerne située hors les murs en empruntant un tunnel. La présence du tunnel fut révélée aux assaillants par une chienne nommée Barāqish qui, pour aller boire à la citerne, en émergea en aboyant. Les assaillants empruntèrent le tunnel et prirent la forteresse. Celle-ci porte depuis le nom de la chienne, Barāqish.

Selon Yāqūt (Mu`jam al-buldān, 1: 535), ce sont simplement les fleurs multicolores qui parsèment le site qui lui valent ce nom.

\subsection{La ville antique : $Y \underline{t} l$}

Dans l'antiquité, le site de Barāqish était nommé Yathill ( $\underline{\underline{t}} l)$, au moins jusqu'au $3^{\mathrm{e}}$ siècle de l'ère chrétienne. Le toponyme est mentionné dans les inscriptions sudarabiques de manière isolée ou dans la locution hgrn Yt tl, "la ville de Yathill" (Ja 643, M 177, M 185, M 202, M 242, etc.). On retrouve également Yathill dans la locution $s^{2 c} b(h) M^{c} n^{(m)} w$ - $(\underline{d}-) Y \underline{t} l$, « la tribu de $\mathrm{Ma}^{{ }^{\top} \bar{i}} \mathrm{n}^{(\mathrm{um})}$ et de (dhū-)Yathill » (M 29, M 236, M 247, 250, etc.). Cette locution désigne les deux composantes du royaume de Ma'īn : la tribu de Ma'īn d'une part, avec ou sans mīmation, et la tribu de Yathill $(Y \underline{t} l)$ / « celle/celui de Yathill » $(\underline{d}-Y \underline{t} l)$ d'autre part. La présence fréquente, mais non systématique, de la préposition $\underline{d}$ - devant Yathill incite à voir dans cette tribu l'ensemble des habitants de la ville et de ses environs immédiats. 
La nisba ytly, le Yathillī, apparaît à une seule reprise, vers le $7^{\mathrm{e}}$ siècle av. J.-C. (YM 28988 : 'b'ns' bn Lhy ${ }^{\prime} t$ bn $\left.S b h y d^{c} Y t l y\right)$, sans que l'on puisse dire si elle est dérivée du nom de la tribu ou de la ville.

D.H. von Müller a très tôt reconnu dans 'A $\theta \rho o v \lambda \alpha$ (Strabon, Geogr. 16, 4, 24) et 'A $\theta \lambda$ ov $\lambda \alpha$ (Dion Cassius LIII, 29) le nom de Yathill (Müller 1896). Dans les deux sources grecques, il s'agit d'une ville prise sans coup férir par l'armée romaine d'Ælius Gallus (26 av. J.-C.) sur la route

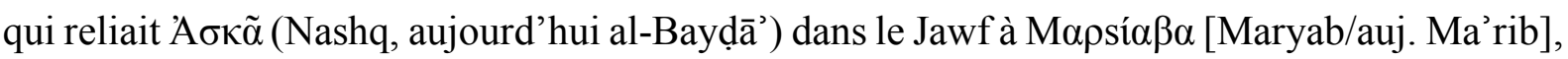
capitale sabéenne. Si l'on note une légère distorsion du nom dans sa transcription grecque, le contexte géographique ne laisse que peu de doute sur l'identification.

J. Pirenne faisait par ailleurs remarquer que le grec 'A $\theta \rho o v \lambda \alpha$ / 'A $\theta \lambda$ ov $\lambda \alpha$ rend vraisemblablement la vocalisation du nom : Yathul (Pirenne 1961 : 105-6). Bien que cette hypothèse soit convaincante, on conservera par commodité la vocalisation Yathill, dont l'usage s'est imposé.

\subsection{L'oasis : Dyt et $S^{2} m m$}

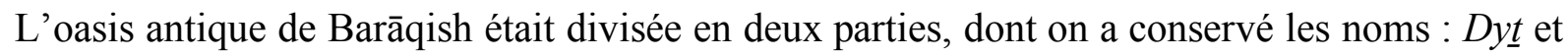
$S^{2} m m$. Elles sont désignées comme les deux plaines irriguées (dhby) de Yathill dans l'inscription sabéenne RÉS 3943 (fin $7^{\mathrm{e}}$ - début $6^{\mathrm{e}}$ siècle av. J.-C.). Il fut un temps envisagé qu'il s'agisse là de noms communs pour « l'aval » et « l'amont» (Robin 1987 : 165). Toutefois, on trouve ces deux noms mentionnés au $7^{\mathrm{e}}$ siècle av. J.-C. dans l'inscription Shaqab 2 comme objets d'une dédicace, à côté du nom des divinités 'Athtar Shariqān et 'Athtar dhū-Yahriq. Il faut en déduire soit que les noms des deux parties constitutives de l'oasis étaient hérités de ceux d'un couple divin, soit que les deux territoires se voyaient conférer une forme de sacralité.

Si l'étude géomorphologique de l'oasis de Barāqish a montré l'existence de deux secteurs distincts (Marcolongo \& Palmieri in De Maigret et al. 1986 : 461-64 ; Marcolongo 1997), il ne s'agit vraisemblablement pas des deux oasis connues par l'épigraphie. Ces dernières apparaissent contemporaines tandis que celles distinguées par B. Marcolongo ont été utilisés à deux périodes successives et relèvent d'une adaptation aux changements des conditions hydrographiques. Le secteur plus ancien était localisé à l'ouest et au sud-ouest de Barāqish ; le second n'aurait été mis en eau qu'à la suite d'une modification du tracé des paléo-cours du Wādī Majzir. F. Fedele associe cet événement à l'épaisse accumulation éolienne observée dans le sondage hors-les-murs (Sand YS), épisode daté vers le milieu du $7^{\mathrm{e}}$ siècle av. J.-C. (Fedele $2010: 118-19)$.

La résolution chronologique n'est pas suffisamment précise pour dater les inscriptions Shaqab 2 et $R E S 3943$ ainsi que l'événement géomorphologique qui entraîne l'abandon d'une zone irriguée au profit d'une seconde. A titre d'hypothèse et en s'accordant avec les dates proposées, on peut envisager la succession des événements comme suit : milieu du $7^{\mathrm{e}}$ siècle av. J.-C., abandon d'une zone irriguée au sud-ouest de Barāqish et redéploiement de l'oasis vers le sudest ; ce nouveau périmètre irrigué se scinderait en deux oasis dont on peut imaginer que chacune disposait de son propre système d'adduction en eau : Dyt et $S^{2} m m$. D'après Shaqab $2\left(7^{\mathrm{e}}\right.$ siècle av. J.-C.), on ne peut exclure que les noms de ces deux oasis soient hérités d'un couple de divinités locales mineures. Les deux oasis sont ravagées dans le courant du $6^{\mathrm{e}}$ siècle av. J.-C. (RÉS 3943). 
Les textes ont livré quelques toponymes supplémentaires qui se trouvaient dans le secteur irrigué de Barāqish mais ne peuvent être localisés de manière précise :

- $\underline{d}-B^{\prime} r n$ : secteur agricole dans lequel un puits est creusé (Shaqab 16);

- $\underline{d}$-Hrs : propriété agricole (qny) de Wqh'l Rym, roi de Ma'īn (Shaqab 18);

- $\underline{d}-H z w t$ : parcelle irriguée ( $m \underline{d} r)$ (MAFRAY-Malāhāā 1)

- $\underline{d}-N^{2} d m$ : secteur agricole (Y.90.DA 1 et 2).

- $\quad \underline{d}$-Ynzm : secteur agricole (Y.90.DA 2)

- $\quad \underline{d}$-Zwr : palmeraie (nhl) (MAFRAY-Ḥuṣn aṣ-Șāliḥ 3) ;

- $\quad \underline{d} t-R m l n$ : propriété agricole (qny) de Wqh'l Rym roi de Ma'̄n (Shaqab 18).

Certains pourraient tirer leur nom du patronyme ou du lignage de leur propriétaire. Zwr par exemple est un nom de lignage connu par ailleurs (as-Sawdā’ 91).

\subsection{Les sanctuaires extra muros : Yhrq et $\boldsymbol{F t}^{\mathrm{c}} \boldsymbol{n}$}

Les environs de Barāqish comptent deux sanctuaires extra muros. Le premier, localisé à Shaqab al-Manașșa, environ 2,5 km en direction du sud-ouest Barāqish, borde la rive gauche du Wādī al-Khawr. Consacré au dieu 'Athtar dhū-Yahriq, G. Gnoli voit dans l'épithète Yhrq le nom antique du sanctuaire (Gnoli 1993 : 15-16). L'élément le plus déterminant est l'inscription Shaqab 8 qui mentionne " 'Athtar dieu de Yahriq » (1. 2 : ' $t$ tr 'l Yhrq). L'inscription sabéenne du Jabal Riyām RÉS 4176, bien que géographiquement éloignée, pourrait évoquer ce sanctuaire dans la formule "'Athtar et les dieux de Yahriq» (1. 9: 'ttr w-'l'lt b-Yhrq) (voir également « 5.2.5 - Yhrq, temple de 'Athtar à Shaqab al-Manașșa).

Le second sanctuaire extra muros se trouve à Darb aṣ-Ṣabī, moins de deux kilomètres à l'ouest de Barāqish. Il s'agit d'un vaste enclos sacré $(\mathrm{mhrm})$ délimité par neuf bornes et comprenant une quarantaine d'édifices. Le nom antique de ce périmètre sacré, $F \underline{t}^{\dagger} n$, nous est connu par l'inscription MAFRAY-Darb aṣ-Șabī 1 (Robin et al. 1988 : 92) (voir également « 5.2.6-Fț $n$, sanctuaire de Nakraḥ à Darb aṣ-Ṣabī).

\subsection{Les villes périphériques : $M h f d n, N^{c} m n, R d^{c}$ et $Y^{c} d$}

L'inscription ma īnique Shaqab 1, datée du $7^{\mathrm{e}}$ siècle av. J.-C., rapporte les offrandes faites par les auteurs du texte dans le temple de Wadd à Qarnā (auj. Ma'īn), Yathill (auj. Barāqish) et dans les bourgades (hgrn) de $M h f d n, N^{c} m n, R d^{c}$ et $Y^{c} d$. Nous avons vraisemblablement ici les noms de quatre agglomérations du territoire du royaume de $\mathrm{Ma}^{\text {‘inn }}$ qu'il faut chercher dans les environs de Ma`īn et Barāqish. Aucun de ces toponymes n'est précisément localisé.

\section{$4 \quad$ Histoire de la cité antique de Yathill}

L'histoire de l'antique cité de Yathill a été évoquée à travers plusieurs études (Robin 1979a ; 1984 ; Robin 1987 ; Gnoli \& Robin 1992 ; De Maigret \& Robin 1993 ; Gnoli 1993 ; Robin \& De Maigret 2009; De Maigret 2010; Fedele 2010; Schiettecatte 2011 : 51-57). On en rappellera ici les principaux éléments.

Pour commencer, Yathill est une ville de frontière. Elle se trouve en bordure méridionale de la vallée du Jawf, à la frontière nord du royaume de Saba' et au contact des principautés du Jawf. Au cours du I ${ }^{\mathrm{er}}$ millénaire av. J.-C., la ville se trouve ainsi successivement intégrée dans le 
royaume de Saba', puis dans le royaume dominant de la vallée du Jawf, Ma'īn, avant d'être peuplée par des membres de la tribu d'Amīr, qui s'établit dans le Jawf à partir du $2^{\mathrm{e}}$ siècle av. J.-C. Ces changements politiques eurent une incidence sur la vie sociale, culturelle et religieuse de la cité.

\subsection{Une cité sabéenne (jusqu'au $7^{\mathrm{e}}$ siècle av. J.-C.)}

Jusqu'au milieu du $7^{\mathrm{e}}$ siècle av. J.-C., un faisceau d'éléments montre l'inscription de la ville de Yathill dans le royaume de Saba'.

La langue des inscriptions est le saba'ique (YM 28988, MAFRAY-Ḥuṣn Āl Ṣālị 1 et 2, MAFRAY-Malāhā̄' 2, Y.90.DA 1 et 2, Shaqab 15). Toutes proviennent du secteur irrigué et des sanctuaires extra muros. Toutes sont de graphie A (pal.Pi.) $)^{9}$ et datent de la fin $8^{\mathrm{e}}$-début $7^{\mathrm{e}}$ siècles av. J.-C.

Le panthéon, lui aussi sabéen, inclut la triade divine 'Athtar, Almaqah et dhat-Himyam (MAFRAY-Ḥuṣn Āl Șāliḥ 2) ou Almaqah et 'Athtar (MAFRAY-Malāḥā' 2).

Le calendrier est sabéen, avec la référence faite aux mois de $\underline{d}-K s^{2} b m$ (Y.90.DA 1) et $\underline{d}-S^{I} h r$ (Y.90.DA 2).

Une inscription est datée par un magistrat (prêtre) éponyme de Saba': Y.90.DA 2 qui mentionne 'lrm fils de $M l k s^{I} m^{c}$, nom qui apparaît dans la liste des éponymes (Ja 2848y) du Jabal Balaq al-Janūbī, non loin de Ma’rib (Gnoli \& Robin 1992 : 97). Y.90.DA 1 mentionne un second magistrat éponyme de Saba', $S^{I} m h k r b$ fils de $Y h q m$, inconnu par ailleurs.

Enfin, la reconnaissance du mukarrib sabéen comme souverain apparait dans Shaqab 15, où un certain ' $S^{l} d y \underline{t}{ }^{c}$ se définit comme serviteur du souverain sabéen $S^{l} m h^{`} l y$.

Le sondage stratigraphique sous les niveaux du temple de Nakrah dans le secteur intra muros a révélé une occupation continue du $13^{\mathrm{e}}$ au $7^{\mathrm{e}}$ siècle av. J.-C. et a livré, dans les niveaux des trois derniers siècles de cette occupation, un assemblage céramique homogène et caractéristique des productions attestées sur les sites sabéens contemporains, en particulier la céramique carénée rouge à brunissage (De Maigret 2010 : 85-87).

$\mathrm{Au}$ cours de cette période sabéenne, trois institutions ou offices apparaissent dans les inscriptions Huṣn '1 Șālih 1 et Y.90.DA 2 : le conseil de Yathill ( $\left.m s^{3} w d Y \underline{t} l\right)$, le chef de Yathill $(k b r Y \underline{t} l)$ et un contrôleur de l'irrigation $(m d r r)$. G. Gnoli et $\mathrm{Ch}$. Robin proposent de voir dans le kabìr un représentant du pouvoir sabéen tandis que le conseil réunit les membres influents de la tribu locale, Yathill ${ }^{10}$, chefs des principaux clans ou lignages. Le kabīr est investi d'un pouvoir exécutif (Gnoli \& Robin 1992 : 97). Il est également chargé, comme édile, de la gestion du périmètre irrigué et par-là même de l'approvisionnement de la ville de Yathill. La tribu de Yathill bénéficie d'une certaine autonomie, puisque l'invocation aux divinités sabéennes n'est pas systématique et que le droit de légiférer lui incombe au moins partiellement.

\footnotetext{
${ }^{9}$ L'abréviation Pal.Pi. désigne les styles paléographiques définis par J. Pirenne (1956). L'abréviation Pal.Av. désigne ceux définis par A. Avanzini (2004).

${ }^{10}$ Le nom de la tribu apparaît distinctement dans plusieurs inscriptions sabéennes des $8^{\mathrm{e}}-7^{\mathrm{e}}$ siècles du site et de ses environs : dans l'invocation finale de MAFRAY-Ḥuṣn Âl Șāliḥ 2; dans les locutions « conseil de Yathill » $\left(m s^{3} w d Y \underline{t} l\right)$ et «chef de Yathill » $(k b r \quad Y \underline{t} l)$ dans MAFRAY-Ḥuṣn Āl Șāliḥ 1 et Y.90.DA 2; et dans la nisba Yathillite (Y $\underline{\underline{t} l y}$ ) dans l'inscription YM 28988.
} 
L'unique réalisation majeure du pouvoir sabéen à Yathill est la construction d'un premier rempart à l'initiative du mukarrib sabéen Karib'īl Watār fils de Dhamar'alī (RÉS 3946), vers 680 av. J.-C.

\subsection{L'alliance des tribus de Ma'in et Yathill (v. 670-650 av. J.-C.) et les relations ultérieures avec Saba'}

L'existence d'un royaume de $\mathrm{Ma}$ 'īn a longtemps été niée avant le $7^{\mathrm{e}}$ siècle av. J.-C., notamment par l'absence de mention du royaume dans les récits des hauts faits des mukarribs sabéens des $8^{\mathrm{e}}-7^{\mathrm{e}}$ siècles av. J.-C. (DAI Șirwāḥ 2005-50, RÉS 3945 et 3946). La découverte récente d'inscriptions royales minéennes datées de ces périodes anciennes a substantiellement modifié cet état de fait : Ma īn existait en tant qu'entité politique dès le $8^{\mathrm{e}}$ siècle av. J.-C. (Robin et al. 2007 ; Schiettecatte 2011 : 61-62 ; Arbach 2011 ; Arbach \& Rossi 2012 ; Arbach 2018).

Le silence des textes sabéens pourrait s'expliquer par une alliance entre Ma'īn et Saba' aux $8^{\mathrm{e}}$ $7^{\mathrm{e}}$ siècles av. J.-C. Trois éléments vont dans ce sens :

- La mention de la divinité dhat-Himyam dans l'inscription Ma ${ }^{\text {īn }} 109$, v. le $7^{\mathrm{e}}$ siècle av. J.-C., une divinité dont le culte est principalement attesté en domaine sabéen ;

- La mention de la fraternité entre 'byd $Y f s^{2}$ fils de $N b t^{\prime} l$ roi de Ma īn d'une part et $Y d^{(`} l$ [Drh $]$ mukarrib de Saba' d'autre part, vers le $7^{\mathrm{e}}$ siècle av. J.-C.

- La dédicace du roi de Ma'īn $W q h^{\prime} l$ fils de 'lyf $f^{\text {c }}$ dans le temple de Wadd dhu-Masma im (Schm/Samsara 3 ) à proximité de Ma'rib vers le milieu du $7^{\mathrm{e}}$ siècle av. J.-C.

$\mathrm{Au}$ sein de cette alliance vraisemblable entre $\mathrm{Ma}^{\text {'īn }}$ et Saba', on voit la tribu de Yathill quitter la sphère sabéenne pour se rallier à Ma ${ }^{\mathrm{i}} \mathrm{n}$ dans le courant $\mathrm{du} 7^{\mathrm{e}}$ siècle av. J.-C.

Cet événement intervient tôt dans le courant du $7^{\mathrm{e}}$ siècle av. J.-C. Le plus ancien document attestant cette bascule est l'inscription Shaqab 6 datée par la graphie de la première moitié du $7^{\mathrm{e}}$ siècle av. J.-C. (Pal.Pi. A4-B1). Le texte mentionne un membre du clan $G b^{\prime} n$, clan dominant de la tribu de Ma'īn, prêtre $\left(r s^{2} w\right)$ de la divinité tutélaire de la tribu de Yathill, 'Athtar dhuYahriq, qui dédie son inscription aux dieux de Ma'īn et de Yathill à l'époque de 'lyf Rym (I) roi de Ma'īn.

Peu de temps après, l'inscription Shaqab 1, datable du $7^{\mathrm{e}}$ siècle av. J.-C. (Pal. Pi. B2-B3), évoque la réalisation de sacrifices au profit des divinités du royaume de Ma'īn : 'Athtar dhuQabḍ et Wadd. L'inscription commémore des célébrations réalisées conjointement dans l'ensemble du royaume de Ma'īn : au torrent de Wadd $(\dot{g} y l \mathrm{Wdm})$, dans les villes du royaume : Qarnā, Yathill, $M h f d n, N^{c} m n, R d^{c}, Y^{c} d$ et dans le sanctuaire Yahriq (Shaqab al-Manașșa).

Cette fédération des tribus de $\mathrm{Ma}$ 'īn et Yathill reste proche du pouvoir sabéen comme le montre la dédicace de $W q h^{\prime} l$ fils de ' $l y f^{\star}$ roi de Ma'īn dans le temple sabéen de Wadd dhu-Masma'im, au $7^{\mathrm{e}}$ siècle av. J.-C. Cette proximité est également suggérée par la culture matérielle. La production céramique présente un répertoire formel et des caractéristiques techniques héritées des productions dites « sabéennes » antérieures, en dépit d'une perte de qualité (Fedele 2010 : 126).

Qualifier l'occupation de Yathill de la fin du $7^{\mathrm{e}}$ siècle av. J.-C. de « Later Sabaean Phase » sur la base de la culture matérielle porte toutefois à confusion dans le sens où la ville et sa population apparaissent dès lors clairement comme une composante majeure du royaume de Ma'īn. 
A compter de cette date et jusqu'au $1^{\text {er }}$ siècle av. J.-C., l'insertion de Yathill dans le royaume de Ma'in se manifeste par :

- L'usage de la langue ma īnique ;

- L'adoption du panthéon de Ma'īn avec la triade 'Athtar dhu-Qabḍ, Wadd et Nakrạ ;

- L'association des « dieux (des villes) de Qarnā et Yathill» (B-M 115 : 'l'lt Qrnw w$Y \underline{t} l)$;

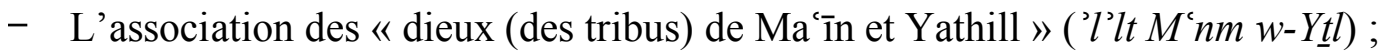

- La reconnaissance de l'autorité des rois de Ma'īn et de l'assemblée de Ma`īn (RÉS 3013 : $m l k y M^{\mathrm{c}} n$ w-ms ${ }^{3} w d M^{\mathrm{c}} n^{m}$ ).

- L'insertion de la tribu de Yathill au sein de « la tribu de Ma'īn et Yathill » $\left(s^{2^{c}} b M^{c} n w\right.$ $Y \underline{t} l)$;

- L'adoption de la structure clanique du royaume de Ma`īn (Robin 1984).

Tout au long de cette période, les rapports avec le royaume de Saba’ oscillent entre des périodes d'alliance et d'affrontement :

- Alliance $-2^{\mathrm{e}}$ moitié du $7^{\mathrm{e}}$ siècle av. J.-C. : dédicace d'un roi de Ma`īn, $W q h^{\prime} l$ fils de 'lyf', dans le temple sabéen de Wadd dhu-Masma'im ;

- Affrontement - fin $7^{\mathrm{e}}$-début $6^{\mathrm{e}}$ siècle av. J.-C. : un conflit oppose Saba' à une coalition regroupant Muha'mir, Amīr et Ma'īn et aboutit au siège et à la destruction de la ville de Yathill. L'événement est rapporté dans l'inscription RÉS 3943 vraisemblablement datée

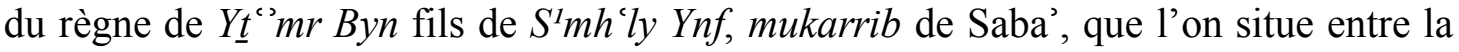
fin du $7^{\mathrm{e}}$ siècle av. J.-C. (Avanzini $2016:$ 133) et le milieu du $6^{\mathrm{e}}$ siècle av. J.-C. (Robin 2016a : 71) ;

- Affrontement - Fin $5^{\mathrm{e}}$ siècle av. J.-C. : sous le règne de 'byd $Y \underline{t}^{c}$, attaque d'une caravane de Ma`īn par la tribu de Saba' (RÉS 3022) dont l'inscription Demirjian 1 semblerait également se faire l'écho (Multhoff 2019);

- Alliance - Fin $5^{\mathrm{e}}$-début $4^{\mathrm{e}}$ siècle av. J.-C. : le roi $W q h^{\prime} l$ Rym fils de 'byd' place la construction d'un réservoir «sous la protection de 'Athtar Shāriqān, des divinités de

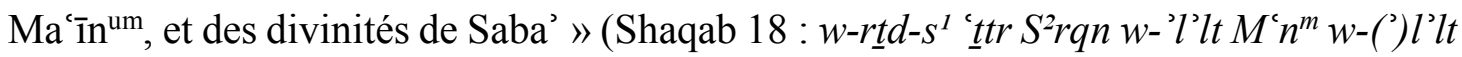
$\left.S^{l} b^{\prime}\right)$;

- Alliance - Fin $5^{\mathrm{e}}$-début $4^{\mathrm{e}}$ siècle av. J.-C. : Un texte contemporain du précédent par la graphie, Shaqab 8, place le temple de 'Athtar dhu-Yahriq sous la protection des divinités de Ma'īn et de 'Athtar dhu-Dhibān or cette divinité n'est attestée qu'en domaine sabéen (Robin 1996 : 64);

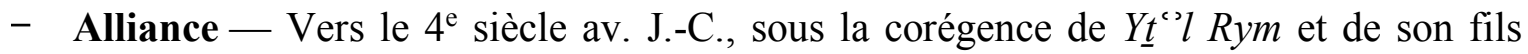
$T b b^{`} k r b$, des membres du sous-clan $D b r$, fraction de $Y l q z$ consacrent leurs travaux aux dieux de Ma'īn et de Yathill ainsi qu'à « tous les dieux, patrons, rois et tribus de Saba’ » (RÉS 2980 bis : $k l^{\prime} l^{\prime} l t w$ - $S^{2} y m h y w$-'mlk $w$-' $\left.S^{2} b S^{\prime} b^{\prime}\right)$.

De cette succession d'événements, il apparaît qu'après une période d'alliance entre Ma'īn et Saba' au $7^{\mathrm{e}}$ siècle av. J.-C., le rapprochement de la tribu de Yathill à celle de Ma'īn puis la prise progressive de contrôle du commerce caravanier par Ma'inn a contribué à l'accroissement des 
tensions entre les deux royaumes, menant à l'assaut de Yathill à la fin $7^{\mathrm{e}}$-début $6^{\mathrm{e}}$ siècle av. J.-

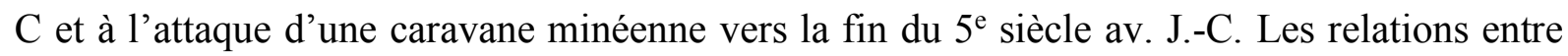
Saba' et $\mathrm{Ma}^{\text {'īn }}$ s'apaisèrent ensuite avec une période d'alliance manifeste dans le courant du $4^{\mathrm{e}}$ siècle av. J.-C.

\subsection{Yathill, cité minéenne ( mi- $^{\mathrm{e}}-\mathrm{1}^{\mathrm{er}}$ siècle av. J.-C.)}

\subsubsection{De la cité moribonde à la monumentalisation urbaine}

La cité de Yathill ne semble guère florissante au moment où elle intègre la sphère minéenne, au $7^{\mathrm{e}}$ siècle av. J.-C. La fouille du secteur extra muros a révélé un épais ensablement de l'ouest du site, postérieur à l'abandon de ce qui apparaît comme le rempart sabéen (wall F4). F. Fedele s'interroge sur un possible lien entre cet événement stratigraphique et les bouleversements qui ont affecté le tracé du cours du Wadi Majzir dans l'antiquité, forçant un déplacement de l'ensemble de la zone agricole (Fedele 2010 : 119). Yathill n'est alors plus fortifiée.

Peu après, l'inscription RÉS 3943 rapporte le siège et le sac de la ville de Yathill et de ses environs :

« Et il assiégea Yathill et dévasta les deux oasis de Yathill, Dyt et $S^{2} m m$, et il incendia et détruisit

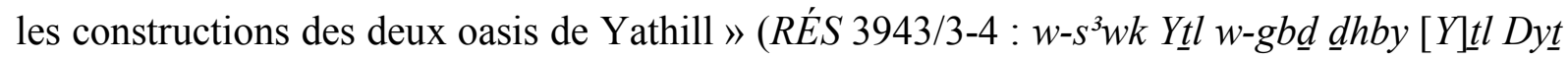
$w-S^{2} m m w$-wft $w$-s $\left.\left.s^{2} \operatorname{tr} m b n(y)\left[\underline{d}^{(4)} h\right](b) y Y \underline{t} l\right]\right)$. Ce n'est qu'à la suite de ces événements que sont entrepris les premiers travaux de monumentalisations de la ville de Yathill avec l'aménagement du temple de Nakrạ̣ et du rempart, entamés au cours du $6^{\mathrm{e}}$ siècle av. J.-C. (voir « 5 - Les monuments de Barāqish » et plus particulièrement « 5.5 - L'activité de construction »).

\subsubsection{Yathill, organisation sociale}

Avec l'intégration au royaume minéen, les institutions de la période sabéenne cèdent la place à d'autres formes de pouvoir. Les décisions émanent du souverain minéen ( $\left.m l k M^{\mathrm{c}} n\right)$ et du conseil de $\mathrm{Ma}^{\mathrm{i}} \overline{\mathrm{i}} \mathrm{n}\left(m s^{3} w d M^{\mathrm{c}} n\right)$, réunissant les notables de la tribu de Ma'īn-et-Yathill (ex. : RÉS 2959, $R E ́ S$ 3013). L'application de leurs décisions semble être à la charge d'un chef/kabìr de Yathill

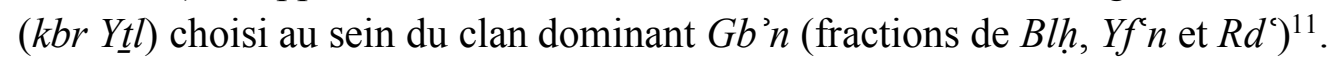

Les habitants de la Yathill minéenne se rattachent alors à la vaste tribu de Ma īn-et-[dhu]Yathill $\left(s^{2 c} b M^{c} n w-[\underline{d}-] Y \underline{t} l\right)$, qui fédère l'ensemble des clans du royaume minéen. Elle se subdivise en clans, eux-mêmes divisés en sous-clans, puis en lignées. Lorsqu'il décline son identité, l'individu mentionne l'un ou l'autre de ces éléments. La parenté ou la lignée est introduite par $b n$, le sous-clan en général par $\underline{d}$ - ou ' $h l$ et le clan par ' $h l$ (Robin 1984).

Les clans et sous-clans attestés à Yathill sont recensés en Annexe 3. Cette liste permet quelques observations.

Les sous-clans attestés à Barāqish ne sont presque jamais attestées à Qarnā ${ }^{-12}$, à de rares exceptions près, toutes issues du clan $G b^{\prime} n$.

\footnotetext{
${ }^{11}$ Un kabīr de Yathill est mentionné dans les inscriptions RÉS 2961 ; RÉS 2939 ; M 419 ; RÉS 3022 ; Y.92.B.A 21+30, Y.03.B.R44-45.3.

${ }^{12}$ Sont exclues ici les inscriptions de Qarnā réunies sous l'appellation de «Liste des hiérodules »; dans ces dernières, les personnes évoquées ne relèvent pas seulement de la population de Qarnā mais de la totalité du royaume de Ma'īn ; ces textes recensent les épouses prises par des commerçants minéens dans les villes et régions partenaires.
} 
De même, rares sont les clans attestés dans les deux villes. Mwqh et Ylqz sont attestés à Qarnā (Ma`în 30 et 15) mais à travers d'autres sous-clans que ceux connus à Yathill.

Cette observation va dans le sens de deux populations relativement distinctes, celle réunie dans la tribu de Yathill d'un côté, celle de la tribu de Ma'īn de l'autre.

La seule exception est le clan $G b^{\prime} n$ et certaines de ses fractions ( $\left.H f n, R d^{c}, S^{2} \underline{t} t m, Z l w m n\right)$ qui apparaissent aussi bien à Qarnā qu'à Yathill.

Parmi les clans s'instaure une hiérarchie au sein de laquelle le clan Gab'ān $\left(G b^{\prime} n\right)$ domine clairement. Outre la charge de chef de Yathill $(k b r Y \underline{t} l)$, on retrouve les membres de ce clan au sein de la congrégation (qhlt) de 'Athtar dhu-Yahriq (Y.92.B.A $21+30$ ), parmi les prêtres $\left(r s^{2} w\right)$ de 'Athtar dhu-Yahriq (Shaqab 6), parmi les chefs des caravaniers de Ma'inn (RÉS 3022 : $\left.k b r M^{c} n m s ̦ r n\right)$. Ce sont en très grande majorité les membres de ce même clan qui financent les principales réalisations monumentales dans la ville : le réaménagement du temple de Nakrah, la construction du temple de Wadd, celle de sept courtines et de quinze bastions du rempart et celle de quatre résidences (voir « 5.5 - L'activité de construction »). Certains de ses membres prennent le titre d'ami du roi (RÉS 2771), d'autres prennent épouse dans les différentes villes et régions partenaires du commerce caravanier transarabique : Dadān, Yathrib, Ghazzat, Sidon, Hagar/Gerrha, en Égypte et en Ionie ( $\mathrm{Ma}^{c} \bar{i} n 93$ à $\mathrm{Ma}^{c} \bar{i} n$ 98).

Cet ascendant pris par les membres du clan $G b^{\prime} n$ amène à penser que certains sous-clans de Yathill ayant des offices importants et pour lesquels le clan d'appartenance n'est pas connu pourraient appartenir à ce clan aristocratique. On peut en particulier penser au sous-clan $D f g n$

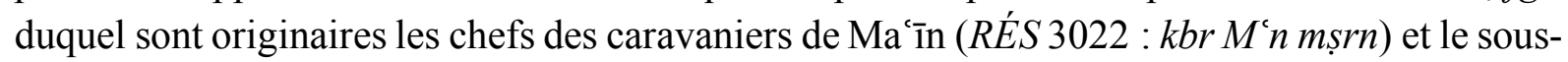
clan de $\dot{G} z r-S^{I} h f n$ au sein duquel on trouve un chef des serviteurs du roi ( $k b r$ ' $\left.d m m l k\right)$, bâtisseur d'un bastion (RÉS 2976) et un chef des maçons et du transport ( $k b r$ grbn w-nqln), bâtisseur du temple Qabḍim (Y.05.B.B.13).

\subsubsection{Yathill, cité caravanière}

La documentation épigraphique minéenne, en particulier celle de Barāqish, reflète l'importance du commerce caravanier transarabique pour l'économie de l'antique Yathill et du royaume de Ma'īn.

Parmi les débouchés commerciaux majeurs des $5^{\mathrm{e}}-4^{\mathrm{e}}$ siècles av. J.-C. figurent les provinces perses du Proche-Orient : l'Égypte, l'Assyrie et la Transeuphratène (Mṣr w- "' $s^{2} r$ w- 'br Nhrn RÉS 2930, RÉS 3022).

Les membres des sous-clans de Yathill s'établissent dans des comptoirs commerciaux fondés dans les principales oasis qui ponctuent le tracé des pistes caravanières (tableau 1). 


\begin{tabular}{|c|c|c|}
\hline Sous clan & Clan & $\begin{array}{l}\text { Attestation d'un (sous-)clan originaire de Yathill ou lieu d'origine d'une épouse d'un } \\
\text { membre de ces (sous-)clans }\end{array}$ \\
\hline Mlh & $?$ & $\begin{array}{l}\text { Qaryat al-Fāw (Qaryat-Zaydwadd) : Chef }(k b r) \text { des Minéens de Qaryat al-Fāw } \\
\text { al-'Ulā (RÉS 3272) }\end{array}$ \\
\hline$M r n$ & $?$ & $\begin{array}{l}\text { Qaryat al-Fāw (Riyāḍ 302F8) } \\
\text { al-'Ulā (RÉS 3339, RÉS 3700) : Chef (kbr) des Minéens de Dadān }\end{array}$ \\
\hline Rwyn & $?$ & Tamna' (VL 9) : Chef $(k b r)$ des Minéens de Tamna $^{c}$ \\
\hline 'rqn & $?$ & al-`Ulā (RÉS 3608) : Chef $(k b r)$ des Minéens de Dadān \\
\hline [Zhrn] & $G b^{\prime} n$ & Épouse prise à Dadān [al-`Ulā] (Ma īn $93 \mathrm{~A} / 30)$ \\
\hline$H d \underline{d l n}$ & $G b^{\prime} n$ & Épouse prise à Ghazzat [Gaza] (Ma īn 93 B/11) \\
\hline$R d^{c}$ & $G b^{\prime} n$ & 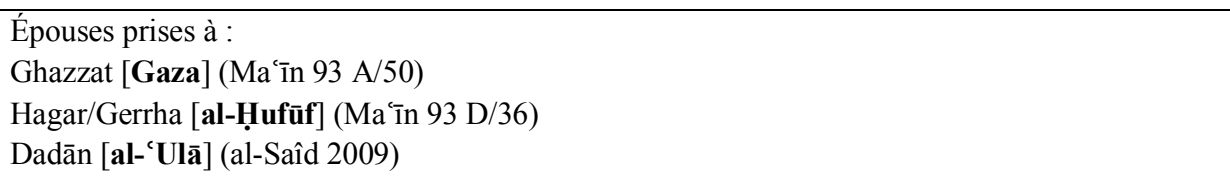 \\
\hline$S^{2} \underline{t} t m$ & $G b^{\prime} n$ & Épouse prise à Yathrib [Médine] (Ma īn 95/10) \\
\hline$Y f^{\prime} n$ & $G b^{\prime} n$ & $\begin{array}{l}\text { al- 'Ulā (Ja 2288, RÉS 3344, RÉS 3346, RÉS 3353) : Chef (kbr) des Minéens de Dadān } \\
\text { Épouse prise à Awsān [wādī Markha] (Ma īn } 93 \text { C/52) }\end{array}$ \\
\hline Zlwmn & $G b^{\prime} n$ & $\begin{array}{l}\text { Madā'in Șāliḥ (RÉS 3708) } \\
\text { Tamnac (VL 9) } \\
\text { Épouses prises à Ghazzat [Gaza] (Ma'īn } 93 \text { C/3, 19) }\end{array}$ \\
\hline$\dot{G} r b t$ & Mwqh & $\begin{array}{l}\text { al-`Ulā (M } 390 ; R E ́ S 3697 ; R E ́ S 3283) \\
\text { Épouse prise à } \boldsymbol{H} \boldsymbol{s}^{2} \boldsymbol{m}(?)\left(\mathrm{Ma}^{\top} \mathrm{i} \text { in } 93 \mathrm{~B} / 30\right)\end{array}$ \\
\hline Zyrn & $M w q h$ & $\begin{array}{l}\text { Madāin Șāliḥ (RÉS 3708) } \\
\text { Fayyūm (RÉS 3427) } \\
\text { Épouses prises en Égypte (Ma īn } 93 \text { C/39-40 ; Ma īn 95/13-14) }\end{array}$ \\
\hline$H \underline{d} d k t$ & Qrn & $\begin{array}{l}\text { Épouses prises à : } \\
\text { Qaryat al-Fāw (Ma`īn } 93 \mathrm{~B} / 36-37) \\
\text { Tml冖 (?) (Ma`īn 95/17) }\end{array}$ \\
\hline$D b r$ & Ylqz & $\begin{array}{l}\text { Qaryat al-Fāw (Riyad 262F8) } \\
\text { Épouse prise dans le Hạ̣ramawt (Ma'īn } 93 \text { A/60) }\end{array}$ \\
\hline \multirow[t]{2}{*}{ Mhd } & Ylqz & Épouse prise à Mo'ab (Ma īn 93 D/31) \\
\hline & Ylqz & $\begin{array}{l}\text { Épouses prises à : } \\
\text { Dhakir (Fontaine-Arbach fig. 26) } \\
\text { Égypte (Ma'īn } 93 \mathrm{~B} / 4 \text {; Ma'īn } 93 \mathrm{C} / 26) \\
\text { Ghazzat [Gaza] }(\mathrm{Ma} \text { 'inn } 93 \mathrm{C} / 43) \\
\text { Tmlh (?) (Ma'īn } 93 \mathrm{~A} / 19)\end{array}$ \\
\hline$S^{\prime} y l$ & 'ly'l & 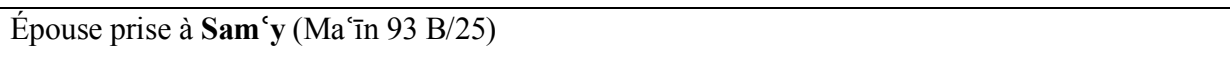 \\
\hline$F^{\prime} m n$ & $\begin{array}{l}\text { 'ly'l } \\
\text { (?) }\end{array}$ & 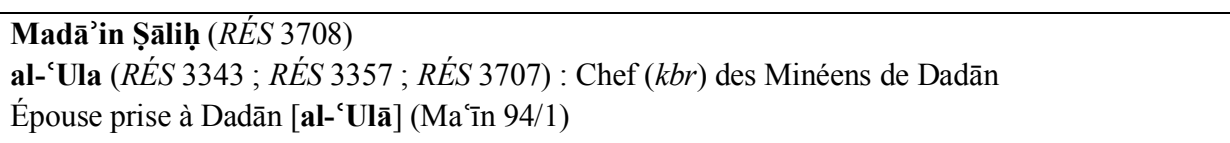 \\
\hline
\end{tabular}

Tableau 1 : Relation des clans / sous-clans de Yathill (actuelle Barāqish) avec l'étranger : attestations hors du royaume de $\mathrm{Ma}$ īn et mariages avec des épouses étrangères. 
A Yathill, cinq clans se partagent l'organisation du commerce transarabique : Gb' $n, M w q h$, Qrn, Ylqz et ' $l y ' l$. Le fonctionnement de ce commerce est coordonné par deux offices : les chefs des caravaniers d'une part ( $k b r M^{\prime} n$ mșrn - RÉS 3022, RÉS 3535) qui se chargent du transport des marchandises; les chefs des communautés minéennes implantées dans les villes étapes majeures le long du tracé des voies caravanières : Tamna' (VL 9), Qaryat al-Fāw (QaryatZaydwadd) et Dadān (actuelle al-'Ulā — Ja 2288, RÉS 3346, RÉS 3608).

Les trois royaumes sudarabiques contemporains de $\mathrm{Ma}^{\mathrm{C}} \mathrm{in}$ apparaissent comme des partenaires relativement constants au cours de la période du $5^{\mathrm{e}}$ au $2^{\mathrm{e}}$ siècle av. J.-C.

Les liens avec le royaume de Saba' sont visibles dans les évocations des souverains et dieux sabéens aux côtés de ceux de Ma'īn dans les textes Shaqab 8, Shaqab 18 et RÉS 2980 bis (voir 4.2 — L'alliance des tribus de Ma īn et Yathill (v. 670-650 av. J.-C.) et les relations ultérieures avec Saba').

On trouve mention de liens manifestes avec le royaume du Hadramawt :

- La présence à Yathill d'habitants originaires du Haḍramawt et de Shabwa sa capitale (B-M 73) ;

- La commémoration du transport de marchandises du Haḍramawt (Y.05.B.B.25) ;

- La commémoration d'une construction par (la tribu de) Ma`īn et le Haḍramawt (M 423).

Avec le royaume de Qatabān :

- La présence d'un chef $(k b r)$ des minéens dans la capitale Tamna et la réalisation d'une tombe pour les Minéens de Tamna' (Van Lessen $9=$ CSAI I, 72) ;

- La dédicace conjointe de la restauration de l'enceinte de Yathill « aux jours de leur seigneur $W q h^{\prime} l Y \underline{t}^{c}$ et de son fils 'lyf $Y s^{2} r$ rois de Ma'īn, avec son seigneur $S^{2} h r Y g l$ Yhrgb roi de Qatabān » (RÉS 2999);

- La dédicace d'un lion de bronze par une Minéenne dans un sanctuaire qatabānite, aux dieux et aux rois de $\mathrm{Ma}^{\mathrm{i}} \mathrm{i} n$ et Qatabān.

- On note enfin que neuf des sous-clans minéens attestés à Barāqish ont pour homonyme un lignage ou patronyme sur les sites qatabānites (avec parfois l'ajout de mīmation), principalement dans la ville de Tamna' et sa nécropole Hayd Ibn 'Aqīl (tableau 2). La fréquence du phénomène ne semble pas être le fruit du hasard et on peut postuler dans certains cas une origine minéenne ou le résultat de mariages mixtes. 


\begin{tabular}{|c|c|c|}
\hline $\begin{array}{l}\text { Sous-clan attesté } \\
\text { dans les inscriptions } \\
\text { de Barāqish }\end{array}$ & $\begin{array}{l}\text { Lignage / patronyme } \\
\text { attesté dans les } \\
\text { inscriptions qatabānites }\end{array}$ & Inscription qatabānite \\
\hline Dfgn & $D f g n$ & ATM 694 \\
\hline Dmrn & Dmrn & $\begin{array}{l}\text { BM 141583, CSAI I, 208, 304, 306, } \\
313,579,660,665,968\end{array}$ \\
\hline Hbrr & Hbrrm & CSAI I, 144, 148 \\
\hline Mlh & Mlḥm & CSAI I, 208, 669, 971, MuB 144 \\
\hline$M r n$ & $M r n$ & CSAI I, 53, 96, 208 \\
\hline Rwyn & Rwyn & MuB 694, ATM 610 \\
\hline $\begin{array}{lll}S^{2 c} \underline{t} m & \text { (fraction } \\
\left.G b^{2} n\right) & \end{array}$ & $S^{2^{c}} \underline{t} m m$ & CIH 947, CSAI I, 155, 208, 1054, 1069 \\
\hline$Y f^{\prime} n$ (fraction de $G b^{\prime} n$ ) & $Y f^{c} n$ & TC 1996 \\
\hline $\begin{array}{l}\text { Mhạdr (fraction de } \\
\text { Ylqz) }\end{array}$ & Mḥdrm & $\begin{array}{l}\text { ATM } 866, C S A I \text { I, } 47,208,269,562, \\
563,600,962\end{array}$ \\
\hline
\end{tabular}

Tableau 2 : Liste des sous-clans de Barāqish et leurs homonymes qatabānites.

A Yathill, la richesse qu'ont procurée les recettes commerciales furent déterminantes dans la monumentalisation de la ville. Une dîme sur les recettes commerciales était en effet prélevée par les temples. La construction monumentale était une manière de s'acquitter de cette taxe due au temple de 'Athtar dhu-Qabḍum (RÉS 2975, RÉS 3012, RÉS 3021, RÉS 3022, RÉS 3535).

L'activité architecturale et caravanière atteignent un pic aux $5^{\mathrm{e}}-4^{\mathrm{e}}$ siècles av. J.-C. Les siècles suivants sont moins bien renseignés. Il n'est pas improbable que la concurrence des royaumes de Muha'mir puis Amīr dans le wādī Najrān, de la cité de Qaryat al-Fāw, de celle de Hagar/Gerrha en Arabie orientale et de Maryamat ${ }^{u m}$ dans le wādī Harāīb se soit accrue, freinant progressivement l'essor du royaume de Ma'īn.

\subsection{Yathill, déclin et abandon ( $1^{\mathrm{er}}-3^{\mathrm{e}}$ siècle ap. J.-C. $)$}

Au cours du $1^{\text {er }}$ siècle av. J.-C., les derniers investissements monumentaux sont attestés à Yathill : le rempart est restauré vers -70 (RÉS 2973, règne de $\left.Y \underline{t}^{\prime \prime} l S d q\right)$; il est fait l'acquisition

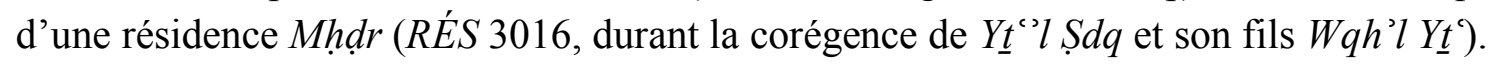

Les habitants de Yathill n'opposent qu'une faible résistance face à l'expédition romaine menée par Ælius Gallus (-26/-25). Strabon mentionne le passage de l'armée romaine par la ville d' 'A $\theta \rho o v \lambda \alpha$, qui se rend sans coup férir. L'armée romaine s'y réapprovisionne en blé et en dattes (Strabon Géogr., 16, 4, 24). C'est probablement à cette occasion là qu'est gravée la pierre tombale gréco-latine du cavalier Publius Cornelius provenant de Barāqish (YM 605) ${ }^{13}$.

A la suite du passage de l'armée romaine, les derniers travaux de réfection connus sur le rempart sont entrepris sous les règnes conjoints de $W q h^{\prime} l Y \underline{t}^{c}$ et ' $l y f^{c} Y s^{2} r$ rois de Ma īn et $S^{2} h r Y g l Y h r g b$ roi de Qatabān (RÉS 2999) sans que l'on puisse affirmer si ceux-ci sont la conséquence de destructions engendrées par l'armée romaine.

\footnotetext{
${ }^{13}$ Une abondante bibliographie est consacrée à cette inscription bilingue. Sur les interprétations et sa datation controversée, voir Arbach \& Schiettecatte 2017 : 684-85 et références citées.
} 
Au tournant de l'ère chrétienne, les indices d'une occupation du site sont limités et soulignent l'arrivée de nouvelles populations issues des tribus de Amīr et de Saba'.

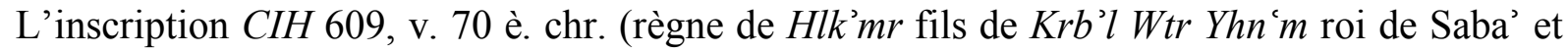
dhu-Raydān), montre qu'en moins d'un siècle, Ma'īn est passé du statut de royaume indépendant à celui de tribu dépendant de Saba’ (Robin 1998 : 184). Ce texte est un acte juridique concernant la cession de propriétés foncières (palmeraies et terres agricoles) et immobilières dans les villes de Qarnā, Yathill et Shu ub. Yathill y apparaît encore comme une ville peuplée et entourée d'un secteur irrigué ; la tribu de Yathill n'est en revanche pas nommée. Vers 90 è. chr., le territoire de Yathill est au cœur des affrontements qui opposent l'armée de $Y d^{(\prime} l$ roi du Haḍramawt à celle de Krb'l Byn roi de Saba' et dhu-Raydān (Ja 643). L'armée du Haḍramawt y trouve refuge avant d'en être chassée.

Ce même événement semble avoir été à l'origine de l'acte de confession des tribus de Amīr et 'Athtar à Halfān, sur le site de Haram, qui n'avaient pu s'acquitter de la chasse rituelle de Ḥalfān et avaient dû trouver refuge à Yathill « durant la guerre de Haḍramawt» (Haram 10). Les membres de ces deux tribus mentionnent avoir alors effectué le pèlerinage de dhu-Samāwī à Yathill. Cet événement pourrait trouver un écho à Barāqish dans la présence de l'inscription Y.92.B.A 20 commémorant l'offrande de deux statues à dhū-Samāwī dans le temple de Nakrah (voir 5.2.2 - Byn, temple de dhū-Samāwī à Barāqish (?)) et d'une dédicace au dieu Halfān (Y.05.B.B.16) dans le temple de 'Athtar dhu-Qabḍ̂m (voir 5.2.3 - $Q b d^{m}$, temple de 'Athtar à Barāqish).

Ces textes illsutrent la réappropriation/réutilisation des temples de Yathill par les tribus d'Amīr et 'Athtar pour le culte de leurs divinités, dhu-Samāwī et Halfān. Il n'est pas possible de préciser si ces tribus ont trouvé refuge à Yathill de manière ponctuelle ou si elles s'y sont établies durablement. Ces tribus, originaire de la région de Najrān, étaient déjà présentes dans la ville voisine de Haram dès le $2^{\mathrm{e}}$ siècle av. J.-C. (Robin 1991).

Dans la seconde moitié du $3^{\mathrm{e}}$ siècle è. chr., la ville de Yathill est mentionnée pour la dernière fois dans la documentation épigraphique comme le lieu à proximité duquel un représentant du roi de Saba’ à Nashq (actuelle al-Bayḍā'), avait fait une lourde chute de dromadaire. À cette période, Yathill n'est mentionnée ni dans la liste des villes et des tribus fournissant des contingents militaires au roi ni comme lieu d'origine de dédicants dans le temple confédéral de Ma’rib (Robin 1987 : 165). Elle ne semble plus occupée que sporadiquement, par des groupes de taille limitée, n'engageant aucun chantier monumental, à l'image de ce qu'ont révélé les fouilles archéologiques (Fedele 2010 : 138 ; Agostini 2015 : 11).

Malgré leur abandon, les remparts étaient suffisamment bien préservés pour qu'au $10^{\mathrm{e}}$ siècle, al-Hamdānī mentionne le site, alors connu sous son nom actuel, Barāqish, comme citadelle ( $m h f d$ - al-Hamdānī, al-Iklīl $8: 65$ ). Il fut réoccupé deux siècles plus tard et de manière quasicontinue jusqu'au $18^{\mathrm{e}}$ siècle.

\section{Les monuments de Barāqish}

Le corpus des inscriptions de Barāqish et des sites environnants nous renseigne inégalement sur l'architecture et l'activité de construction. La très grande majorité des textes date d'une période comprise entre le $5^{\mathrm{e}}$ et le $1^{\mathrm{er}}$ siècle av. J.-C. Très peu d'éléments nous sont connus des premiers et derniers siècles de l'occupation du site. Par ailleurs, les textes apportent des 
éclairages sur le rempart et les temples. Les constructions hydrauliques et domestiques sont mentionnées de manière plus occasionnelle. C'est là un artifice lié à l'histoire de l'élaboration du corpus épigraphique, qui s'est avant tout constitué à travers l'étude du rempart d'une part et des temples intra et extra muros d'autre part. L'annexe 2 propose un tableau récapitulatif de l'ensemble des constructions connues par les textes.

\section{$5.1 \quad$ Le rempart}

\subsubsection{Les éléments du rempart}

Le rempart tel qu'il est actuellement visible se compose de 57 bastions ${ }^{14}$ - également appelés tours ou saillants dans la littérature spécialisée - et de courtines en nombre presque identique. Ce rempart est une restauration médiévale du rempart antique, dont il reprend le tracé. En élévation, il est rare que le rempart antique soit préservé au-delà de quelques assises. Seul le bastion 48 est intégralement conservé. On entendra par rempart antique le rempart tel qu'il était au moment de l'abandon du site vers les $2^{\mathrm{e}}-3^{\mathrm{e}}$ siècles è. chr., il s'agit donc du rempart bâti à l'époque minéenne (voir « 5.1.2 - Essai de mise en ordre chronologique »).

Chacun de ces bastions et courtines était baptisé d'un nom propre dont les inscriptions ont parfois gardé le souvenir. Certaines dédicaces de construction sont encore visibles, tantôt dans leur emplacement d'origine, en partie inférieure, tantôt remployées dans les assises supérieures du mur. Associer les noms des bastions et courtines connus par les textes avec une structure architecturale permet de retracer l'histoire de la construction du rempart. Cette entreprise se confronte à une difficulté : lorsque le nom est connu par un texte remployé, il n'est jamais assuré que celui-ci ait été remployé dans la construction d'origine. On peut néanmoins faire l'hypothèse d'une mobilité limitée des inscriptions lors de leur remploi pour deux raisons. Premièrement, lorsqu'une construction est identifiée à la fois par un texte en place et par un texte remployé, ce dernier est soit remployé dans la construction même soit à proximité immédiate. C'est le cas des bastions $L b^{\prime} n$ (I), $L b^{\prime} n$ (II), Nmrn et de la courtine $T s^{2} b m$ (I), dans une moindre mesure de la courtine $T n^{\prime} m$ (mentionnée dans un remploi quatre bastions plus loin). Deuxièmement, on observe que les fragments d'une même inscription ne sont jamais remployés loin les uns des autres. Sur treize cas observés, ils sont soit remployés dans un même bastion $^{15}$, soit dans un bastion et son voisin ${ }^{16}$, soit au maximum à deux bastions de distance ${ }^{17}$. C'est là encore la preuve d'une dispersion moindre.

\footnotetext{
${ }^{14}$ Nous utilisons ici la numérotation des bastions proposée par la MAIRY, qui commence dans l'angle sud-ouest du rempart et suit le tracé du rempart dans le sens des aiguilles d'une montre. Cette numérotation est différente de celle qui a été proposée et utilisée par la MAFRAY (Mission Archéologique Française en République Arabe du Yémen), qui débutait dans le même secteur et suivait le rempart dans le sens inverse des aiguilles d'une montre — se reporter notamment à Robin 1979a : fig. 1 et à Breton 1994 : fig. 42 qui amende le plan de Ch. Robin par le décalage du bastion numéro 1 et l'ajoute d'un numéro 58 .

${ }^{15}$ B-M $111=$ M $431+432$ dans le bastion 35 ; B-M $139=R E ́ S 2991+2993+2994$ dans le bastion 38 ; B-M 253 $=$ M $405+189$ dans le bastion $52 ; \mathrm{B}-\mathrm{M} 256=$ RÉS $2972+2970+2971+2971$ bis B +2971 bis A dans le bastion 54

${ }^{16} \mathrm{~B}-\mathrm{M} 26+23$ dans les bastions 5 et 6 ; B-M $44=R E ́ S 3040+3039+3049$ dans les bastions 15 et 16 ; B-M 73 $=R E S S 3053+3056+3050$ et $R E ́ S 3051+3052 \mathrm{a}$ dans les bastions 23 et $24 ; \mathrm{B}-\mathrm{M} 115=\mathrm{M} 436+430$ dans les bastions 36 et 37 ; B-M $132=$ M $439+212$ dans les bastions 37 et 38 ; B-M $182=R E ́ S 2929+2941+2945+$ 2946 dans les bastions et courtines 41 et 42 .

${ }^{17} \mathrm{~B}-\mathrm{M} 38=R E ́ S 3035+3048$ dans les bastions 14 et 16 ; B-M $136=R E ́ S 2996+2995+2988+2992$ dans les bastions et courtines 37,38 et 39 ; B-M $247+255=R E ́ S 2966+2968$ dans les bastions 50 et 52
} 
Vingt bastions et quatorze courtines sont nommés par des inscriptions. Une majorité peut être placée avec plus ou moins d'assurance sur le plan (fig. 1).

\subsubsection{1 [.] $r^{3} n$}

Bastion $(m h f d)$ mentionné dans l'inscription de fondation B-M 272 = RÉS 3021. Sa construction (ou reconstruction) est commanditée par des membres du sous-clan $S^{2} \underline{t} m$, dont l'inscription Ma'īn 95/10-11 nous apprend qu'il s'agit d'une fraction du clan Gb'n. Ces travaux interviennent sous le règne de ' $l y f^{c} Y s^{2} r$ (I) et de son fils $H f n^{m} R y m$, vers les $3^{\mathrm{e}}-2^{\mathrm{e}}$ siècles av. J.C. ${ }^{18}$ Le bastion [.] $r^{3} n$ est mentionné dans une inscription conservée en place dans le bastion 57, au sein d'une énumération de sept bastions dont trois sont localisés (1=Rbqn (I) ; $54=L b^{\prime} n$ (I) ; $55=Z r b n$ ). On peut faire l'hypothèse que les quatre bastions non localisés se trouvent dans le même secteur, a priori les bastions $n^{\circ} 50$ à 53 , les seuls qui restent non identifiés dans la section comprise entre le bastion $n^{\circ} 43$ à l'est et $n^{\circ} 2$ à l'ouest. On ne peut pas non plus exclure qu'il s'agisse d'un ensemble situé à l'ouest du site (nº 3 à 6 par exemple).

\subsubsection{2 'brm}

Courtine (?) ([sh]ftn) mentionnée dans l'inscription B-M 108. L'auteur de l'inscription se dit serviteur de $W q h^{\prime} l$. Il s'agit de l'un des cinq rois de Ma'īn portant ce nom. D'après la graphie, le candidat le plus vraisemblable serait $W q h^{\prime} l ~ N b t$ (v. $3^{\mathrm{e}}-2^{\mathrm{e}}$ siècles av. J.-C.) mentionné dans BM $1=R E ́ S 2975, \mathrm{~B}-\mathrm{M} 136=R E ́ S 2996+2995+2988+2992$ et $R E ́ S 3707$.

\subsubsection{3 $\underline{d}$-Bqrn}

Bastion (mhfd) mentionné dans l'inscription B-M $241=R E S 2965$ dans le cadre de l'aménagement de ses parements $(t z w r t)$. Cet aménagement est commandité par des membres du sous-clan $H f d$, fraction du clan $G b^{\prime} n$. Ces travaux interviennent sous le règne de ' $b k r b S d q$, vers le $4^{\mathrm{e}}$ siècle av. J.-C. L'inscription est conservée en place, sur le bastion 48 . Nous faisons l'hypothèse que le bastion $\underline{d}$-Bqrn dont elle mentionne la construction lui correspond. Cette localisation a déjà été reconnue par Ch. Robin et A. Agostini (Robin 1979a : fig. 3 ; Agostini 2011 : fig. 5).

\subsubsection{4 d-Hfn}

Bastion $(m h f d)$ mentionné dans l'inscription de fondation B-M $182=R E ́ S 2929+2941+2945$ +2946 . Sa (re)construction est commanditée par des membres du sous-clan $H f n$, fraction du clan $G b^{\prime} n$. Ces travaux interviennent sous le règne de 'byd $Y \underline{t}^{c}$ et de son fils $W q h^{\prime} l R y m$, vers les $5^{\mathrm{e}}-4^{\mathrm{e}}$ siècles av. J.-C. B-M 182 nous apprend que le bastion $\underline{d}-H f n$ est relié au bastion $L b^{\prime} n$ par une courtine. Il existait deux bastions portant le nom $L b^{\prime} n$ : l'un identifié avec le bastion 54 ou 55 et l' autre avec le bastion 44 (voir $L b^{\prime} n$ (I) et $L b^{\prime} n$ (II) au-dessous). B-M 182 est remployée par fragments dans les bastions 41 et 42 et la courtine qui les sépare. La proximité avec $L b^{\prime} n$ (II) (bastion 44) fait supposer que le bastion $\underline{d}-H f n$ était connecté à ce dernier. Il s'agirait donc du bastion 43. Cette localisation diffère de celle proposée par Ch. Robin (1979a : fig. 4) et A. Agostini (2011 : fig. 5) qui localisent $L b^{\prime} n$ (II) et $\underline{d}-H f n$ au niveau des bastions 41 et 42 , lieu de leur remploi. L'hypothèse qui nous conduit à privilégier les bastions 43 et 44 est développée sous l'entrée $L b^{\prime} n$ (II).

Le bastion $\underline{d}-H f n$ est un des rares exemple de construction portant le nom du sous-clan de ses bâtisseurs.

\footnotetext{
${ }^{18}$ Concernant l'ordre et la date des règnes, voir l'Annexe 4.
} 


\subsubsection{5 d-Hs $\underline{d} b r$}

Courtine (șft) mentionnée dans l'inscription de fondation B-M $272=R E ́ S$ 3021. Sa (re)construction est commanditée par des membres du sous-clan $S^{2} \underline{t} \underline{m}$, dont l'inscription Ma ${ }^{c} \bar{i} n$ 95/10-11 nous apprend qu'il s'agit d'une fraction du clan $G b^{\prime} n$. Ces travaux interviennent sous le règne de ' $l y f^{c} Y s^{2} r$ (I) et de son fils $H f n^{m} R y m$, vers les $3^{\mathrm{e}}-2^{\mathrm{e}}$ siècles av. J.-C. Il s'agit de l'une des courtines non localisées que liste $R E ́ S 3021$ dans le secteur sud-ouest de la ville.

\subsubsection{6 d-Mlh}

Bastion $(m h f d)$ mentionné dans l'inscription de fondation B-M $10=R E ́ S$ 3012. Sa (re)construction est commanditée par des membres du sous-clan $Y f^{\prime} n$, dont l'inscription Ma ${ }^{c} \bar{i} n$ $93 \mathrm{C} / 52$ nous apprend qu'il s'agit d'une fraction du clan $G b^{\prime} n$. Ces travaux interviennent sous le règne de 'byd $d^{c} Y \underline{t}^{c}$ et de son fils $W q h^{\prime} l$ Rym, vers les $5^{\mathrm{e}}-4^{\mathrm{e}}$ siècles av. J.-C. L'inscription B-M 10 est conservée à sa place d'origine, à la base du bastion. L'identification de $\underline{d}$-Mlh avec le bastion 2 est assurée. Cette localisation avait déjà été reconnue par Ch. Robin (1979a : fig. 2) et J.-F. Breton (1994 : 110-11).

L'inscription B-M 1 = RÉS 2975 mentionne l'aménagement d'un passage maçonné entre le bastion $\underline{d}-M l h$ (bastion 2) et la tour Rbqn (tour 1) lors de l'édification de celle-ci, sous le règne postérieur de ' $l y f^{c} Y s^{2} r$ et de son fils $W q h^{\prime} l N b t$ ( $\left(3^{\mathrm{e}}-2^{\mathrm{e}}\right.$ siècles av. J.-C.).

Le bastion $\underline{d}$-Mlh porte le nom d'une fraction de clan minéen (YM 28336, Qaryat-Zaydwadd, $R E ́ S$ 3272). Il ne s'agit pas cette fois du sous-clan des commanditaires, tel qu'on a pu l'observer avec le bastion $\underline{d}-H f f n$. Il n'est pas certain dans le cas présent qu'il y ait un lien entre le nom du bastion et celui du clan.

Notons néanmoins que l'on retrouve également le nom Mlh de manière isolée sur plusieurs bastions et courtines de la partie nord du rempart (fig. 1 : bastions 11, 13, 20, 21, 23, courtines 11-12, 15-16, 17-18, 18-19). Là encore, le lien entre ce nom, le rempart et celui du sous-clan minéen ne peut pas être clairement établi.

\subsubsection{7 $\underline{d}-\mathrm{Ndbn}$}

Bastion $(m h f d)$ mentionné dans trois inscriptions qui semblent se rapporter à la même structure. L'inscription de fondation Y.03.B.R44-45.2bis + 2ter +2 évoque la construction de ce bastion et de la courtine $T f s^{2}$ sous le règne de 'byd' $Y \underline{t}^{c}$ et de son fils $H y w^{m}$, vers le $5^{\mathrm{e}}$ siècle av. J.-C. Les commanditaires ne sont pas identifiés. Toutefois, l'inscription Y.92.B.A 37 A-B, qui se rapporte aussi à la fondation du bastion $\underline{d}-N d b n$ et de la courtine $T f s^{2}$, a pour auteurs des membres du sous-clan $\dot{G} r b t$, dont l'inscription Ma'in 93B/30 nous apprend qu'il s'agit d'une fraction du clan $M w q h$. C'est la seule attestation de la construction d'un élément du rempart par un autre clan que celui de $G b^{\prime} n$.

Notons que quelques générations plus tard, sous le règne de ' $b k r b S d q$, des parements $(t z w r)$ sont maçonnés au niveau de cette tour par le sous-clan $H f d$, fraction de $G b^{\prime} n$ (B-M $241=R E ́ S$ 2965).

Les deux courtines qui encadrent le bastion $d-N d b n$ sont connues : $S^{l} l f$ ('lhn) d'une part (B-M $241=R E ́ S$ 2965) ; Tf $S^{2}$ d'autre part (Y.92.B.A 37 A-B ; Y.03.B.R44-45.2bis +2 ter + 2).

Parmi les trois inscriptions qui mentionnent la construction du bastion $\underline{d}-N d b n$, l'une (Y.03.B.R44-45.2bis +2 ter +2 ) a été trouvée en fouilles entre les saillants $44-45^{19}$ et les fouilleurs font l'hypothèse de la proximité de l'inscription avec les structures qu'elle décrit

\footnotetext{
${ }^{19}$ Voir la contribution d'Alessio Agostini dans ce volume.
} 
(bastion $\underline{d}-N d b n$ et courtine $T f s^{2}$ ). La tour $\underline{d}-N d b n$ pourrait correspondre au bastion 45 et serait bordée par les courtines $S^{1} l f$ à l'ouest et $T f s^{2}$ à l'est (sur le choix de placer $T f s^{2}$ à l'est, se reporter à l'entrée concernée). Une identification de $\underline{d}-N d b n$ avec le bastion 44 est moins probable (voir l'entrée $L b^{\prime} n$ (II), localisé à cet emplacement). La seconde inscription (Y.92.B.A 37 A-B), provient du temple de Nakrah, à l'arrière des bastions 44-45, et conforte l'identification proposée. La troisième inscription (B-M $241=R E ́ S$ 2965) est conservée in situ sur le bastion 48 (bastion $\underline{d}$-Bqrn). La proximité relative avec le bastion 45 ne contredit pas l'identification de ce dernier.

\subsubsection{8 $\underline{d}-S^{2} f t n$}

Courtine (ṣft) mentionnée dans l'inscription B-M $241=R E ́ S 2965$ dans le cadre de la réalisation de son parement $(t z w r)$. Cet aménagement est commandité par des membres du sousclan $H f d$, fraction du clan $G b^{\prime} n$. Ces travaux interviennent sous le règne de 'bkrb $S d q$, vers le $4^{\mathrm{e}}$ siècle av. J.-C. La courtine est mentionnée dans le secteur du saillant 48 sans que sa position précise ne puisse être localisée. Nous faisons l'hypothèse d'une courtine attenante au saillant 48.

\subsubsection{Ddn}

Courtine $(s h f t)$ mentionnée dans l'inscription de fondation B-M $56=R E ́ S$ 3060. Sa (re)construction est commanditée par des membres du sous-clan $Y f^{\prime} n$, dont l'inscription Ma īn $93 \mathrm{C} / 52$ nous apprend qu'il s'agit d'une fraction du clan $G b^{\prime} n$. Ces travaux interviennent sous le règne de 'byd $R y m$, vers le $4^{\mathrm{e}}$ siècle av. J.-C. L'inscription est conservée à son emplacement d'origine et permet d'identifier $D d n$ avec la courtine comprise entre les bastions 18 et 19. Cette localisation avait déjà été reconnue par Ch. Robin (1979a : fig. 5).

Ce bastion tourné dans la direction de la lointaine Dadān (oasis d'al-`Ulā), pourrait tenir son nom de ce partenaire commercial.

\subsubsection{0 $\mathrm{Hrs}^{2}$}

Courtine (shft) mentionnée dans l'inscription B-M $272=R E ́ S$ 3021. Sa (re)construction est commanditée par des membres du sous-clan $S^{2} \underline{t}$ tm, dont l'inscription Ma ${ }^{\top} \bar{n}$ 95/10-11 nous apprend qu'il s'agit d'une fraction du clan $G b^{\prime} n$. Ces travaux interviennent sous le règne de ' $l y f^{\mathrm{c}} Y s^{2} r$ (I) et de son fils $H f n^{m} R y m$, vers les $3^{\mathrm{e}}-2^{\mathrm{e}}$ siècles av. J.-C. Il s'agit de l'une des courtines non localisées que liste $R E ́ S 3021$ dans le secteur sud-ouest de la ville.

\subsubsection{Lb'n (I) (Lb’n $\left.\underline{d}-{ }^{c} n n\right)$}

Bastion $(m h f d)$ dont l'inscription de fondation, B-M 256 $=$ RÉS $2972+2970+2971+2971$ bis, rapporte qu'il fut commandité par des membres du sous-clan $B l h$, fraction du clan $G b^{\prime} n$, sous le règne de 'byd $Y \underline{t}^{\mathrm{c}}$, vers le $5^{\mathrm{e}}$ siècle av. J.-C. Parmi ces membres se trouve un certain ' $m$ 'ns $s^{1}$. L'inscription Y.92.B.A $21+30$, datée du même règne, est rédigée par ' $m s^{l} m^{\text {' }} \underline{d}-B l h$, kabīr de Yathill, attesté dans plusieurs inscriptions. Ce second texte évoque la tour $L b^{\prime} n \underline{d}-{ }^{\prime} n n$. Nous pourrions avoir ici le nom complet du bastion, dont la construction aurait été entreprise par le père et se serait poursuivie sous la conduite du fils.

Une phase de restauration de plusieurs bastions et courtines entreprise par des membres du sous-clan $S^{2 c} \underline{t} m$, fraction du clan $G b^{\prime} n$ est entreprise sous le règne postérieur de ' $l y f^{c} Y s^{2} r$ (I) et de son fils $H f n^{m}$ Rym (B-M $\left.272=R E ́ S 3021\right)$.

D'après $R E S S 3022$, les bastions $L b^{\prime} n$ et $Z r b n$ constituent les extrémités de la courtine $T n^{\text {‘ }} m$, que l'on peut précisément localiser entre les bastions 54 et 55 (voir l'entrée correspondante). 
L'inscription de fondation du bastion $L b^{\prime} n$, B-M 256, étant remployée dans le bastion 54, celuici apparaît comme le meilleur des deux candidats. Dans ce cas, le bastion 55 serait Zrbn. Ces localisations avaient déjà été envisagée (Robin 1979a : fig. 2). Elles diffèrent des restitutions de J.-F. Breton (1994: 110-11) et d'A. Agostini (2011 : fig. 5) qui identifient $L b^{\prime} n$ avec le bastion 55 et $Z r b n$ avec le 54 .

\subsubsection{Lb'n (II)}

Bastion ( $m h f d$ ) homonyme mais distinct du précédent dont l'inscription de fondation (B-M 182 $=R E S 2929+2941+2945+2946)$ date de la corégence de 'byd I $^{c}$ ' et de son fils $W q h^{\prime} l$ Rym, vers les $5^{\mathrm{e}}-4^{\mathrm{e}}$ siècles av. J.-C. Ses commanditaires sont membres du sous-clan $H f f$, fraction du clan $G b^{\prime} n$. Un second texte commémore la construction de ce bastion par les membres d'un même clan, sous le même règne (B-M 211 + B-M $204=R E ́ S 2952$ + 2949).

B-M 182 mentionne qu'un bastion $\underline{d}-H f n$ était relié au bastion $L b^{\prime} n$ par une courtine. Le remploi de ce texte par fragments entre les bastions 41 et 42 amène Ch. Robin (1979a : fig. 4) et $\mathrm{A}$. Agostini (2011 : fig. 5) à localiser les bastions $\underline{d}-H f n$ et $L b^{\prime} n$ aux $n^{\circ} 41-42$.

Néanmoins, l'inscription B-M 211 + B-M 204 se trouve remployée entre les bastions 43 et 44 et rapporte que le bastion $L b^{\prime} n$ fait face à la porte de $Y \underline{t} l$. Le bastion 44 fait face à la poterne qui donne accès au temple de Nakrah. Il apparaît donc comme la meilleure hypothèse d'identification de $L b^{\prime} n$ (II).

\subsubsection{3 $M \underline{d} b$}

Courtine (șft) mentionnée dans l'inscription de fondation B-M $238=R E ́ S 3535$ (fragment de Bauer 5). Sa (re)construction est commanditée par des membres du sous-clan $D f g n$, fraction d'un clan qui ne peut être identifié en l'état. Ces travaux interviennent sous le règne de 'byd' $Y \underline{t}^{c}$ et de son fils $W q h^{\prime} l$ Rym, vers les $5^{\mathrm{e}}-4^{\mathrm{e}}$ siècles av. J.-C.

L'inscription de fondation est conservée dans son emplacement d'origine, entre les bastions 47 et 48. Cette localisation a déjà été reconnue par Ch. Robin, J.-F. Breton et A. Agostini (Robin 1979a : fig. 3 ; Breton 1994 : 111 ; Agostini 2011 : fig. 5).

\subsubsection{Nmrn}

Bastion $(m h f d)$ mentionné dans l'inscription de fondation B-M $6=R E ́ S$ 3025. Son commanditaire est un souverain de Ma`īn dont le nom n'est pas préservé. Il s'agit de l'unique intervention directe d'un roi de Ma 'īn sur le rempart de Barāqish.

L'inscription de fondation est remployée dans la tour 1 (Rbqn) et ne nous renseigne guère sur la localisation. Une seconde inscription commémore sa construction (B-M $264=R E ́ S 3015)$ et décrit le bastion $N m r n$ comme attenant à la courtine $T s^{2}[\mathrm{bm}]$, à proximité de la porte de la ville. Si la localisation proposée pour $T s^{2} b m$ est correcte, à savoir la courtine percée par la porte de la ville entre les bastions 56 et 57 (voir sous « $T s^{2} b m »$ ), Nmrn serait le bastion 56. C'est d'une part celui dans lequel l'inscription B-M 264 est remployée, d'autre part le bastion 57 ne peut être retenu car il semble assuré qu'il ait porté le nom de $Y \underline{t}{ }^{c} n$ (voir l'entrée correspondante).

\subsubsection{Rbqn (I)}

Tour $(m h f d)$ située à l'extrémité sud-ouest du rempart de Barāqish. Elle est reliée au bastion $\underline{d}$ Mlh ( $\mathrm{n}^{\circ}$ 2) par un passage maçonné. Cette tour est un ajout postérieur au rempart. Sa construction est commémorée par une inscription de fondation, B-M $1=R E ́ S 2975$, datée de la corégence de ' $l y f^{\mathrm{t}} Y s^{2} r(\mathrm{I})$ et $\left[W q h^{ } l\right] N b t$, vers les $3^{\mathrm{e}}-2^{\mathrm{e}}$ siècles av. J.-C. L'inscription, préservée 
à son emplacement d'origine, nous donne l'emplacement de Rbqn (tour $\mathrm{n}^{\circ} 1$ ). Cette localisation a déjà été reconnue par Ch. Robin (1979a : fig. 2) et J.-F. Breton (1994 : 110-11).

Les commanditaires des travaux sont membres du sous-clan Zlwmn, fraction du clan $G b^{\prime} n$.

La construction de la tour Rbqn a vraisemblablement débuté dès la corégence qui précède, entre 'lyf $Y s^{2} r$ (I) et son fils $H f n^{m} R y m$ (voir Annexe 4) car son nom apparaît dans B-M $272=R E S$ 3021, qui liste les tours et bastions édifiés dans le secteur sud-ouest de la ville.

\subsubsection{Rbqn (II)}

Bastion $(m h f d)$ homonyme mais distinct du précédent également mentionné dans l'inscription B-M $272=R E ́ S$ 3021. Sa (re)construction est commanditée par des membres du sous-clan $S^{2 c} \underline{t} m$, fraction du clan $G b^{\prime} n$ (Ma'in 95/10-11). Ces travaux interviennent sous le règne de 'lyf $Y s^{2} r$ (I) et de son fils $H f n^{m} R y m$, vers les $3^{\mathrm{e}}-2^{\mathrm{e}}$ siècles av. J.-C. Le bastion Rbqn (II) est mentionné dans une inscription conservée en place dans le bastion 57, au sein d'une énumération de sept bastions dont trois sont localisés $\left(1=R b q n\right.$ (I) ; $54=L b^{\prime} n$ (I) ; $\left.55=Z r b n\right)$. On peut faire l'hypothèse que les quatre bastions non localisés se trouvent dans le même secteur, a priori les bastions $n^{\circ} 50$ à 53 , les seuls qui restent non identifiés dans la section comprise entre le bastion $\mathrm{n}^{\circ} 43$ à l'est et $\mathrm{n}^{\circ} 2$ à l'ouest. On ne peut pas non plus exclure qu'il s'agisse d'un ensemble situé à l'ouest du site ( $n^{\circ} 3$ à 6 par exemple).

\subsubsection{Rdwn}

La description de Rbqn (II) s'applique en tout point à $R d w n$. Il s'agit de l'un des bastions non localisés que liste RÉS 3021 dans le secteur sud-ouest de la ville, peut-être l'un de ceux que nous ne sommes pas parvenus à identifier (bastions n 50 à 53 ).

\subsubsection{Rt}

Courtine (shft) mentionnée dans l'inscription B-M $266=R E ́ S$ 4224. Cette inscription fragmentaire évoque des travaux commandités à une date indéterminée par le sous-clan $Y f^{c} n$, fraction du clan $G b^{\prime} n$ (d'après Ma īn $93 \mathrm{C} / 52$ ). Cette courtine n'est pas localisée. L'inscription B-M 266 est remployée dans la courtine qui sépare les bastions 56 et 57 ; par conséquent, la courtine se trouvait vraisemblablement dans le secteur sud-ouest de la ville.

\subsubsection{Silf}

Courtine (shft) mentionnée dans l'inscription B-M 241 = RÉS 2965 lors de la réalisation de son parement $(t z w r)$. Cet aménagement est commandité par des membres du sous-clan $H f d$, fraction du clan $G b^{\prime} n$, sous le règne de ' $b k r b S d q$, vers les $4^{\text {e }}$ siècle av. J.-C. D'après B-M 241, la courtine jouxtait le bastion $\underline{d}-N d b n$, identifié au bastion $n^{\circ} 45$, à l'est ou à l'ouest de ce dernier.

\subsubsection{0 $S^{2} b m t$}

Construction mentionnée dans l'inscription de fondation B-M $10=R E ́ S$ 3012, dont la construction est commanditée par des membres du sous-clan $Y f^{*} n$, fraction du clan $G b^{\prime} n$ (d'après Ma ${ }^{\text {īn }} 93 \mathrm{C} / 52$ ). Ces travaux interviennent sous le règne de 'byd $Y \underline{t}^{c}$ et de son fils Wqh'l Rym, vers les $5^{\mathrm{e}}-4^{\mathrm{e}}$ siècles av. J.-C. Bien que trouvée dans son emplacement d'origine, $R E ́ S 3012$ comporte des lacunes et la nature de la structure $S^{2} b m t$ n'est pas connue. Toutefois, elle est mentionnée conjointement à $T s^{2} b m$, une courtine localisée non loin du lieu de l'inscription B-M 10. $S^{2} b m t$ était vraisemblablement elle aussi une courtine localisée dans le secteur sud-ouest du rempart. 


\subsubsection{Sdqn}

Bastion $(m h f d)$ mentionné dans l'inscription Y.05.B.B.13, dont la construction est commanditée par des membres du sous-clan $\dot{G} z r-S^{\prime} h f n$, fraction d'un clan non identifié. Ces travaux interviennent sous le règne de $W q h^{\prime} l$ Rym, vers la fin $5^{\mathrm{e}}$-début $4^{\mathrm{e}}$ siècle av. J.-C. L'inscription provient du temple de 'Athtar dhū-Qabḍ, non du rempart lui-même et le bastion ne peut pas être localisé.

\subsubsection{TfS ${ }^{2}$}

Courtine (shft) dont la construction est commémorée par deux inscriptions, Y.03.B.R44-45.2bis +2 ter +2 et Y.92.B.A 37 A-B. La première est datée du règne de 'byd ${ }^{c} \underline{t}^{c}$ et de son fils $H y w^{m}$, vers le $5^{\mathrm{e}}$ siècle av. J.-C. ; la seconde, non datée, a pour auteurs des membres du sous-clan $\dot{G} r b t$, dont l'inscription Ma'īn 93B/30 nous apprend qu'il s'agit d'une fraction du clan $M w q h$. Les deux textes en font une courtine attenante au bastion $\underline{d}-N d b n$, identifié au bastion $n^{\circ} 45$. Elle se trouvait d'un côté ou de l'autre de ce dernier.

\subsubsection{3 $\operatorname{Tn}^{\mathrm{c} m}$}

Courtine (shft) mentionnée dans l'inscription de fondation B-M $257=R E ́ S$ 3022, qu'elle localise entre les bastions $Z r b n$ et $L b^{\prime} n$. Sa construction est commanditée par des membres du sous-clan $Y f^{c} n$, fraction du clan $G b^{\prime} n$ (d'après $M a^{c} \bar{i} n 93 \mathrm{C} / 52$ ), et du sous-clan $D f g n$, fraction d'un clan non identifié. Ces travaux interviennent sous le règne de 'byd' $Y \underline{t}^{\mathrm{c}}$, vers le $5^{\mathrm{e}}$ siècle av. J.-C.

L'inscription de fondation est conservée dans son emplacement d'origine, dans la courtine entre les bastions 54 et 55 . Cette localisation avait déjà été reconnue par Ch. Robin (1979a : fig. 2) et A. Agostini (2011 : fig. 5).

Cette courtine semble également mentionnée dans l'inscription B-M $248=R E ́ S 3010$, remployée dans le bastion 50, qui évoque la courtine $\operatorname{Tn}^{c}[m]$ (shftn $\operatorname{Tn}^{\mathrm{c}}[$.$] ).$

\subsubsection{4 $\mathrm{Ts}^{2} b m(I)$}

Courtine (ṣhft) mentionnée dans l'inscription de fondation du bastion $\underline{d}-M l h$ (bastion 2), B-M $10=R E S S 3012$, datée du règne de 'byd $d^{c} \underline{t}^{c}$ et son fils $W q h^{\prime} l$ Rym, vers les $5^{\mathrm{e}}-4^{\mathrm{e}}$ siècles av. J.C. Cette inscription comporte des lacunes qui ne permettent pas de déterminer la nature de $T s^{2} b m$. Toutefois, deux inscriptions trouvées à proximité, RÉS 3015 (bastion 56, Nmrn) et RÉS 3024 (tour 1, Rbqn), mentionnent l'une $s h f t-s^{1} T\left(s^{2}\right)[\ldots]$ (sa courtine $T s^{2} \ldots$ ) et l'autre « [...] $t^{n}$ $T s^{2} b m »$, vraisemblablement «[s $\left.h f\right] t^{n} T s^{2} b m »\left(\right.$ la [courti]ne $\left.T s^{2} b m\right)$. L'ouvrage $T s^{2} b m$ est donc certainement une courtine (shft).

A cela s'ajoute B-M $267=R E ́ S 3017$ bis qui mentionne une structure attenante à la porte sudouest de la ville sous le nom de $[\ldots] \mathrm{s}^{2} \mathrm{bm}$ que l'on peut restituer en $[T] \mathrm{s}^{2} \mathrm{bm}$.

$R E ́ S 3012,3015,3017$ bis et 3024 ont été trouvées en place ou remployées dans les bastions 1 , 2 et 56. La courtine est censée être proche de la porte sud-ouest, qui est localisée entre les bastions $56(\mathrm{Nmrn})$ et $57\left(\mathrm{Yt}^{\mathrm{c}} \mathrm{n}\right)$. On peut la restituer à cet emplacement, sans toutefois exclure également une localisation entre les bastions 57 et 2 telle qu'envisagée par J.-F. Breton (1994 : fig. 26).

Malgré leurs lacunes, RÉS 3012 et RÉS 3017 bis apparaissent comme les inscriptions de fondation de cette courtine. Sa construction serait donc commanditée par le sous-clan $Y f^{\prime} n$, fraction du clan $G b^{\prime} n$ (d'après Ma'īn $93 \mathrm{C} / 52$ ). 


\subsubsection{5 $\mathrm{Ts}^{2} \mathrm{bm}(\mathrm{II})$}

Courtine (ṣft) mentionnée dans deux inscriptions, B-M $41=R E ́ S 3038$ et B-M 43, respectivement remployées dans le bastion 15 et la courtine 15-16. La date précise des deux inscriptions n'est pas connue (v. $5^{\mathrm{e}}-\mathrm{1}^{\mathrm{er}}$ siècles av. J.-C.).

Compte tenu de la distance qui les sépare, nous faisons l'hypothèse d'une courtine homonyme mais distincte de $T s^{2} b m$ (I) mentionnée à l'autre extrémité du rempart (voir $T s^{2} b m$ (I)). Elle serait à localiser sur le côté nord du rempart, aux environs des bastions 15 et 16 .

\subsubsection{T'rm}

Courtine (ṣhft) mentionnée dans l'inscription de fondation B-M $147=R E ́ S$ 2999. Sa (re)construction est commanditée par des membres du sous-clan Dmrn, fraction d'un clan non identifié. Ces travaux interviennent sous le règne de $W q h^{\prime} l Y \underline{t}^{c}$ et de son fils ' $l y f^{\circ} Y s^{2} r$ (II), dans le dernier quart du $1^{\text {er }}$ siècle av. J.-C. Il s'agirait donc d'une courtine reconstruite à une date tardive de l'occupation du site. L'inscription de fondation est conservée à son emplacement d'origine et permet de localiser la courtine entre les bastions 40-41. Cette localisation a déjà été reconnue par Ch. Robin (1979a : fig. 4).

\subsubsection{Y $\dot{g} l$}

Bastion $(m h f d)$ mentionné dans l'inscription B-M $10=R E ́ S$ 3012, sous le règne de 'byd' $Y \underline{t}^{c}$ et son fils $W q h^{\prime} l$ Rym, vers les $5^{\mathrm{e}}-4^{\mathrm{e}}$ siècles av. J.-C. Les lacunes de l'inscription ne permettent pas de savoir si ce bastion a été bâti à Yathill. Il pourrait s'agir du bastion homonyme du rempart de Qarnā mentionné dans B-M 231 (fragment de Bauer 5).

\subsubsection{Yhr}

Bastion ( $m h f d$ ) mentionné dans l'inscription de fondation B-M 242. L'inscription fragmentaire ne peut pas être précisément datée. Elle est remployée dans le bastion 48 ( $d-B q r n)$. Yhr pourrait être un bastion voisin (n49 ?), ou un nom alternatif, ultérieur, pour le bastion 48.

\subsubsection{Ys $\mathrm{s}^{2} \mathrm{bm}$}

Bastion $(m h f d)$ mentionné dans l'inscription de fondation B-M $280=R E ́ S$ 2976. Sa (re)construction est commanditée par des membres du sous-clan $\dot{G} z r-S^{l} h f n$, fraction d'un clan non identifié. Ces travaux interviennent vers les $5^{\mathrm{e}}-4^{\mathrm{e}}$ siècles av. J.-C. d'après la graphie du texte (Pal.Pi. E2). L'inscription B-M $259=R E S S 2973$ le mentionne probablement lors d'une phase de restauration, sous le règne de $Y \underline{t}^{(')} l S d q$, au début du $1^{\text {er }}$ siècle av. J.-C.

Ces deux mentions apparaissent dans deux inscriptions remployées dans le secteur sud-ouest du rempart (courtine 1-57 et bastion 56). D'après la localisation des remplois, J.-F. Breton (1994: 111) fait l'hypothèse que $Y s^{2} b m$ correspond au bastion $57\left(\mathrm{n}^{\circ} 1\right.$ dans sa propre numérotation). Pour que cette hypothèse soit recevable, il faudrait exclure l'identification du bastion 57 avec $Y \underline{t} n$ (voir l'entrée correspondante). Il s'agit plus vraisemblablement de l'un des bastions non identifiés du secteur sud-ouest de Barāqish.

\subsubsection{Y $\underline{t}^{c} n$}

Bastion $(m h f d)$ mentionné dans l'inscription de fondation B-M $273=R E ́ S$ 2978. Sa (re)construction est commanditée par des membres du sous-clan $S^{2}{ }^{\mathrm{c}} \underline{t}$, fraction du clan $G b^{\prime} n$ (Ma '̄in 95/10-11). L'inscription est conservée dans son emplacement d'origine, dans le bastion 57 et permet d'identifier celui-ci à $Y \underline{t^{c}} n$. Cette localisation a déjà été reconnue par Ch. Robin (1979a : fig. 2). 
D'après sa position dans la maçonnerie, l'inscription RÉS 2978 serait pour J.-F. Breton (1994 : 110) postérieure à $R E S S 3021$, et donc postérieure au règne de ' $l y f^{c} Y s^{2} r$ et de son fils $H f n^{m} R y m$, que $1^{\prime}$ 'on peut dater entre le $3^{\mathrm{e}}$ et le $2^{\mathrm{e}}$ siècle av. J.-C. (Annexe 4).

5.1.1.31 Zbyn

La description de Rbqn (II) s'applique en tout point à Z Zbyn. Il s'agit de l'un des bastions non localisés que liste RÉS 3021 dans le secteur sud-ouest de la ville, peut-être l'un de ceux que nous ne sommes pas parvenus à identifier (bastions $n^{\circ} 50$ à 53). J.-F. Breton l'identifie au bastion $n^{\circ} 56$ sans autre fondement que son apparition dans l'énumération des bastions et courtines de RÉS 3021. Le bastion n56 semblait plus vraisemblablement nommé Nmrn (voir l'entrée correspondante).

\subsubsection{Zrbn}

Bastion $(m h f d)$ mentionné dans l'inscription B-M $257=$ RÉS 3022 comme l'une des extrémités de la courtine $T n^{\prime} m$ (courtine 54-55). Il s'agit donc du bastion 54 ou 55. Nous avons fait l'hypothèse que le bastion 54 était vraisemblablement nommé $L b^{\prime} n$; il remploie dans sa maçonnerie l'inscription B-M 256 qui mentionne la construction de $L b^{\prime} n$ (voir l'entrée $L b^{\prime} n$ (I)). Zrbn serait donc le bastion 55. Cette localisation a déjà été reconnue par Ch. Robin (1979a : fig. 2). Elle diffère des restitutions de J.-F. Breton (1994 : 110-11) et d'A. Agostini (2011 : fig. 5) qui positionnent $L b^{\prime} n$ au bastion 55 et $Z r b n$ au $n^{\circ} 54$.

Le bastion Zrbn existait déjà sous le règne de 'byd' $Y \underline{t}^{\mathrm{c}}$, vers le $5^{\mathrm{e}}$ siècle av. J.-C. (RÉS 3022). Il semble faire l'objet d'une restauration commanditée par des membres du sous-clan $S^{2 c} \underline{t}$, fraction du clan $G b^{\prime} n$ (d'après Ma Mīn 95/10-11) sous le règne de ' $l y f^{c} Y s^{2} r$ (I) et de son fils $H f n^{m}$ Rym, vers les $3^{\mathrm{e}}-2^{\mathrm{e}}$ siècles av. J.-C.

\subsubsection{Essai de mise en ordre chronologique}

L'inscription de Șirwāḥ-Khawlān RÉS 3946, datée du début du VII siècle av. J.-C., est la plus ancienne mention épigraphique d'une fortification de Yathill (Barāqish). Ce rempart est l'œuvre du mukarrib sabéen Karib'īl Watār fils de Dhamar'al̄i. Le sondage extra muros réalisé en 2005-2006 au pied du bastion 7 par F. Fedele a montré que le rempart actuellement visible date exclusivement de la période minéenne du site. Il ne peut en aucun cas être identifié avec la première fortification commanditée par le souverain sabéen (Fedele 2010). F. Fedele propose d'identifier le rempart sabéen au mur F4 (stratum R) de ce sondage, à savoir un mur dont le tracé déborde celui de l'enceinte minéenne et d'une altitude inférieure. Les vestiges de ce mur en pierre sont haut de $105 \mathrm{~cm}$ et épais de $75 \mathrm{~cm}$. Il était surmonté d'une superstructure en briques crues. Il s'agit d'une enceinte modeste dont le rôle aurait été de marquer la limite du site plus que de le défendre (Fedele 2010 : 116). Cette hypothèse se défend d'autant mieux au regard du nombre élevé de villes et bourgades prétendument fortifiées par Karib’̄il Watār fils de Dhamar'alī dans RÉS 3945 et 3946. Il est peu probable que toutes aient bénéficié de solides défenses dans des délais aussi limités. Il s'agit plutôt de la manifestation d'un pouvoir conquérant dont les réalisations architecturales hautement symboliques cherchent à marquer la présence dans le paysage.

Les inscriptions de Barāqish nous renseignent exclusivement sur le rempart minéen, encore visible aujourd'hui. Celui-ci est bâti sur les niveaux de l'occupation d'époque sabéenne, à une 
date qui est postérieure au début du $7^{\mathrm{e}}$ siècle av. J.-C. Se pose la question du début et du rythme de sa construction. On ne peut y répondre sans se heurter à quelques difficultés.

Premièrement, les constructions connues par les textes ne sont pas toujours aisément localisables.

Deuxièmement, les inscriptions ne mettent en lumière que des instantanés de la construction du rempart, mettant l'accent sur les aménagements à proximité de la porte principale (bastions 12 et 54-58), de la poterne et des temples (bastions 43-49). Ce sont les zones de circulation privilégiées, celles qui bénéficient de la plus forte visibilité. Les parties ouest, nord et est demeurent peu documentées.

Troisièmement, les textes ne font pas toujours la différence entre une construction et une restauration de sorte que nous ne pouvons bien souvent les considérer que comme terminus ante quem.

Quatrièmement, la chronologie des inscriptions se fonde quasi exclusivement sur celle des rois de Ma'īn or beaucoup d'incertitudes demeurent. Pour les besoins de cette mise en ordre, on s'appuiera sur une révision de la chronologie royale (Annexe 4).

En dépit de ces contraintes, plusieurs périodes d'interventions sont identifiables et peuvent être mises en perspective (voir la figure 1 qui fait figurer les différentes phases de construction). Si une ébauche en avait été esquissée par J.-F. Breton (1994 : 109-12), il semble utile d'en reprendre les éléments. Nous nous éloignons en effet de sa reconstruction pour deux raisons principales : d'une part, il supposait l'antériorité du règne de ' $l y f^{\complement} Y s^{2} r$ sur ceux de 'byd' $Y \underline{t}^{c}$ et $W q h^{\prime} l$ Rym là où il y aurait postériorité ${ }^{20}$; d'autre part, l'identification de plusieurs bastions et courtines diffèrent (voir 5.1.1 "Les éléments du rempart").

\section{Insérer la figure $1 \mathrm{ici}$.}

$\mathrm{Au}$ total, 37 inscriptions se rapportant à l'édification et à la restauration du rempart ont été identifiées (Annexe 2). Parmi elles, 24 peuvent être associées à un ou plusieurs rois de Ma īn, soit explicitement, soit par recoupement ${ }^{21}$. La succession des règnes permet la reconstruction suivante :

Étape 1 - Une inscription de $\mathrm{Ma}^{\mathrm{c}} \mathrm{in}$ ( $\mathrm{Ma}^{\mathrm{c}} \mathrm{i} \mathrm{n}$ 6), de style paléographique C3 (Pal.Pi.), mentionne la réalisation de trente coudées de maçonnerie dans la muraille de Yathill ( $s^{2} l \underline{t y}$ ' $m h m b$ - $\left.g n^{\prime} Y \underline{t} l\right)$. Aucun souverain n'est mentionné. Seules les inscriptions du règne de Hlkrb $S d q$ bn 'byd' présentent ce style $\mathrm{C} 3$ ce qui tendrait à indiquer une date vers le $6^{\mathrm{e}}$ siècle av. J.-C. Une

\footnotetext{
${ }^{20} \mathrm{La}$ succession de ces règnes se fonde sur la paléographie des inscriptions. Celles du règne de ' $l y f^{\prime} Y s^{2} r$ sont du type D2-D3 défini par J. Pirenne, celles de 'byd ' $Y \underline{t}{ }^{\prime}$ et $W q h^{\prime} l$ Rym du style E2 et E3 (plus rarement E1). Toutefois J. Pirenne a elle-même envisagé de décaler son style paléographique D après le $E$ (Von Wissmann $1976:$ n. 127 p. 373), von Wissmann à sa suite et A. Avanzini (A. Avanzini 1995: 49) s'accordent à considérer le type paléographique $\mathrm{D}$ comme postérieur aux types $\mathrm{E} 2$ et $\mathrm{E} 3$, opinion à laquelle nous nous rangeons.

${ }^{21}$ Les recoupements concernent Y.92.B.A 37 A-B et RÉS 2976 :

Y.92.B.A $37 \mathrm{~A}-\mathrm{B}$ daterait du règne de 'byd ${ }^{\prime} Y \underline{t}^{\prime}$ et de son fils $H y w^{m}$ par association à Y.03.B.R44-45.2bis +2 ter + 2. Toutes deux se rapportent à l'édification du bastion $\underline{d}-N d b n$ et de la courtine $T f s^{2}$.

RÉS 2976 daterait du règne de Wqh'l Rym par association à Y.05.B.B 13. Toutes deux sont du style paléographique E2, se rapportent à des constructions prises en charge par la fraction de clan des $\dot{G} z r-S^{\prime} h f h$ et évoquent un certain Rtd l'l (bn Wdd'l) $\underline{d}-\dot{G} z r S^{\prime} h f n$.
} 
inscription antérieure, RÉS 2947, datée du $6^{\mathrm{e}}$ siècle par la graphie (Pal.Pi. C1), est remployée dans le rempart et mentionne le roi de Ma'īn 'lyf $R y m$ (II). Si le remploi et le contenu fragmentaire du texte ne permettent pas de l'associer à une étape de construction du rempart, on ne peut toutefois pas exclure une mise en chantier de la fortification sous ce règne.

Étape 2 - Règne de ' $l y f^{c} W q h$ en corégence avec son fils $W q h^{\prime} l S d q$ (v. 5 siècle av. J.-C.) : première intervention connue dans les inscriptions de Barāqish (B-M $247+255$, graphie Pal.Pi. E1). Une courtine est aménagée, probablement dans le secteur des bastions 50-52 (fig. 1, en rose).

Étape 3 - Règnes successifs de 'byd $Y \underline{t}^{c}$ seul, en corégence avec son fils $H y w^{m}$, en corégence avec son fils $W q h^{\prime} l$ Rym et $W q h^{\prime} l$ Rym seul (v. $5^{\mathrm{e}}$-début $4^{\mathrm{e}}$ siècles av. J.-C.) : la majorité des interventions datent de cette période, avec 13 inscriptions de styles paléographiques E1, E2 et E3 (Pal.Pi.) ${ }^{22}$. Sont mentionnées les constructions des bastions $\underline{d}-H f n, \underline{d}-M l h, \underline{d}-N d b n, L b^{2} n$ (I) et (II), $S d q n, Y \dot{g} l, Y s^{2} b m$ et $Z r b n$ et des courtines $M d \underline{d} b, S^{2} b m t, T f s^{2}, T n^{c} m$ et $T s^{2} b m$ (I). Ils se répartissent dans le secteur de la porte sud-ouest, entre les bastions 54 et 2, et plus au sud, entre les bastions 41 et 45 , le long du temple de Nakrah (fig. 1, en rouge). Cet ensemble donne le sentiment d'un aménagement d'une large partie méridionale du rempart.

Étape 4 - Règnes de 'bkrb $S d q$ et de 'byd' Rym fils de $H y w S d q$ (v. $4^{\text {e }}$ siècle av. J.-C.) : aucun argument décisif ne permet de savoir lequel précède l'autre. Ils semblent régner peu après deux successeurs de $W q h^{\prime} l$ Rym qui sont $H f n S d q$ et ' $l y f^{\circ} Y f S^{2}$ (Annexe 4). Quatre inscriptions de style paléographique E3 (Pal.Pi.) commémorent la construction d'au moins trois bastions ( $\underline{d}$-Bqrn, $\left.\underline{d}-N d b n, \underline{d}-S^{2} f t n\right)$ et deux courtines $\left(S^{1} l f, D d n\right)^{23}$. Ces constructions semblent s'inscrire dans la continuité de celles entreprises à l'étape 3 en poursuivant l'édification du rempart entre les bastions 45 et 51, faisant de la sorte la jonction avec le secteur de la porte sud-ouest (fig. 1, en bordeaux).

Des travaux sont également entrepris sur le côté nord du rempart (courtine $D d n$ ) et probablement à l'est (courtine 31-32). Il est probable que l'ensemble du site ait été alors progressivement ceint.

Étape 5 - Règnes de ' $l y f^{\complement} Y s^{2} r$ (I) en corégence avec son fils $H f n^{m} R y m$ puis avec son fils $W q h^{\prime} l$ $N b t$ et règne de $W q h^{\prime} l N b t$ seul (v. $3^{\mathrm{e}}-2^{\mathrm{e}}$ siècles av. J.-C.) : trois inscriptions relatent des réaménagements substantiels de l'ensemble du secteur de la porte sud-ouest ${ }^{24}$, depuis le bastion 2 à l'ouest jusqu'au 54 à l'ouest et probablement au-delà, jusqu'au bastion 50 (fig. 1, en vert). Outre la restauration d'au moins huit bastions et deux courtines (bastions [.]r'n; $d$-Mlh; Lb'n (I) ; Rbqn (II) ; Rdwwn ; Y $\underline{t}^{c} n$; Zbyn ; Zrbn et courtines $\underline{d}$-Hṣbr et $H r s^{2}$ ), la tour Rbqn (I) est bâtie en avant du bastion 2 et reliée à ce dernier par un passage maçonné.

\footnotetext{
${ }^{22} \mathrm{~B}-\mathrm{M} 10=R E ́ S 3012 ; \mathrm{B}-\mathrm{M} 180=R E ́ S 2942 ; \mathrm{B}-\mathrm{M} 182=R E ́ S 2929+2941+2945+2946$; B-M $211+\mathrm{B}-\mathrm{M}$ $204=R E ́ S 2952+2949 ; \mathrm{B}-\mathrm{M} 222=R E ́ S 3005 ; \mathrm{B}-\mathrm{M} 238=R E ́ S 3535 ; \mathrm{B}-\mathrm{M} 256=R E ́ S 2972+2970+2971+$ 2971 bis $\mathrm{B}+2971$ bis A ; B-M $257=R E ́ S 3022$; B-M $280=R E ́ S$ 2976; Y.03.B.R44-45.2bis +2 ter +2 ; Y.05.B.B.13; Y.92.B.A $21+30$; Y.92.B.A 37 A-B.

${ }^{23} \mathrm{~B}-\mathrm{M} 56=R E S S 3060 ; \mathrm{B}-\mathrm{M} 103=$ Robin-Barāqish $80 ; \mathrm{B}-\mathrm{M} 241=R E ́ S 2965 ; \mathrm{B}-\mathrm{M} 249$.

${ }^{24} \mathrm{~B}-\mathrm{M} 272=R E ́ S 3021 ; \mathrm{B}-\mathrm{M} 1=R E ́ S 2975 ; \mathrm{B}-\mathrm{M} 1=R E ́ S 2975$.
} 
Une inscription supplémentaire, B-M 108, évoque la (re)construction de la courtine 'brm sous le règne de $W q h^{\prime} l$. Au moins cinq rois de $\mathrm{Ma}^{\text {cinn }}$ portent ce nom ${ }^{25}$. Toutefois, le style paléographique se rapproche le plus de celui des inscriptions du règne de $\mathrm{Wqh} l \mathrm{Nbt}$.

Étape 6 - Règnes de $Y \underline{t}^{c \prime} l S d q$ puis de son fils $W q h^{\prime} l Y \underline{t}^{c}$ en corégence avec son fils ' $l y f^{\circ} Y S^{2} r$ (II) ( $1^{\text {er }}$ siècle av. J.-C.) : de cette époque datent les deux dernières interventions connues par les inscriptions ma īniques (respectivement B-M $259=R E ́ S 2973$ et B-M $147=R E ́ S$ 2999). Il s'agit là d'opérations limitées. L'une d'elle (B-M $147=R E ́ S$ 2999) intervient après le passage de l'expédition romaine menée par Ælius Gallus et s'explique peut-être par des destructions occasionnées par l'armée romaine.

En résumé, nous aurions une fortification dont le chantier aurait débuté au cours du $6^{\mathrm{e}}$ siècle, sous l'impulsion de Minéens établis à Qarnā (actuelle Ma ${ }^{\top} \bar{i} n-M^{c} \bar{i} n$ 6). Le chantier prend progressivement de l'ampleur tout au long du $5^{\mathrm{e}}$ siècle pour s'achever, semble-t-il vers la fin $\mathrm{du} 5^{\mathrm{e}}$ - début du $4^{\mathrm{e}}$ siècle. Si le pic d'activité correspond aux règnes de 'byd ${ }^{\mathrm{c}} \underline{t}^{\mathrm{c}}$ et de son fils $W q h^{\prime} l S d q$, presque tous les souverains connus entre le règne de 'lyf $W q h$ et celui de 'byd' $R y m$ sont concernés (déb. $5^{\mathrm{e}}$-fin $4^{\mathrm{e}}$ siècle av. J.-C.).

La restitution en plan (fig. 1) semble indiquer une priorité donnée à l'aménagement des secteurs de la porte et de la poterne, reliés entre eux dans un second temps, avant que ne soit mis en œuvre le reste du chantier, vers le nord. L'élaboration de ces grands traits demande désormais à être confrontée à une étude minutieuse de l'appareil du rempart pour en valider la séquence.

\subsubsection{Pour une datation absolue de la mise en chantier}

Une difficulté réside dans le fait que les premières inscriptions connues évoquant le rempart pourraient être postérieures au début de la mise en chantier du rempart. Barāqish passe sous le contrôle des rois de $\mathrm{Ma}^{\mathrm{c}} \overline{\mathrm{in}}$ dès le $7^{\mathrm{e}}$ siècle av. J.-C. or les premiers textes ne semblent dater que du $6^{\mathrm{e}}$ siècle av. J.-C.

Les datations ${ }^{14} \mathrm{C}$ obtenues dans le sondage extra muros du secteur $\mathrm{C}$ apportent sur ce point quelques éclairages (Fedele 2010 : fig. 136 et table 3).

Un premier échantillon (sample 200) provient d'un niveau qui précède la tranchée de fondation du bastion 7 .

Un second échantillon (sample 191) provient d'un niveau qui s'appuie contre la neuvième assise du bastion, à l'interface entre la fondation du rempart et son élévation.

Les dates radiocarbones obtenues sont :

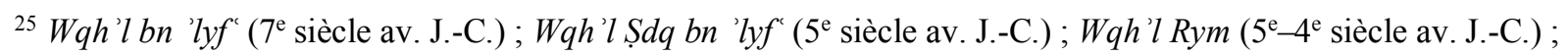
$W q h^{\prime} l N b t\left(3^{\mathrm{e}}-2^{\mathrm{e}}\right.$ siècle av. J.-C. $) ; W q h^{\prime} l \underline{t}^{\prime}\left(1^{\mathrm{er}}\right.$ siècle av. J.-C.). 


\begin{tabular}{|c|c|c|c|c|c|c|}
\hline Lab \# & $\begin{array}{l}\text { Échantillo } \\
\text { n }\end{array}$ & $\begin{array}{l}\text { Localisatio } \\
\text { n }\end{array}$ & Nature & $\begin{array}{l}\text { Age } \\
\text { radiocarbo } \\
\text { ne (yrs BP) }\end{array}$ & $\begin{array}{l}\text { Date } \\
\text { calibrée (2 } \\
\text { sigma) } \\
\text { Début-fin } \\
\text { (probabilit } \\
\text { é) }\end{array}$ & $\begin{array}{l}\text { Median } \\
\text { Probabili } \\
\text { ty }\end{array}$ \\
\hline $\begin{array}{l}\text { DSH29 } \\
8\end{array}$ & 200 & $\begin{array}{l}\text { Base de la } \\
\text { tranchée de } \\
\text { fondation }\end{array}$ & $\begin{array}{l}\text { Charbon de } \\
\text { bois } \\
\text { (indétermin } \\
\text { é) }\end{array}$ & $2486 \pm 34$ & $\begin{array}{l}\text { cal BC 782- } \\
482(0.995) \\
\text { cal BC 441- } \\
434(0.005)\end{array}$ & -634 \\
\hline $\begin{array}{l}\text { DSH86 } \\
2\end{array}$ & 191 & $\begin{array}{l}\text { Interface } \\
\text { fondation / } \\
\text { élévation }\end{array}$ & $\begin{array}{l}\text { Charbon de } \\
\text { bois } \\
\text { (indétermin } \\
\text { é) }\end{array}$ & $2439 \pm 25$ & $\begin{array}{l}\text { cal BC 749- } \\
684(0.25) \\
\text { cal BC 667- } \\
639(0.08) \\
\text { cal BC 590- } \\
576(0.02) \\
\text { cal BC 571- } \\
408(0.65)\end{array}$ & -528 \\
\hline
\end{tabular}

En dépit des limites inhérentes à ces résultats ${ }^{27}$, ces deux dates combinées aux autres datations du sondage faisaient dire à F. Fedele :

"This supports a completion of the Minaean wall and its accessories during the 6th or 5th century BC" (Fedele 2010 : 134).

Nous en tirons plusieurs enseignements :

1/ Ces dates sont cohérentes avec la reconstruction historique proposée : un chantier qui s'étale principalement aux $6^{\mathrm{e}}-5^{\mathrm{e}}$ siècles av. J.-C.

2/ Elles vont dans le sens d'une datation du règne de 'byd $Y \underline{t}^{c}$, durant lequel une majeure partie du rempart fut bâtie, au $5^{\mathrm{e}}$ siècle av. J.-C. plutôt qu'au $4^{\mathrm{e}}$ siècle av. J.-C. (voir Annexe 4 « 'byd' $Y \underline{t}^{c}$, roi de $\mathrm{Ma}{ }^{\mathrm{i}} \mathrm{i} n \mathrm{du} 5^{\mathrm{e}}$ siècle av. J.-C. »).

3/ Les fourchettes des datations ne sont certes pas assez précises pour déterminer si le chantier du rempart minéen a pu débuter dès le passage de la ville sous l'autorité des rois de Ma în, dans le courant du $7^{\mathrm{e}}$ siècle av. J.-C. Cette hypothèse semble toutefois peu probable car le sondage $\mathrm{du}$ secteur $\mathrm{C}$ montre qu'une phase d'occupation durable sépare l'abandon de la "Earlier Sabaean Occupation (c. 820-700 BC) » associée au mur F4, possiblement le rempart sabéen, de la construction du rempart minéen. Cette phase intermédiaire est datée par F. Fedele (2010) vers 650-600/550 av. J.-C. Bien que nommée «Later Sabaean Occupation » par la MAIRY, nous sommes déjà à un moment où les rois de Ma'īn avaient autorité sur Yathill (voir « 4.2 L'alliance des tribus de $\mathrm{Ma}^{\text {c}} \bar{n}$ et Yathill... »).

\footnotetext{
${ }^{26}$ Les dates sont calibrées avec le logiciel de calibration radiocarbone Calib Rev 7.10, référence de calibration (Reimer et al. 2013).

${ }^{27}$ Faute d'identification de la nature du bois analysé, on ne peut exclure un effet « vieux bois », qui vieillirait artificiellement la date. L'autre limite est l'effet de plateau de la courbe de calibration qui ne permet pas d'obtenir de résolution chronologique fine. Voir sur ce point Fedele 2010 : 106.
} 
Les trois arguments dont on dispose pour dater le début du chantier dans le courant du $6^{\mathrm{e}}$ siècle sont :

- Les datations ${ }^{14} \mathrm{C}$;

- L'inscription Ma īn 6 (style Pal.Pi. C3, v. $6^{\mathrm{e}}$ siècle av. J.-C.) qui évoque la construction de 30 coudées du rempart de Yathill;

- La fouille du temple de Nakrah qui a montré que le parement externe de la courtine 4445 existait déjà durant la phase la plus ancienne du temple (Minéen C) (De Maigret \& Robin 1993: 453), dont l'occupation est associée à une inscription de style paléographique C2 (v. $6^{\mathrm{e}}$ siècle av. J.-C.) (voir « 4.2.1 - Brn, temple de Nakrah à Barāqish »).

\subsection{Les temples}

Les sources épigraphiques nous renseignent sur la présence de cinq temples; quatre sont identifiés et deux ont été fouillés.

\subsubsection{Brn, temple de Nakrah à Barāqish}

Le temple de Nakrah est localisé à proximité du rempart. La MAIRY l'a intégralement fouillé et restauré, avant qu'il ne fût détruit par des bombardements de l'aviation saoudienne en 2015 (De Maigret 1991a ; 1991b ; 1993 ; 2004 ; 2010 ; De Maigret \& Robin 1993 ; Robin \& De Maigret 2009). Ce temple apparaît parfois sous le nom de temple A. Sa chronologie, son identification et ses principaux textes ont été l'objet d'une étude détaillée (De Maigret \& Robin 1993). L’essentiel est ici synthétisé et quelques éléments sont actualisés.

L'inscription Y.92.B.A $21+30$ provenant du temple de Nakrah rapporte la construction d'un chancel $\left(s^{3} n f n\right)$ dans le temple Brn par des membres de la fraction $B l h$ du clan $G b^{\prime} n$, sous le règne de 'byd ${ }^{c} \underline{t}^{c}$ (v. $5^{\mathrm{e}}$ siècle av. J.-C.). Ce texte nous donne le nom antique du temple, que Ch. Robin vocalise Barrān. On le retrouve mentionné dans d'autres inscriptions provenant du temple : Y.92.B.A 14 et 15, ainsi probablement que RÉS 2959.

La divinité vénérée était Nakrah ou Nakraḥ le Patron (Nkrh $\left.S^{2} y m n\right)$ toutefois, d'autres divinités y font l'objet de dédicace : 'Athtar dhū-Qabḍ, Wadd et Nakrah (Y.92.B.A 37 A-B), Shahr et Ma'ān ( $S^{2} h r$ et $M^{c} n$ - Y.03.B.A.1), 'Athtar dhū-Yahriq (Y.92.B.A 10 et 17), 'Athtar Shariqān (Y.92.B.A 46), Kahlān Nabaṭ'athtar (Khln Nbțt ț - Y.92.B.A 28). Il se peut que certains documents ne soient pas en place et proviennent de temples de voisins. Néanmoins cela reflète un lieu de culte non exclusif.

La fouille a montré trois phases successives de construction (De Maigret \& Robin 1993 : 44858).

Le niveau Minéen C (v. $6^{\mathrm{e}}$ siècle av. J.-C.), le plus ancien, correspond à la construction du temple. On peut faire l'hypothèse que l'inscription Y.92.B.A 15 date de cette période. Remployée dans la terrasse sud du niveau tardif Minéen A, c'est le plus ancien texte connu dans le temple d'après la paléographie (voir notamment De Maigret \& Robin 1993 : 474). Il est 
de style C2 (Pal.Pi.), datable vers le $6^{\text {e }}$ siècle av. J.-C. ${ }^{28}$. L'inscription évoque une taxe sur les animaux introduits dans l'avant-cour ( $s r h t$ t) et dans l'édifice ( $h t b)$ Barrān ${ }^{29}$. Sur la base de parallèles architecturaux, A. de Maigret proposait également de dater cette phase des $7^{\mathrm{e}}-6^{\mathrm{e}}$ siècles av. J.-C. (De Maigret \& Robin 1993 : 455).

Une datation radiocarbone confirme cette fourchette chronologique (Robin \& De Maigret 2009 : 67 ; voir chap. 16, table A : sample Y.92.B/135).

Le niveau Minéen B (v. $5^{\mathrm{e}}$ siècle av. J.-C.) se caractérise par l'aménagement d'un avant corps et une réorganisation interne. Des aménagements secondaires y sont réalisés pendant le règne de 'byd' $\underline{t}^{c}$ (Y.92.B.A $21+30$ ). Outre Y.92.B.A $21+30$ déjà évoquée, quatre inscriptions rédigées par $B s^{l} l$ fils de $M^{c} s^{l}$ de la fraction $S^{2} \underline{t} m$ du clan $G b^{3} n$ se rapportent à des aménagements qui sont, d'après la fouille, contemporains de celui du chancel et donc du règne de 'byd $Y \underline{t}^{c}$ (v. $5^{\mathrm{e}}$ siècle av. J.-C.) : aménagement de banquettes ( $t w \underline{t} b$, Y.90.B.A.7), offrande d'un brûle-encens (Y.92.B.A 46), d'une stèle (Y.90.B.A 18), d'un élément architectural ( $m w s^{3} l t$, Y.92.B.A $32+$ $5)^{30}$.

Le niveau Minéen A se caractérise par le réaménagement du parvis et la construction de l'annexe $\mathrm{C}$ au sud du temple. Les datations radiocarbones montrent que ce niveau caractérise l'occupation du temple durant toute la seconde moitié du $1^{\mathrm{er}}$ millénaire av. J.-C. (voir chap. 16, table A dans cet ouvrage). Ces réaménagements pourraient correspondre aux importants travaux évoqués dans l'inscription $R E ́ S 2980$ bis, sous la corégence de $Y \underline{t}^{(')} l R y m$ et de son fils $T b^{c} k r b$, que nous situons cinq générations après 'byd $Y \underline{t}^{c}$, vers le $4^{\mathrm{e}}$ siècle av. J.-C.

L'occupation se poursuit jusqu'aux $1^{\text {er }}$ siècles av./ap. J.-C. d'après le mobilier exhumé et les datations radiocarbones (Antonini 1999 : 67).

De manière générale, cette chronologie ne s'éloigne guère de celle proposée par Ch. Robin en 1993 (De Maigret \& Robin 1993 : 471-75), excepté dans les datations absolues que permettent les analyses ${ }^{14} \mathrm{C}$.

\subsubsection{Byn, temple de dhū-Samāwī à Barāqish (?)}

Sur le site voisin de Haram, l'inscription Haram 10, datée du tournant de l'ère chrétienne, est une confession des membres des tribus arabes de Amīr et 'Athtar alors qu'elles trouvèrent refuge à Yathill pendant une guerre avec le Haḍramawt et y effectuèrent un pèlerinage à dhūSamāwī.

Parallèlement, l'inscription Y.92.B.A 20 découverte dans le temple Brn de Nakrah, et également datée du tournant de l'ère chrétienne, commémore l'offrande de deux statues à dhuSamāwī dans le temple Byn. Ch. Robin propose deux interprétations possibles (De Maigret \& Robin 1993 : 475): Soit le temple Brn de Yathill a été renommé Byn dans les dernières décennies de son occupation et consacré au culte de dhu-Samāwī, dieu d'Amīr. Cette hypothèse implique l'installation pérenne de tribus arabes à Barāqish après l'effondrement du royaume de Ma īin, à l'instar de ce qui s'observe à Haram (Robin 1991). Soit l'inscription Y.92.B.A 20 provient de la ville de Haram où la présence d'un temple Byn consacré à dhu-Samāwī est par

\footnotetext{
${ }^{28}$ Ce style paléographique caractérise plusieurs inscriptions du mukarrib de Qatabān $H w f^{\prime} m$ Yhn ' $m$ fils de $S^{1} m h w t r$ dont le père serait mentionné dans l'une des dernières inscriptions des mukarribs de Saba', RÉS 3943, vers la fin $7^{\mathrm{e}}-6^{\mathrm{e}}$ siècle av. J.-C. (Robin $2016 \mathrm{~b}: 84-85$ ).

${ }^{29}$ Concernant les termes désignant les différentes parties du temple, on se reportera à Robin 2016b : 461-69.

${ }^{30} \mathrm{Il}$ est d'autant plus probable que $B s^{1} l$ fils de $M{ }^{\prime} S^{1}$ soit le contemporain du roi 'byd ' $Y \underline{t}{ }^{\prime}$ si l'on considère que son fils est un contemporain du roi $W q h^{\prime} l$ Rym, fils de 'byd ' $Y \underline{t}^{\prime}$ (Y.90.B.ext 2).
} 
ailleurs attestée. Nous privilégions la première hypothèse compte tenu de la reconversion qui affecte également le temple voisin de $Q b d^{m}$ au tournant de l'ère chrétienne.

\subsection{3 $Q b d^{m}$, temple de 'Athtar à Barāqish}

Le temple de 'Athtar dhu-Qabḍ ${ }^{\text {um }}$, initialement nommé Temple B par la MAIRY, est bâti immédiatement au nord du temple de Nakraḥ. Il a été intégralement fouillé par la MAIRY (Agostini 2011 ; 2015). Le temple portait le nom de Qabḍ ${ }^{\text {um }}$ ce dont témoigne aussi bien l'épithète de la divinité qui y est vénérée que la mention explicite du «temple Qabḍu ${ }^{\text {um }}$ (byt $\left.Q b d^{m}\right)$ dans l'inscription Y.05.B.B.13/5, découverte in situ.

Y.05.B.B.13 commémore la construction du temple. Les travaux sont coordonnés par un membre de la fraction de clan $\dot{G} z r-S^{\prime} h f n$, chef des maçons et du transport ( $k b r$ grbn $w$-nqln), sous le règne de $W q h^{\prime} l$ Rym, vers la fin du $5^{\mathrm{e}}$-début $4^{\mathrm{e}}$ siècle av. J.-C.

La présence d'une dédicace à Halfān (Y.05.B.B 16) découverte dans ce temple (Agostini 2015) souligne les changements qui s'opèrent sur le site au tournant de l'ère chrétienne. Halfān, tout comme dhu-Samāwī, sont les divinités vénérées par la tribu arabe d'Amīr, originaire de la région de Najrān. La présence de cette tribu est bien attestée à partir du $2^{\mathrm{e}}$ siècle av. J.-C. dans le Jawf, en particulier dans la ville de Haram (Robin 1991). Or au tournant de l'ère chrétienne, des inscriptions sont dédiées à dhu-Samāwī et Halfān dans les deux temples principaux de Yathill. Il est vraisemblable qu'à la suite du passage de l'expédition d' Ælius Gallus, la ville de Yathill ait connu un renouvellement de sa population avec l'introduction d'éléments venus du nord.

\subsubsection{Byt $W d^{m}$, temple de Wadd à Barāqish}

La construction d'un temple consacré à $\operatorname{Wadd}^{u m}\left(\right.$ byt $\left.W d^{m}\right)$ est commémorée par l'inscription B-M $277=R E ́ S 3019$. Le commanditaire des travaux est $B s^{l} l$ fils de $M^{c} s^{1} \underline{d}-S^{2 c} \underline{t} m$ (clan $\left.G b^{\prime} n\right)$, l'un des bienfaiteurs du temple de Nakrah, connu par plusieurs inscriptions et actif sous le règne de 'byd' $Y \underline{t}^{\mathrm{c}}$ au $5^{\mathrm{e}}$ siècle av. J.-C.

A. Agostini (2011 : 53-54) propose d'en localiser les vestiges immédiatement au nord du temple de 'Athtar dhu-Qabḍ ${ }^{u m}$.

\subsubsection{Yhrq, temple de 'Athtar à Shaqab al-Manașșa}

Le sanctuaire antique de Shaqab al-Manaș̣a est implanté à $2,5 \mathrm{~km}$ au sud-sud-ouest de Barāqish, en bordure du périmètre irrigué antique, sur un affleurement gréseux, à proximité d'une zone de redistribution des eaux d'irrigation.

Ce sanctuaire, composé de deux grandes salles hypostyles, était consacré au culte de 'Athtar dhū-Yahriq (Breton et al. 1979 : 425-27 ; Gnoli 1993). L'épithète de la divinité indique le nom du sanctuaire : Yahriq/Yuhariq (Yhrq). Il apparaît explicitement dans la locution "Athtar dieu de Yhrq" (Shaqab 8: 'ttr 'l Yhrq).

La graphie des inscriptions provenant du sanctuaire indique une activité entre les $8^{\mathrm{e}}$ et $2^{\mathrm{e}}$ siècle av. J.-C. Le portique est remis à neuf sous les règnes de 'bkrb $S d q$ fils de $W q h^{\prime} l$ (Shaqab 4) et de son successeur $Y \underline{t}^{(')} l$ Rym (Shaqab 5), vers le $4^{\mathrm{e}}$ siècle av. J.-C.

'Athtar dhū-Yahriq est la divinité tutélaire de la tribu de Yathill. Les dédicaces à cette divinité sont uniquement effectuées à Barāqish, dans le temple de Nakrah (Y.92.B.A 10, Y.92.B.A 16, Y.92.B.A 17), où siège la congrégation (qhlt) de 'Athtar dhū-Yahriq (Y.90.B.A 3), et surtout dans le temple Yahriq de Shaqab al-Manașa (Shaqab 3, 6, 7, 16). La localisation extra muros du sanctuaire de la divinité en facilite l'accès à l'ensemble des membres de la tribu de Yathill. 
Les sacrifices offerts à la divinité lors de son festival (' $h d ̣$ ) sont fréquemment commémorés sur le rempart de la ville (B-M 12, 44, 182, 211, 267).

\subsubsection{F ț $^{\mathrm{c}} n$, sanctuaire de Nakrạ̣ à Darb aṣ-Șabī}

Darb aṣ-Ṣabī se trouve moins de $2 \mathrm{~km}$ à l'ouest de Barāqish. Il s'agit d'une vaste surface de $500 \times 300 \mathrm{~m}$ bornée de neuf stèles (MAFRAY-Darb aṣ-Ṣabī 4A-H, MAFRAY-Darb aṣ-Ṣabī 15) délimitant un espace sacré, un haram consacré à la divinité Nakrah. Sur une quarantaine d'édifices dénombrés, trois ont été étudiés (Robin et al. 1981). Ils comprennent généralement une cour et une ou plusieurs pièces hypostyles. L'une de ces pièces, "Édifice 2", a été reconnue comme temple de Nakrah (Robin et al. 1981: 259). L'ensemble constitue un complexe religieux dédié à Nakrah, comprenant des locaux pour l'hébergement d'un clergé et de fidèles. Nous avons vu précédemment que le nom antique de ce périmètre sacré était vraisemblablement Ft́ ${ }^{c} n$ (MAFRAY-Darb aṣ-Șabī 1 - Robin et al. 1988 : 92).

Le sanctuaire semble avoir connu une longue période de fréquentation, la graphie des inscriptions couvrant la période des $8^{\mathrm{e}}-2^{\mathrm{e}}$ siècles av. J.-C. L'inscription la plus ancienne d'après sa graphie, MAFRAY-Darb aṣ-Șabī 13, est rédigée en saba'ique vers le $8^{\mathrm{e}}$ siècle av. J.-C. Elle mentionne la construction d'un portique. La plupart des inscriptions, de graphie D, datent l'activité principale du sanctuaire de la période minéenne ( $3^{\mathrm{e}}-2^{\mathrm{e}}$ siècles av. J.-C.). Consacré au culte d'une divinité minéenne et ne présentant pas de texte récent, le sanctuaire semble abandonné tandis que disparaît le royaume de Ma ${ }^{c} \bar{i} n$ ( $1^{\text {er }}$ siècle av./ap. J.-C.).

On peut s'interroger sur les raisons de la présence de deux sanctuaires consacrés à Nakrah faisant l'objet, tous deux, de confessions et d'actes de pénitence. A. Agostini (2012:6) l'explique par la fonction : le sanctuaire de Darb aṣ-Șabī est une destination de pèlerinage qui doit permettre d'offrir un refuge, des conditions d'accessibilité et de prophylaxie que n'offre pas le petit temple intra muros de Nakrah. Il évoque également l'hypothèse de temples réservés à des classes sociales distinctes. Cette hypothèse semble moins convaincante si l'on considère que les deux temples comportent des dédicaces de personnes de même extraction sociale. Ainsi, des membres de la fraction $\dot{G} r b t$ du clan $M w q h$ font des offrandes dans les deux temples de Nakrạ̣, à Yathill (Y.92.B.A 37 A-B) et Darb aṣ-Șabī (MAFRAY-Darb aṣ-Ṣabī 3). En revanche, il est possible que le temple de Darb aṣ-Ṣabī ait été ouvert à l'ensemble de la population du royaume de Ma'īn là où l'accès du temple intra muros était avant tout restreint aux habitants de Yathill.

Outre la grande accessibilité du sanctuaire du Darb aṣ-Ṣabī, celui-ci nous semble également se distinguer par deux autres traits : d'une part, son antériorité sur le temple intra muros de Nakrah. Il lui semble antérieur d'au moins un siècle; d'autre part il ne présente aucune inscription royale ni même aucune mention de souverain sabéen ou minéen. En ce sens, le sanctuaire apparaît comme l'un des rares lieu d'asile où le pouvoir royal ne se manifeste - voire ne peut se manifester - en aucune manière.

\subsection{Les structures hydrauliques et agricoles}

Les inscriptions commémorent à de rares occasions l'aménagements d'ouvrages hydrauliques et en réglementent l'usage. Ce sont :

- Des vannes $\left(m^{\prime} h \underline{d} \underline{d}\right)$ et canaux $\left(m t y^{\prime}\right)$ dont l'inscription MAFRAY-Ḥuṣn Āl Șāliḥ 1 réglemente l'usage vers le $8^{\mathrm{e}}$ siècle av. J.-C. 
- Le puits ( $\left.b^{\prime} r\right) T \dot{g} l$ creusé par un membre du clan $G b^{\prime} n$ sous les règnes de 'byd $Y \underline{t}^{c}$ et $W q h^{\prime} l$ Rym (5 ${ }^{\mathrm{e}}$ siècle av. J.-C. - RÉS 2952 + 2949).

- Le réservoir $\left(m^{3} h \underline{d} d n\right) S^{2} b^{\complement} n$ commandité par le souverain minéen $W q h^{\prime} l$ Rym $\left(5^{\mathrm{e}}-4^{\mathrm{e}}\right.$ siècle av. J.-C. - Shaqab 18).

- Les répartiteurs $(m z f)$ de $B h r n$ réparés sous les règnes de ' $l y f^{c} Y s^{2} r$ et de son fils $H w f^{\prime} t t$ (v. $3^{\mathrm{e}}-2^{\mathrm{e}}$ siècles av. J.-C.). Ces travaux sont menés par des membres des sous-clans $\mathrm{Hbrr}$ et $S^{l} y l$, fractions du clan ' $l y ' l$.

\subsection{L'habitat}

Les dernières formes de réalisations architecturales commémorées par des inscriptions sont les constructions de cinq résidences. Quatre sont bâties aux $5^{\mathrm{e}}-4^{\mathrm{e}}$ siècles av. J.-C., sous les règnes successifs de 'byd' $\underline{t}^{c}$ et $W q h^{\prime} l$ Rym :

- $\quad$ Byt ?, pour des membres du sous-clan Blh, fraction du clan Gb'n (Y.03.B.R44-45.3);

- $\quad$ Byt N'mn, pour des membres du clan Gb’n $(R E ́ S 2952+2949)$;

- Byt $S d q n$, pour des membres du sous-clan $S^{2 c} t m$, fraction du clan $G b^{\prime} n$ (YM 26117);

- Byt $Y^{c} d$, pour des membres du sous-clan $S^{2 c} \underline{t}$, fraction du clan $G b^{\prime} n$ (YM 26117);

La cinquième, byt $M h d \underline{d} r$, est bâtie au $1^{\mathrm{er}}$ siècle av. J.-C. sous les règnes de $Y \underline{t}^{\mathrm{\prime}} l S d q$ et son fils $W q h^{\prime} l \underline{t}^{c}(R E ́ S 3016)$.

\subsection{L'activité de construction}

\subsubsection{Quand?}

Au total, 52 inscriptions de construction ont été identifiées au sein du corpus (Annexe 2).

Elles ont principalement trait à la construction du rempart (36), dans une moindre mesure aux temples et à leur aménagement (8), à des résidences (5) et à des aménagements hydrauliques (5).

Elles confortent les observations faites à propos du rempart :

La monumentalisation de Barāqish débute vers le $6^{\text {e }}$ siècle av. J.-C. par la mise en chantier du rempart, sous l'impulsion de Minéens établis à Qarnā (Ma'īn 6), et celle du temple de Nakrah. La monumentalisation du site s'intensifie au cours du $5^{\mathrm{e}}$ siècle av. J.-C., sous les règnes de 'byd' $Y \underline{t}^{c}$ seul, en corégence avec son fils Wqh'l Rym puis sous le règne de ce seul Wqh'l Rym: réaménagement du temple intra muros de Nakrah, construction du temple de 'Athtar dhuQabḍ ${ }^{\text {um }}$, construction du temple de Wadd, construction de la majeure partie du rempart ${ }^{31}$ (fig. 1), construction des résidences $N^{c} m n$, $S d q n$ et $Y^{c} d$, creusement de puits, aménagement de citernes.

Yathill est un chantier permanent qui se poursuit au cours du $4^{\mathrm{e}}$ siècle av. J.-C., sous les règnes de 'bkrb $S d q, Y \underline{t}^{\prime \prime} l$ Rym et 'byd' Rym : nouveaux aménagements dans le temple de Nakrah, achèvement du rempart autour de Yathill (en particuliers les courtines $S^{l} l f$ et $D d n$ et les bastions $\underline{d}-B q r n, \underline{d}-N d b n$ et $\left.\underline{d}-S^{2} f t n\right)$, remise à neuf du portique du temple de 'Athtar Yahriq à Shaqab alManașșa.

Entre le $3^{\mathrm{e}}$ et le $2^{\mathrm{e}}$ siècles av. J.-C., les interventions se limitent à des travaux de fortifications avec un réaménagement en profondeur du secteur sud-ouest du rempart sous les règnes de 'lyf

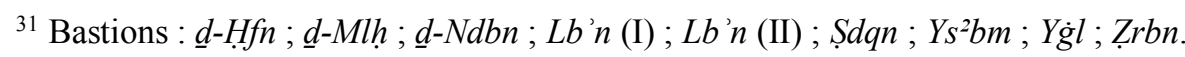

Courtines : $M \underline{d} b ; S^{2} b m t ; T n ' m ; T f s^{2}$.
} 
$Y s^{2} r$ et de ses fils $H f n m$ Rym et $W q h^{\prime} l$ Nbt (courtines 'brm, $\underline{d}$-Hs $b r$ et $H r s^{2}$; bastions $\underline{d}$-Mlh, Lb'n (I), Rbqn (I) et (II), Rdwwn, Zbyn et Zrbn) et des travaux hydrauliques dans le secteur irrigué de Malāḥā.

Au début du $1^{\text {er }}$ siècle av. J.-C., les commémorations de réalisations monumentales se font rares. Des réfections commanditées par le souverain lui-même, $Y \underline{t}^{\prime \prime} l \underline{S} d q$, sont entreprises sur le bastion $Y s^{2} b m$. L'achat de la résidence $M h d ̣ r$ est évoqué sans qu'il soit possible de préciser s'il s'agit ou non d'une construction neuve.

Enfin, des derniers travaux de réfection sont menés sur le rempart à la suite du passage de l'expédition romaine d' Ælius Gallus, dans le dernier quart du $1^{\text {er }}$ siècle av. J.-C.

En résumé, la monumentalisation de Barāqish intervient à partir du $6^{\mathrm{e}}$ siècle et surtout au cours du $5^{\mathrm{e}}-4^{\mathrm{e}}$ siècle av. J.-C. Au-delà de cette date, ce sont essentiellement des travaux d'entretien et de restaurations que l'on commémore.

\subsubsection{Le processus de nomination toponymique}

La plupart des monuments de Barāqish étaient désignés par un nom propre. Les raisons qui président au choix de tel ou tel nom ne peuvent être élucidées.

On observe de manière exceptionnelle que certaines structures sont nommées d'après le nom de la fraction de clan qui en finance la construction. C'est le cas du bastion $\underline{d}-H f f n$, financé par le sous-clan $H f n$, fraction de $G b^{\prime} n$. Le bastion $\underline{d}$-Mlh porte lui aussi le nom d'une fraction de clan qui n'est toutefois pas celle de ses bâtisseurs ( $Y f^{\prime} n$ fraction du clan $G b^{\prime} n$ ).

Ce processus de nomination reste donc occasionnel.

On observe également à de rares occasions des structures portant le nom des villes avec lesquelles les caravaniers de Yathill étaient en contact régulier : le bastion Zrbn est homonyme de la ville de Zrbn (auj. al-Ukhdūd, région de Najrān) ; la courtine Ddn est homonyme de la ville de $D d n$ (auj. al-'Ulā). $D d n$ étant par ailleurs un nom propre fréquemment attesté en Arabie du Sud, il est là encore difficile d'établir une règle.

\subsubsection{Par qui ?}

Il est remarquable que le roi de Ma'īn n'intervienne que rarement en tant que commanditaire, tant dans la capitale Qarnā (Ma'īn 2, 82, 90) qu'à Yathill où seules cinq inscriptions de construction sur 52 ont pour auteur le roi (Shaqab 18, B-M 6, B-M 103, B-M 249, B-M 259). La commande architecturale est avant tout le fait d'une élite locale relevant de cinq clans ${ }^{32}$ :

- ' 'ly'l (fraction $\left.S^{1} y l\right): 1$ ouvrage hydraulique à Malāhā ;

- $\quad$ Qrn (fraction $H \underline{H} k k t): 1$ ouvrage hydraulique à Darb aṣ-Ṣabī ;

- Ylqz (fraction Dbr) : 1 réalisation (aménagement intérieur du temple de Nakrah à Barāqish) ;

- $\quad M w q h$ (fractions $\dot{G r b t}$ et Zyrn) : 5 ouvrages de types variés (résidence, bastion, courtine, portique de temple, bassin) à Barāqish, Shaqab al-Manașșa et Darb aș-Ṣabī ;

\footnotetext{
${ }^{32}$ Les noms de clans sont rarement mentionnés dans les inscriptions, c'est le plus souvent la fraction de clan (sousclan) qui apparaît. Les noms des clans sont restitués grâce à la liste des Hiérodules de Ma īn qui associe fraction de clan et clan. Ajoutons que pour 21 des 52 inscriptions de construction, le clan n'a pu être restitué : 11 sont fragmentaires et l'information est perdue, 10 mentionnent un nom de sous-clan auquel aucun clan ne peut être associé au regard de la documentation actuelle - le détail est présenté en Annexe 2.
} 
- $\quad G b^{\prime} n$ (fractions Blh, Hfd, Hfn, $S^{2} \underline{t} m, Y f^{\prime} n, Z l w m n$ ) : 33 ouvrages parmi lesquels la quasitotalité des bastions et courtines ainsi que le temple de Wadd et les principaux aménagements intérieurs du temple de Nakrah.

Si les trois premiers clans interviennent de manière anecdotique (ouvrages hydrauliques périphériques, aménagements intérieurs de temple), c'est essentiellement le clan $G b^{\prime} n$ et dans une moindre mesure le clan $M w q h$ qui œuvrent à la monumentalisation du site.

Ces deux clans constituent indéniablement l'élite tribale marchande du royaume.

La suprématie du clan $G b^{\prime} n$ au sein du royaume se manifeste par plusieurs éléments :

- Il est l'un des plus anciens clans du royaume de Ma ${ }^{\mathrm{i}} \mathrm{in}$, attesté dès le $8^{\mathrm{e}}$ siècle av. J.-C., et des rois de Ma'īn sont issus de ce clan (Arbach \& Rossi 2012);

- Des membres de ce clan prennent pour épouse des femmes originaires des principales régions partenaires du commerce caravanier: Dadān (al-'Ulā), Ghazzat (Gaza), Hagar/Gerrha (al-Ḥufūf), Yathrib (Médine), Awsān (wādī Markha) (Ma`īn 93, Ma`īn 95, al-Saîd 2009) ;

- L'un d'eux commerce avec l'Égypte (Mșr), l'Assyrie ("s $\left.{ }^{2} r\right)$ et Ghazzat ( $\dot{G} z t$, Gaza) (Ma'in 7).

- Plusieurs membres occupent des postes honorifiques : chef des serviteurs du temple (?) ( $q d m$ 'hl 'mnhtn), chef des Minéens $\left(k b r M^{\prime} n\right)$ dans le comptoir de Dadān en Arabie du Nord (RÉS 3346, Ja 2288).

- On les trouve attestés à Madā’ in Șāliḥ (RÉS 3708) et al- 'Ulā (RÉS 3344, RÉS 3353) dans le Nord de l'Arabie ainsi que dans la capitale qatabānite Tamna' (CSAI I, 72).

- Ce clan finance la construction d'une large partie du rempart de Qarnā (Ma'īn) (Ma ${ }^{c} \bar{n} n$ 1, Ma'īn 7).

- On lui connaît enfin de vastes domaines fonciers autour de Qarnā (Ma ${ }^{` i ̄ n ~ 1) ~ e t ~ d e ~ Y a t h i l l, ~}$ jusqu'aux limites de la ville d'Inabba' (A-20-849) ${ }^{33}$.

Le clan $M w q h$ relève dans une moindre mesure de cette classe dominante minéenne :

- Mention de ses membres dans des inscriptions trouvées en Arabie du Nord, à al- 'Ulā (M 390, RÉS 3697, RÉS 3283) et Madā̄in Șāliḥ (RÉS 3708);

- Des membres de ce clan prennent pour épouse des femmes étrangères originaires d’Égypte (Ma`īn 93, Ma`īn 95) et de Khasham (lieu non identifié, Ma`īn 93).

- Le sarcophage d'un membre de ce clan enfin a été découvert dans l'oasis du Fayyoum en Égypte (RÉS 3427).

En résumé, on observe que Yathill, une fois intégrée au royaume de Ma īn ( $7^{\mathrm{e}}$ siècle av. J.-C.) ne fait pas l'objet d'un investissement monumental immédiatement. Ce n'est qu'au cours du $6^{\mathrm{e}}$ siècle que l'impulsion est donnée par des Minéens établis dans la capitale Qarnā (Ma'īn 6). Les fractions locales des clans dominants $M w q h$ et $G b^{\prime} n$ prennent alors le relais, sans que l'influence de la volonté royale ne puisse être déterminée. Yathill fait l'objet d'aménagements ambitieux bâtis entre le $6^{\mathrm{e}}$ et le $4^{\mathrm{e}}$ siècle av. J.-C. L'élément déterminant dans cette monumentalisation de la ville est la richesse accumulée par les principaux clans à travers les réseaux commerciaux transarabiques. Une dîme sur les recettes commerciales est prélevée par les temples ( $\mathrm{Ma}^{\mathrm{i}} \mathrm{i}$ 7). A Yathill, la construction monumentale est une manière fréquemment

\footnotetext{
${ }^{33}$ Le sous-clan $\underline{d}-Z l w m n$ mentionné dans ce texte est une fraction de $G b$ 'n $(\mathrm{Ma}$ '̄n $93 \mathrm{C} / 3,19)$.
} 
employée pour s'acquitter de cette taxe due au temple de 'Athtar dhu-Qabḍ" (RÉS 2975, RÉS 3012, RÉS 3021, RÉS 3022, RÉS 3535). 


\section{Annexe 1 - Les inscriptions de Barāqish - Sigles et concordances}

\subsection{Barāqish}

\subsubsection{Intra muros (indéterminé)}

\begin{tabular}{|c|c|c|c|c|c|c|}
\hline $\begin{array}{l}\text { Antonini Stela } \\
2\end{array}$ & & & & & & Antonini $2005: 308-309$ \\
\hline \multicolumn{7}{|l|}{ B-Int 1} \\
\hline \multicolumn{7}{|l|}{ B-Int 2} \\
\hline \multicolumn{7}{|l|}{ B-Int 3} \\
\hline B-Int 4 & & & $\begin{array}{l}M \\
208\end{array}$ & & RÉS 2985 & Garbini 1974 \\
\hline \multicolumn{7}{|l|}{ B-Int 5} \\
\hline \multicolumn{7}{|l|}{ B-Int 6} \\
\hline \multicolumn{7}{|l|}{ B-Int 7} \\
\hline \multicolumn{7}{|l|}{ B-Int 8} \\
\hline \multicolumn{7}{|l|}{ B-Int 9} \\
\hline \multicolumn{7}{|l|}{ B-Int 11} \\
\hline \multicolumn{7}{|l|}{ B-Int 12} \\
\hline \multicolumn{7}{|l|}{ B-Int 13} \\
\hline \multicolumn{7}{|l|}{ B-Int 14} \\
\hline \multicolumn{7}{|l|}{ B-Int 15} \\
\hline B-Int 16 & & & & & & Garbini 1974 \\
\hline \multicolumn{7}{|l|}{ B-Int 17} \\
\hline B-Int 18 & & & $\begin{array}{l}\text { M } \\
204\end{array}$ & & RÉS 2981 & Garbini 1974 \\
\hline B-Int 19 & $\begin{array}{l}\mathrm{Gr} \\
327\end{array}$ & $\begin{array}{l}\text { Hal } \\
489\end{array}$ & $\begin{array}{l}M \\
207\end{array}$ & $\begin{array}{l}\text { Nāmī B } \\
97\end{array}$ & RÉS 2984 & Bauer \& Lundin 1998 : 123 ; Garbini 1974 \\
\hline \multicolumn{7}{|l|}{ B-Int 20} \\
\hline B-Int 21 & & & $\begin{array}{l}M \\
206\end{array}$ & & RÉS 2983 & Garbini 1974 \\
\hline \multicolumn{7}{|l|}{ B-Int 22} \\
\hline \multicolumn{7}{|l|}{ B-Int 23} \\
\hline \multicolumn{7}{|l|}{ B-Int 24} \\
\hline \multicolumn{7}{|l|}{ B-Int 25} \\
\hline \multicolumn{7}{|l|}{ B-Int 26} \\
\hline \multicolumn{7}{|l|}{ B-Int 27} \\
\hline \multicolumn{7}{|l|}{ B-Int 28} \\
\hline \multicolumn{7}{|l|}{ B-Int 29} \\
\hline B-Int 30 & & & & & & \\
\hline B-Int 31 & & & & & & \\
\hline B-Int 32 & & & & & & \\
\hline B-Int 33 & & & & & & \\
\hline B-Int 34 & & & & & & \\
\hline B-Int 35 & & & & & & \\
\hline B-Int 39 & & & & & & \\
\hline B-Int 40 & & & & & & \\
\hline B-Int 41 & & & & & & \\
\hline B-Int 42 & & & & & & \\
\hline B-Int 45 & & & & & & \\
\hline B-Int 45 bis & & & & & & \\
\hline B-Int 46 & & & & & & \\
\hline B-Int 49 & & & & & & \\
\hline & $\begin{array}{l}\mathrm{Gr} \\
322\end{array}$ & & & & & Bauer \& Lundin $1998: 121$ \\
\hline Y.90.B 1 & & & & & & \\
\hline Y.90.B 2 & & & & & & \\
\hline
\end{tabular}




\begin{tabular}{|l|l|l|l|l|l|}
\hline Y.90.B 3 & & & & \\
\hline Y.90.B 4 & & & & \\
\hline Y.90.B 5 & & & & \\
\hline Y.90.B 6 & & & & \\
\hline Y.90.B 7 & & & & \\
\hline Y.90.B.ext 3 & & & & \\
\hline Y.90.B.ext 4 & & & & \\
\hline Y.90.B.ext 5 & & & & \\
\hline Y.90.B.ext 6 & & & & & \\
\hline Y.90.B.ext 7 & & & & & \\
\hline
\end{tabular}

6.1.2 Temple de Nakrah

\begin{tabular}{|c|c|c|c|c|c|}
\hline & & $\begin{array}{l}\text { Hal } \\
485\end{array}$ & $\begin{array}{l}M \\
203\end{array}$ & $R E ́ S 2980$ bis & $\begin{array}{l}\text { Garbini 1974; De Maigret \& Robin } \\
1993: 488-9\end{array}$ \\
\hline Y.03.B.A.1 & & & & & Agostini $2012: 6-7$ \\
\hline \multicolumn{6}{|l|}{ Y.90.B.A 1} \\
\hline \multicolumn{6}{|l|}{ Y.90.B.A 2} \\
\hline Y.90.B.A 3 & & & & & De Maigret \& Robin $1993: 480$ \\
\hline \multicolumn{6}{|l|}{ Y.90.B.A 4} \\
\hline \multicolumn{6}{|l|}{ Y.90.B.A 5} \\
\hline \multicolumn{6}{|l|}{ Y.90.B.A 6} \\
\hline Y.90.B.A 7 & & & & & De Maigret \& Robin 1993 : 480-481 \\
\hline \multicolumn{6}{|l|}{ Y.90.B.A 8} \\
\hline \multicolumn{6}{|l|}{ Y.90.B.A 9} \\
\hline \multicolumn{6}{|l|}{ Y.90.B.A $10(A+B)$} \\
\hline \multicolumn{6}{|l|}{ Y.90.B.A 11} \\
\hline \multicolumn{6}{|l|}{ Y.90.B.A 12} \\
\hline \multicolumn{6}{|l|}{ Y.90.B.A 13} \\
\hline \multicolumn{6}{|l|}{ Y.90.B.A $14(\mathrm{~A}+\mathrm{B})$} \\
\hline \multicolumn{6}{|c|}{ Y.90.B.A 15} \\
\hline \multicolumn{6}{|c|}{$\begin{array}{l}\text { Y.90.B.A } 16 \quad(+ \\
\text { Y.86.B.ext 1) }\end{array}$} \\
\hline \multicolumn{6}{|c|}{ Y.90.B.A 17} \\
\hline Y.90.B.A 18 & & & & & De Maigret \& Robin $1993: 482$ \\
\hline \multicolumn{6}{|l|}{ Y.90.B.A 19} \\
\hline \multicolumn{6}{|l|}{ Y.90.B.ext 1} \\
\hline Y.90.B.ext 2 & $\begin{array}{l}\text { YM } \\
26117\end{array}$ & & & & De Maigret \& Robin $1993: 482-483$ \\
\hline \multicolumn{6}{|l|}{ Y.92.B.A 1} \\
\hline \multicolumn{6}{|l|}{ Y.92.B.A 2} \\
\hline \multicolumn{6}{|l|}{ Y.92.B.A 3} \\
\hline \multicolumn{6}{|l|}{ Y.92.B.A 4} \\
\hline \multicolumn{6}{|l|}{ Y.92.B.A 6} \\
\hline \multicolumn{6}{|l|}{ Y.92.B.A 7} \\
\hline \multicolumn{6}{|l|}{ Y.92.B.A 8} \\
\hline \multicolumn{6}{|l|}{ Y.92.B.A 9} \\
\hline Y.92.B.A 10 & & & & & \\
\hline Y.92.B.A 11 & & & & & \\
\hline Y.92.B.A 12 & & & & & \\
\hline Y.92.B.A 13 & & & & & \\
\hline Y.92.B.A 14 & & & & & \\
\hline Y.92.B.A 15 & & & & & De Maigret \& Robin 1993 : 483-484 \\
\hline Y.92.B.A 16 & & & & & \\
\hline Y.92.B.A 17 & & & & & \\
\hline Y.92.B.A 18 & & & & & \\
\hline Y.92.B.A 19 & & & & & \\
\hline Y.92.B.A 20 & & & & & De Maigret \& Robin $1993: 484-485$ \\
\hline Y.92.B.A $21+30$ & & & & & De Maigret \& Robin 1993 : 485-486 \\
\hline
\end{tabular}




\begin{tabular}{|c|c|}
\hline \multicolumn{2}{|l|}{ Y.92.B.A 22} \\
\hline \multicolumn{2}{|l|}{ Y.92.B.A 23} \\
\hline \multicolumn{2}{|l|}{ Y.92.B.A 24} \\
\hline \multicolumn{2}{|l|}{ Y.92.B.A 25} \\
\hline \multicolumn{2}{|l|}{ Y.92.B.A 26} \\
\hline \multicolumn{2}{|l|}{ Y.92.B.A 27} \\
\hline Y.92.B.A 28 & De Maigret \& Robin 1993 : 485-486 \\
\hline Y.92.B.A 29 & Gnoli $1996,1145-1159$ \\
\hline \multicolumn{2}{|l|}{ Y.92.B.A 31} \\
\hline Y.92.B.A $32+5$ & De Maigret \& Robin 1993 : 483, 487 \\
\hline \multicolumn{2}{|l|}{ Y.92.B.A 33} \\
\hline \multicolumn{2}{|l|}{ Y.92.B.A 34} \\
\hline \multicolumn{2}{|l|}{ Y.92.B.A 35} \\
\hline \multicolumn{2}{|l|}{ Y.92.B.A 36} \\
\hline \multicolumn{2}{|l|}{ Y.92.B.A 37 A-B } \\
\hline Y.92.B.A 38 & De Maigret \& Robin 1993 : 462 \\
\hline Y.92.B.A 39 & De Maigret \& Robin 1993 : 463 \\
\hline Y.92.B.A 40 & De Maigret \& Robin 1993 : 464 \\
\hline \multicolumn{2}{|l|}{ Y.92.B.A 41} \\
\hline \multicolumn{2}{|l|}{ Y.92.B.A 42} \\
\hline \multicolumn{2}{|l|}{ Y.92.B.A 43} \\
\hline \multicolumn{2}{|l|}{ Y.92.B.A 44} \\
\hline \multicolumn{2}{|l|}{ Y.92.B.A 45} \\
\hline Y.92.B.A 46 & De Maigret \& Robin 1993 : 487 \\
\hline \multicolumn{2}{|l|}{ Y.92.B.A 47} \\
\hline \multicolumn{2}{|l|}{ Y.92.B.A 48} \\
\hline \multicolumn{2}{|l|}{ Y.92.B.A 49} \\
\hline \multicolumn{2}{|l|}{ Y.92.B.A 50} \\
\hline \multicolumn{2}{|l|}{ Y.92.B.A 51} \\
\hline \multicolumn{2}{|l|}{ Y.92.B.A 52} \\
\hline \multicolumn{2}{|l|}{ Y.92.B.A 53} \\
\hline \multicolumn{2}{|l|}{ Y.92.B.A 54} \\
\hline Y.92.B.A 55 & \\
\hline
\end{tabular}

6.1.3 Temple de 'Athtar dhu-Qabd

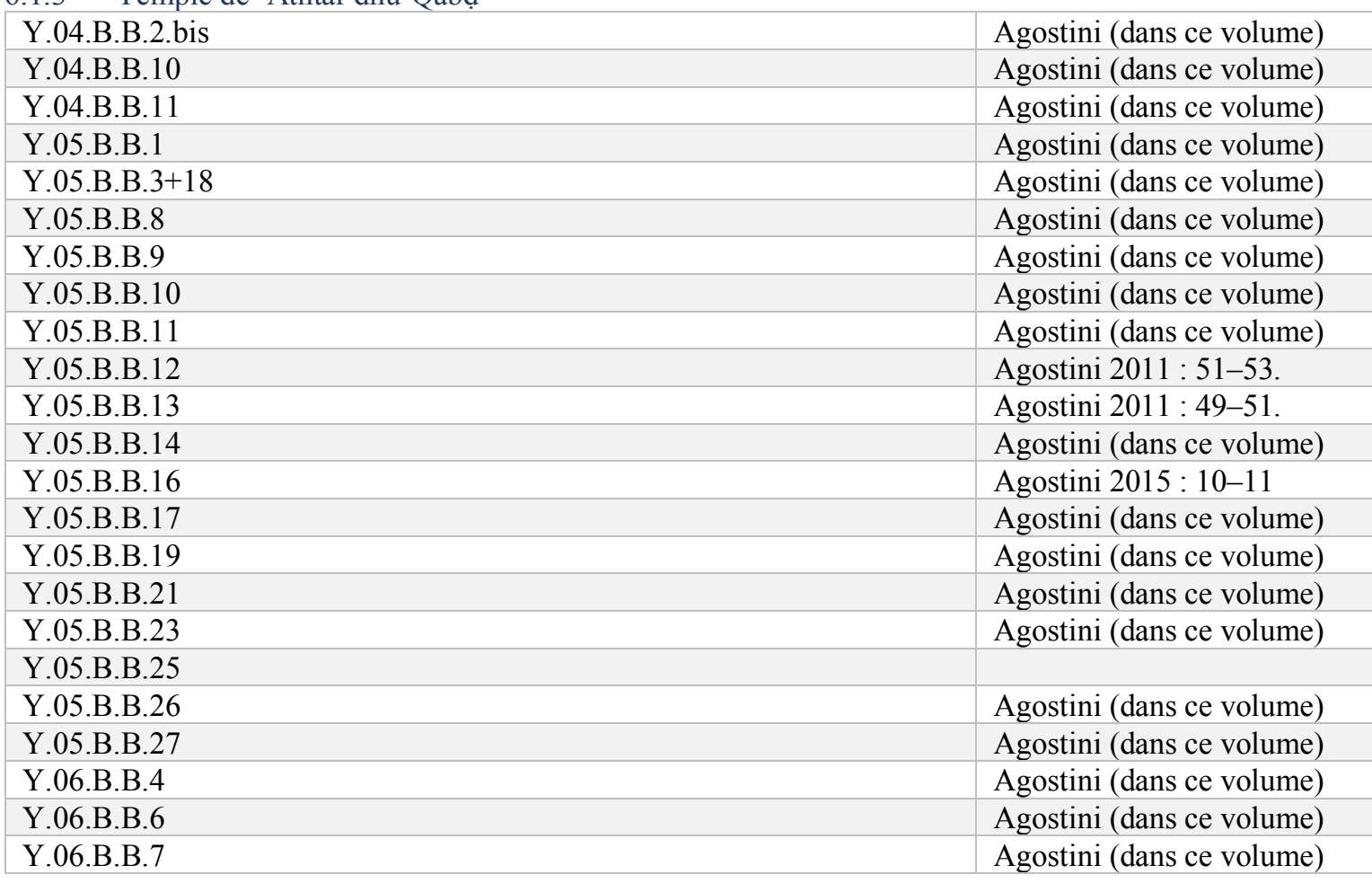


Y.06.B.B.8

Y.06.B.B. 10

Y.06.B.B. 11
Agostini (dans ce volume)

Agostini (dans ce volume) Agostini (dans ce volume)

\subsubsection{Rempart}

\begin{tabular}{|c|c|c|c|c|c|c|}
\hline B-Int 36 & & & & & & \\
\hline B-Int 37 & & & & & & \\
\hline B-Int 38 & & & & & & \\
\hline B-Int 43 & & & & & & \\
\hline B-Int 44 & & & & & & \\
\hline B-Int 48 & & & & & & \\
\hline B-Int 50 & & & & & & \\
\hline B-M 1 & Gr 316 & $\begin{array}{l}\text { Hal } 478 \\
+479\end{array}$ & M 197 & Nāmī B 63 & $R E ́ S 2975$ & $\begin{array}{l}\text { Bauer \& Lundin 1998: 118- } \\
120 ; \text { Garbini 1974; Müller } \\
1982: 132-133\end{array}$ \\
\hline B-M 2 & & & & & & \\
\hline B-M 3 & & & & & & \\
\hline B-M 4 & Gr 321 & Hal 540 & M 252 & & RÉS 3027 & $\begin{array}{l}\text { Bauer \& Lundin 1998: } 121 \text {; } \\
\text { Garbini } 1974\end{array}$ \\
\hline B-M 5 & Gr 319 & Hal 539 & M 251 & & $R E ́ S 3026$ & $\begin{array}{l}\text { Bauer \& Lundin 1998: } 121 \text {; } \\
\text { Garbini } 1974\end{array}$ \\
\hline B-M 6 & Gr 318 & Hal 538 & M 250 & Nāmī B 64 & $R E ́ S 3025$ & $\begin{array}{l}\text { Bauer \& Lundin 1998: } 120 \text {; } \\
\text { Garbini } 1974\end{array}$ \\
\hline B-M 7 & & & M 249 & & $R E ́ S 3024$ & Garbini 1974 \\
\hline B-M 8 & & & & & & \\
\hline B-M 9 & & & & & & \\
\hline B-M 10 & & Hal 520 & M 236 & & RÉS 3012 & $\begin{array}{l}\text { Robin 1979a : 103; Garbini } \\
1974\end{array}$ \\
\hline B-M 11 & & & & & & \\
\hline B-M 12 & & & M 237 & $R E ́ S 3013$ & & Garbini 1974 \\
\hline B-M 13 & & & M 238 & & $R E ́ S 3014$ & Garbini 1974 \\
\hline B-M 14 & & & & & & \\
\hline B-M 15 & & & M 255 & & RÉS 3029 & Garbini 1974 \\
\hline B-M 16 & & & M 256 & & $R E ́ S 3030$ & Garbini 1974 \\
\hline B-M 17 & & & & & & \\
\hline B-M 18 & & & M 258 & & $R E ́ S 3032$ & Garbini 1974 \\
\hline B-M 19 & & & & & & \\
\hline B-M 19 bis & & & M 257 & & $R E ́ S 3031$ & Garbini 1974 \\
\hline B-M 20 & & & & & & \\
\hline B-M 21 & & & M 248 & & $R E ́ S 3023$ & Garbini 1974 \\
\hline B-M 22 & & & & & & \\
\hline B-M 23 & & & M 253 & & $R E ́ S 3028$ & Garbini 1974 \\
\hline B-M 23 bis & & & & & & \\
\hline B-M 24 & & & & & & \\
\hline B-M 25 & & & M 260 & & RÉS 3034 & Garbini 1974 \\
\hline B-M 26 & & & M 254 & & $\begin{array}{l}R E S 3028 \\
\text { bis }\end{array}$ & Garbini 1974 \\
\hline B-M 27 & & & & & & \\
\hline B-M 28 & & & & & & \\
\hline B-M 29 & & & & & & \\
\hline B-M 30 & & & & & & \\
\hline B-M 31 & & & & & & \\
\hline B-M 32 & & & & & & \\
\hline B-M 33 & & & M 271 & & $R E ́ S 3045$ & Garbini 1974 \\
\hline B-M 34 & & & M 259 & & $R E ́ S 3033$ & Garbini 1974 \\
\hline B-M 35 & & & & & & \\
\hline B-M 36 & & & & & & \\
\hline B-M 37 & & & & & & \\
\hline
\end{tabular}




\begin{tabular}{|c|c|c|c|c|c|c|}
\hline B-M 38 & & & $\begin{array}{l}\text { M } 261+ \\
273\end{array}$ & & $\begin{array}{l}\text { RÉS } 3035 \\
+3048\end{array}$ & Garbini 1974 \\
\hline B-M 39 & & & M 272 & & RÉS 3047 & Garbini 1974 \\
\hline \multicolumn{7}{|l|}{ B-M 40} \\
\hline B-M 41 & & & M 264 & & RÉS 3038 & Garbini 1974 \\
\hline B-M 42 & Gr 277 & Hal 555 & M 267 & & RÉS 3041 & $\begin{array}{l}\text { Bauer \& Lundin 1998: } 102 \text {; } \\
\text { Garbini } 1974\end{array}$ \\
\hline B-M 43 & & & M 268 & & $R E ́ S 3042$ & Garbini 1974 \\
\hline B-M 44 & & & $\begin{array}{l}\text { M 266+ } \\
265+ \\
274\end{array}$ & & $\begin{array}{l}R E ́ S 3040 \\
+3039+ \\
3049\end{array}$ & Garbini 1974 \\
\hline \multicolumn{7}{|l|}{ B-M 45} \\
\hline B-M 46 & & & M 269 & & $R E ́ S 3043$ & Garbini 1974 \\
\hline \multicolumn{7}{|l|}{ B-M 47} \\
\hline \multicolumn{7}{|l|}{ B-M 48} \\
\hline \multicolumn{7}{|l|}{ B-M 49} \\
\hline \multicolumn{7}{|l|}{ B-M 50} \\
\hline \multicolumn{7}{|l|}{ B-M 51} \\
\hline \multicolumn{7}{|l|}{ B-M 52} \\
\hline \multicolumn{7}{|l|}{ B-M 53} \\
\hline \multicolumn{7}{|l|}{ B-M 54} \\
\hline B-M 55 & & & M 410 & Nami B. 74 & & Garbini 1974 \\
\hline $\begin{array}{l}\text { B-M } 56= \\
\text { Robin/Barāqish } \\
1\end{array}$ & & & M 283 & & RÉS 3060 & $\begin{array}{l}\text { Garbini } 1974 ; \text { Robin } 1979 a \text { : } \\
\text { 104-105 ; Robin 1979b : } 195\end{array}$ \\
\hline \multicolumn{7}{|l|}{ B-M 57} \\
\hline \multicolumn{7}{|l|}{ B-M 58} \\
\hline \multicolumn{7}{|l|}{ B-M 59} \\
\hline \multicolumn{7}{|l|}{ B-M 60} \\
\hline \multicolumn{7}{|l|}{ B-M 61} \\
\hline B-M 62 & & & M 280 & & $R E ́ S 3057$ & Garbini 1974 \\
\hline \multicolumn{7}{|l|}{ B-M 63} \\
\hline \multicolumn{7}{|l|}{ B-M 64} \\
\hline \multicolumn{7}{|l|}{ B-M 65} \\
\hline \multicolumn{7}{|l|}{ B-M 66} \\
\hline \multicolumn{7}{|l|}{ B-M 67} \\
\hline B-M 68 & & & M 415 & Nami B. 81 & & Garbini 1974 \\
\hline B-M 69 & & & M 414 & Nami B. 80 & & Garbini 1974 \\
\hline B-M 70 & & & M 413 & Nami B. 79 & & Garbini 1974 \\
\hline B-M 71 & & & M 412 & Nami B. 78 & & Garbini 1974 \\
\hline B-M 72 & & & M 411 & Nami B. 77 & & Garbini 1974 \\
\hline B-M 73 & & & $\begin{array}{l}\text { M 416 + } \\
275+ \\
275\end{array}$ & & $\begin{array}{l}R E S S 3053 \\
+3056+ \\
3050\end{array}$ & Garbini 1974 \\
\hline \multicolumn{7}{|l|}{ B-M 74} \\
\hline B-M 75 & & & M 278 & & $\begin{array}{l}R E ́ S 3052 \\
\mathrm{~B}\end{array}$ & Garbini 1974 \\
\hline B-M 76 & & & $\begin{array}{l}\text { M } 276+ \\
277\end{array}$ & & $\begin{array}{l}R E S S 3051 \\
+3052 \mathrm{a}\end{array}$ & Garbini 1974 \\
\hline \multicolumn{7}{|l|}{ B-M 77} \\
\hline B-M 78 & & & & & & \\
\hline B-M 79 & & & & & & \\
\hline B-M 80 & & & M 279 & & $R E ́ S 3054$ & Garbini 1974 \\
\hline B-M 81 & & & M 417 & Nami B. 85 & & Garbini 1974 \\
\hline B-M 82 & & & M 275 & & RÉS 3050 & Garbini 1974 \\
\hline B-M 83 & & & M 420 & Nami B. 91 & & Garbini 1974 \\
\hline B-M 84 & & & M 421 & Nami B. 93 & & Garbini 1974 \\
\hline B-M 85 & & & M 422 & Nami B. 95 & & Garbini 1974 \\
\hline
\end{tabular}




\begin{tabular}{|c|c|c|c|c|}
\hline B-M 86 & M 274A & & $R E ́ S 3055$ & Garbini 1974 \\
\hline B-M 87 & M 423 & Nami B. 96 & & Garbini 1974 \\
\hline \multicolumn{5}{|l|}{ B-M 88} \\
\hline B-M 89 & M 282 & & RÉS 3059 & Garbini 1974 \\
\hline \multicolumn{5}{|l|}{ B-M 90} \\
\hline \multicolumn{5}{|l|}{ B-M 91} \\
\hline B-M 92 & M 424 & Nami B. 99 & & Garbini 1974 \\
\hline \multicolumn{5}{|l|}{ B-M 93} \\
\hline B-M 94 & M 425 & $\begin{array}{l}\text { Nami } \\
100\end{array}$ & & Garbini 1974 \\
\hline \multicolumn{5}{|l|}{ B-M 95} \\
\hline B-M 96 & M 426 & $\begin{array}{l}\text { Nami B. } \\
101\end{array}$ & & Garbini 1974 \\
\hline \multicolumn{5}{|l|}{ B-M 97} \\
\hline \multicolumn{5}{|l|}{ B-M 98} \\
\hline \multicolumn{5}{|l|}{ B-M 99} \\
\hline \multicolumn{5}{|l|}{ B-M 100} \\
\hline \multicolumn{5}{|l|}{ B-M 101} \\
\hline \multicolumn{5}{|l|}{ B-M 102} \\
\hline $\begin{array}{l}\text { B-M } 103= \\
\text { Robin- } \\
\text { Baraqish } 80\end{array}$ & & & & Robin 1979b : 193-194 \\
\hline \multicolumn{5}{|l|}{ B-M 104} \\
\hline \multicolumn{5}{|l|}{ B-M 105} \\
\hline B-M 106 & M 427 & $\begin{array}{l}\text { Nami B. } \\
102\end{array}$ & & Garbini 1974 \\
\hline \multicolumn{5}{|l|}{ B-M 107} \\
\hline \multicolumn{5}{|l|}{ B-M 108} \\
\hline \multicolumn{5}{|l|}{ B-M 109} \\
\hline B-M 110 & M 428 & $\begin{array}{l}\text { Nami B. } \\
104\end{array}$ & & Garbini 1974 \\
\hline B-M 111 & $\begin{array}{l}\text { M } 431+ \\
432\end{array}$ & $\begin{array}{l}\text { Nami B. } \\
107+\text { Nami } \\
\text { B. } 108\end{array}$ & & Garbini 1974 \\
\hline \multicolumn{5}{|l|}{ B-M 112} \\
\hline \multicolumn{5}{|l|}{ B-M 113} \\
\hline B-M 114 & M 429 & $\begin{array}{l}\text { Nami B. } \\
105\end{array}$ & & Garbini 1974 \\
\hline B-M 115 & $\begin{array}{l}\text { M 436 + } \\
430\end{array}$ & $\begin{array}{l}\text { Nami B. } \\
112+\text { Nami } \\
\text { B. } 106\end{array}$ & & Garbini 1974 \\
\hline \multicolumn{5}{|l|}{ B-M 116} \\
\hline \multicolumn{5}{|l|}{ B-M 117} \\
\hline \multicolumn{5}{|l|}{ B-M 118} \\
\hline B-M 119 & M 434 & $\begin{array}{l}\text { Nami } \\
110\end{array}$ & & Garbini 1974 \\
\hline B-M 120 & M 433 & $\begin{array}{l}\text { Nami B. } \\
109\end{array}$ & & Garbini 1974 \\
\hline B-M 121 & M 437 & $\begin{array}{l}\text { Nami } \\
113\end{array}$ & & Garbini 1974 \\
\hline \multicolumn{5}{|l|}{ B-M 122} \\
\hline \multicolumn{5}{|l|}{ B-M 123} \\
\hline B-M 124 & M 435 & $\begin{array}{l}\text { Nami } \quad \text { B. } \\
111\end{array}$ & & Garbini 1974 \\
\hline \multicolumn{5}{|l|}{ B-M 125} \\
\hline B-M 126 & M 438 & $\begin{array}{l}\text { Nami } \\
114\end{array}$ & & Garbini 1974 \\
\hline B-M 127 & & & & \\
\hline
\end{tabular}




\begin{tabular}{|c|c|c|c|c|c|c|}
\hline \multicolumn{7}{|l|}{ B-M 128} \\
\hline \multicolumn{7}{|l|}{ B-M 129} \\
\hline \multicolumn{7}{|l|}{ B-M 130} \\
\hline \multicolumn{7}{|l|}{ B-M 131} \\
\hline B-M 132 & $\begin{array}{l}\text { Gr } 249 \\
+250\end{array}$ & Hal 494 & $\begin{array}{l}\text { M } 439+ \\
212\end{array}$ & $\begin{array}{l}\text { Nami B. } \\
116(+R E S \\
2989)\end{array}$ & & $\begin{array}{l}\text { Bauer \& Lundin 1998: } 118 \text {; } \\
\text { Garbini } 1974\end{array}$ \\
\hline \multicolumn{7}{|l|}{ B-M 133} \\
\hline B-M 134 & Gr 252 & & M 440 & $\begin{array}{l}\text { Nami } \quad \text { B. } \\
118\end{array}$ & & $\begin{array}{l}\text { Bauer \& Lundin 1998: } 118 \text {; } \\
\text { Garbini } 1974\end{array}$ \\
\hline B-M 135 & Gr 253 & & & & & Bauer \& Lundin $1998: 118$ \\
\hline B-M 136 & $\begin{array}{l}\text { Gr } 261 \\
+260+ \\
248 \quad+ \\
257\end{array}$ & $\begin{array}{l}\text { Hal } 501 \\
+500+ \\
493+ \\
497\end{array}$ & $\begin{array}{ll}\text { M 219 + } \\
218+ \\
211+ \\
215\end{array}$ & & $\begin{array}{l}R E S 2996 \\
+2995+ \\
2988+ \\
2992\end{array}$ & $\begin{array}{l}\text { Bauer \& Lundin 1998: } 119 \text {; } \\
\text { Garbini } 1974\end{array}$ \\
\hline \multicolumn{7}{|l|}{ B-M 137} \\
\hline B-M 138 & Gr 255 & Hal 495 & M 213 & Nāmī B 119 & RÉS 2990 & $\begin{array}{l}\text { Bauer \& Lundin 1998: } 118 \text {; } \\
\text { Garbini } 1974\end{array}$ \\
\hline B-M 139 & $\begin{array}{lr}\text { Gr } & 256 \\
+ & \text { Gr } \\
259 \quad+ \\
\text { Nāmī } & \text { B } \\
109 \quad+ \\
\text { Gr } 258\end{array}$ & $\begin{array}{l}\text { Hal } 496 \\
+498+ \\
499\end{array}$ & $\begin{array}{l}\text { M 214+ } \\
216+ \\
217\end{array}$ & & $\begin{array}{l}\text { RES } 2991 \\
+2993+ \\
2994\end{array}$ & $\begin{array}{l}\text { Bauer \& Lundin 1998: 91; } \\
\text { Garbini } 1974\end{array}$ \\
\hline B-M 141 & Gr 262 & Hal 502 & M 220 & & RÉS 2997 & $\begin{array}{l}\text { Bauer \& Lundin 1998: 92; } \\
\text { Garbini 1974 }\end{array}$ \\
\hline \multicolumn{7}{|l|}{ B-M 142} \\
\hline \multicolumn{7}{|l|}{ B-M 143} \\
\hline \multicolumn{7}{|l|}{ B-M 144} \\
\hline B-M 145 & Gr 264 & & M 441 & $\begin{array}{l}\text { Nami } \quad \text { B. } \\
126\end{array}$ & & $\begin{array}{l}\text { Bauer \& Lundin 1998: 92; } \\
\text { Garbini 1974 }\end{array}$ \\
\hline \multicolumn{7}{|l|}{ B-M 146} \\
\hline B-M 147 & Gr 271 & Hal 504 & M 222 & Nāmī B 127 & RÉS 2999 & $\begin{array}{l}\text { Bauer \& Lundin } 1998 \text { : 95-98; } \\
\text { Garbini } 1974 \text {; Robin }\end{array}$ \\
\hline B-M 148 & Gr 270 & Hal 508 & M 225 & Nāmī B 131 & $R E ́ S 3002$ & $\begin{array}{l}\text { Bauer \& Lundin } 1998 \text { : 94-95; } \\
\text { Garbini } 1974\end{array}$ \\
\hline B-M 149 & Gr 268 & Hal 507 & M 224 & Nāmī B 129 & RÉS 3001 & $\begin{array}{l}\text { Bauer \& Lundin 1998: 93; } \\
\text { Garbini } 1974\end{array}$ \\
\hline B-M 150 & $\begin{array}{lr}\mathrm{Gr} & 267 \\
+ & \mathrm{Gr} \\
266 & \end{array}$ & $\begin{array}{l}\text { Hal } 506 \\
+505\end{array}$ & M 223 & Nāmī B 128 & RÉS 3000 & $\begin{array}{l}\text { Bauer \& Lundin 1998: 92; } \\
\text { Garbini } 1974\end{array}$ \\
\hline B-M 151 & Gr 269 & & M 442 & $\begin{array}{l}\text { Nami } \quad \text { B. } \\
130\end{array}$ & & $\begin{array}{l}\text { Bauer \& Lundin } 1998 \text { : 93-94; } \\
\text { Garbini } 1974\end{array}$ \\
\hline \multicolumn{7}{|l|}{ B-M 152} \\
\hline \multicolumn{7}{|l|}{ B-M 153} \\
\hline \multicolumn{7}{|l|}{ B-M 154} \\
\hline \multicolumn{7}{|l|}{ B-M 155} \\
\hline B-M 156 & & & M 160 & & RÉS 2938 & Garbini 1974 \\
\hline B-M 157 & & & M 158 & & RES 2936 & Garbini 1974 \\
\hline B-M 158 & & & M 157 & & RÉS 2935 & Garbini 1974 \\
\hline B-M 159 & & & M 156 & & RÉS 2934 & Garbini 1974 \\
\hline B-M 160 & & & M 155 & & RÉS 2933 & Garbini 1974 \\
\hline B-M 161 & & & M 154 & & RÉS 2932 & Garbini 1974 \\
\hline B-M 162 & & & M 153 & & RÉS 2931 & Garbini 1974 \\
\hline B-M 163 & & & M 152 & & RÉS 2930 & Garbini 1974 \\
\hline \multicolumn{7}{|l|}{ B-M 164} \\
\hline \multicolumn{7}{|l|}{ B-M 165} \\
\hline B-M 166 & & & & & & \\
\hline
\end{tabular}




\begin{tabular}{|c|c|c|c|c|c|c|}
\hline \multicolumn{7}{|l|}{ B-M 167} \\
\hline \multicolumn{7}{|l|}{ B-M 168} \\
\hline \multicolumn{7}{|l|}{ B-M 169} \\
\hline \multicolumn{7}{|l|}{ B-M 171} \\
\hline \multicolumn{7}{|l|}{ B-M 172} \\
\hline \multicolumn{7}{|l|}{ B-M 173} \\
\hline \multicolumn{7}{|l|}{ B-M 174} \\
\hline \multicolumn{7}{|l|}{ B-M 175} \\
\hline B-M 176 & Gr 274 & & & & & Bauer \& Lundin 1998 : 102 \\
\hline \multicolumn{7}{|l|}{ B-M 177} \\
\hline B-M 178 & Gr 275 & Hal 442 & M 166 & Nāmī B 139 & RÉS 2944 & $\begin{array}{l}\text { Bauer \& Lundin 1998: } 102 \text {; } \\
\text { Garbini } 1974\end{array}$ \\
\hline B-M 179 & & Hal 441 & M 165 & & RÉS 2943 & Garbini 1974 \\
\hline B-M 180 & & & M 164 & & RÉS 2942 & Garbini 1974 \\
\hline \multicolumn{7}{|l|}{ B-M 181} \\
\hline B-M 182 & $\begin{array}{l}\text { Gr } 272 \\
+273\end{array}$ & $\begin{array}{l}\text { Hal } 424 \\
+435\end{array}$ & $\begin{array}{l}M \\
151+163\end{array}$ & & $\begin{array}{l}R E S S 2929 \\
+2941+ \\
2945+ \\
2946\end{array}$ & $\begin{array}{l}\text { Bauer \& Lundin 1998: } 98 \text {; } \\
\text { Garbini 1974; Robin 1979a: } \\
104\end{array}$ \\
\hline \multicolumn{7}{|l|}{ B-M 183} \\
\hline \multicolumn{7}{|l|}{ B-M 184} \\
\hline \multicolumn{7}{|l|}{ B-M 185} \\
\hline \multicolumn{7}{|l|}{ B-M 186} \\
\hline B-M 187 & Gr 278 & & & & & Bauer \& Lundin $1998: 102$ \\
\hline \multicolumn{7}{|l|}{ B-M 188} \\
\hline \multicolumn{7}{|l|}{ B-M 189} \\
\hline B-M 190 & Gr 282 & & М 444 & $\begin{array}{l}\text { Nami B. } \\
143\end{array}$ & & $\begin{array}{l}\text { Bauer \& Lundin 1998: 104- } \\
105 \text {; Garbini } 1974\end{array}$ \\
\hline B-M 191 & Gr 279 & & M 467 & & & $\begin{array}{l}\text { Bauer \& Lundin 1998: } 102 \text {; } \\
\text { Garbini } 1974\end{array}$ \\
\hline \multicolumn{7}{|l|}{ B-M 192} \\
\hline \multicolumn{7}{|l|}{ B-M 193} \\
\hline B-M 194 & Gr 284 & Hal 445 & M 167 & Nāmī B 145 & $R E ́ S 2947$ & $\begin{array}{l}\text { Bauer \& Lundin 1998: } 107 \text {; } \\
\text { Garbini } 1974\end{array}$ \\
\hline \multicolumn{7}{|l|}{ B-M 195} \\
\hline B-M 196 & $\begin{array}{l}\text { Gr } 285 \\
+283\end{array}$ & $\begin{array}{l}\text { Hal } 447 \\
+446\end{array}$ & M 168 & Nāmī B 146 & $R E ́ S 2948$ & $\begin{array}{l}\text { Bauer \& Lundin 1998: 105- } \\
107 \text {; Garbini } 1974\end{array}$ \\
\hline \multicolumn{7}{|l|}{ B-M 197} \\
\hline B-M 198 & & & M 443 & $\begin{array}{l}\text { Nami } \quad \text { B. } \\
138\end{array}$ & & Garbini 1974 \\
\hline B-M 199 & Gr 287 & & & & & Bauer \& Lundin $1998: 107$ \\
\hline \multicolumn{7}{|l|}{ B-M 200} \\
\hline \multicolumn{7}{|l|}{ B-M 201} \\
\hline B-M 202 & Gr 288 & & M 446 & $\begin{array}{l}\text { Nami } \quad \text { B. } \\
147\end{array}$ & & $\begin{array}{l}\text { Bauer \& Lundin 1998: } 107 \text {; } \\
\text { Garbini } 1974\end{array}$ \\
\hline B-M 203 & Gr 290 & Hal 449 & M 170 & Nāmī B 149 & RÉS 2950 & $\begin{array}{l}\text { Bauer \& Lundin 1998: } 108 \text {; } \\
\text { Garbini } 1974\end{array}$ \\
\hline $\begin{array}{l}{[\mathrm{B}-\mathrm{M} 211+] \mathrm{B}-} \\
\text { M } 204\end{array}$ & Gr 289 & Hal 448 & M 169 & Nāmī B 148 & RÉS 2949 & $\begin{array}{l}\text { Bauer \& Lundin 1998: 107- } \\
\text { 108; Garbini } 1974\end{array}$ \\
\hline \multicolumn{7}{|l|}{ B-M 205} \\
\hline B-M 206 & & & M 173 & & $R E ́ S 2953$ & Garbini 1974 \\
\hline B-M 207 & & & M 171 & & RÉS 2951 & Garbini 1974 \\
\hline B-M 208 & & & & & & \\
\hline B-M 209 & & & & & & \\
\hline B-M 210 & & & M 174 & & $R E ́ S 2954$ & Garbini 1974 \\
\hline $\begin{array}{l}\text { B-M 211 [+ B- } \\
\text { M 204] }\end{array}$ & & & M 172 & & RÉS 2952 & $\begin{array}{l}\text { Garbini 1974; Müller 1982: } \\
132\end{array}$ \\
\hline B-M 211 bis & & & M 177 & & RÉS 2957 & \\
\hline
\end{tabular}




\begin{tabular}{|c|c|c|c|c|c|c|}
\hline B-M 212 & & & M 178 & & $R E ́ S 2958$ & Garbini 1974 \\
\hline \multicolumn{7}{|l|}{ B-M 213} \\
\hline \multicolumn{7}{|l|}{ B-M 214} \\
\hline \multicolumn{7}{|l|}{ B-M 215} \\
\hline B-M 216 & Gr 291 & & M 179 & & RÉS 2959 & $\begin{array}{l}\text { Bauer \& Lundin 1998: } 108 \text {; } \\
\text { Garbini } 1974\end{array}$ \\
\hline \multicolumn{7}{|l|}{ B-M 217} \\
\hline $\begin{array}{l}\text { B-M } 218= \\
\text { Y.03.B.R44- } \\
45.4\end{array}$ & & & M 180 & & RÉS 2960 & $\begin{array}{l}\text { Garbini } 1974 \text {; Agostini dans ce } \\
\text { volume }\end{array}$ \\
\hline \multicolumn{7}{|l|}{ B-M 219} \\
\hline B-M 220 & & & M 181 & & RÉS 2961 & Garbini 1974 \\
\hline B-M 221 & & & M 226 & & RÉS 3003 & Garbini 1974 \\
\hline B-M 222 & Gr 293 & Hal 512 & M 228 & Nāmī B 26 & RÉS 3005 & $\begin{array}{l}\text { Bauer \& Lundin 1998: } 109 \text {; } \\
\text { Garbini } 1974\end{array}$ \\
\hline B-M 223 & Gr 292 & Hal 511 & M 227 & Nāmī B 23 & RÉS 3004 & $\begin{array}{l}\text { Bauer \& Lundin 1998: 108- } \\
109 \text {; Garbini } 1974\end{array}$ \\
\hline $\begin{array}{l}\text { B-M } 224 \\
\text { [frag. de Bauer } \\
5=\text { B-M } 231+ \\
224+238]\end{array}$ & & Hal 462 & M 182 & & RÉS 2962 & $\begin{array}{l}\text { Bauer \& Lundin 1998: 110- } \\
112 \text {; Garbini } 1974\end{array}$ \\
\hline \multicolumn{7}{|l|}{ B-M 225} \\
\hline B-M 226 & Gr 294 & Hal 513 & M 229 & Nāmī B 28 & RÉS 3006 & $\begin{array}{l}\text { Bauer \& Lundin 1998: } 109 \text {; } \\
\text { Garbini } 1974\end{array}$ \\
\hline B-M 227 & Gr 296 & & M 466 & & & $\begin{array}{l}\text { Bauer \& Lundin 1998: 109- } \\
110 \text {; Garbini } 1974\end{array}$ \\
\hline B-M 228 & Gr 295 & Hal 464 & M 184 & Nāmī B 29 & RÉS 2964 & $\begin{array}{l}\text { Bauer \& Lundin 1998: 109; } \\
\text { Garbini } 1974\end{array}$ \\
\hline B-M 229 & & & M 183 & & RÉS 2963 & Garbini 1974 \\
\hline \multicolumn{7}{|l|}{ B-M 230} \\
\hline $\begin{array}{l}\text { B-M } 231= \\
\text { Robin- } \\
\text { Barāqish } 50 \\
\text { [frag. de Bauer } \\
5=\text { B-M } 231+ \\
224+238 \text { ] }\end{array}$ & Gr 299 & & & & & Bauer \& Lundin $1998: 110$ \\
\hline B-M 232 & Gr 298 & Hal 515 & M 231 & & RÉS 3008 & $\begin{array}{l}\text { Bauer \& Lundin 1998: } 110 \text {; } \\
\text { Garbini } 1974\end{array}$ \\
\hline B-M 233 & Gr 297 & Hal 514 & M 230 & & RÉS 3007 & $\begin{array}{l}\text { Bauer \& Lundin 1998: } 110 \text {; } \\
\text { Garbini } 1974\end{array}$ \\
\hline B-M 234 & & & M 161 & & RÉS 2939 & Garbini 1974 \\
\hline \multicolumn{7}{|l|}{ B-M 235} \\
\hline B-M 236 & Gr 301 & & & & & Bauer \& Lundin $1998: 110$ \\
\hline B-M 237 & & & M 404 & Nāmī B. 32 & & Garbini 1974 \\
\hline $\begin{array}{l}\text { B-M } 238 \\
\text { [frag. de Bauer } \\
5=\text { B-M } 231+ \\
224+238]\end{array}$ & Gr 303 & & M 347 & Nāmī B 33 & $R E ́ S 3535$ & $\begin{array}{l}\text { Bauer \& Lundin 1998: 110- } \\
112 ; \text { Garbini 1974; Robin } \\
\text { 1979a: } 104\end{array}$ \\
\hline B-M 239 & & & M 419 & Nāmī B. 87 & & Garbini 1974 \\
\hline B-M 240 & & & M 418 & Nāmī B. 86 & & Garbini 1974 \\
\hline B-M 241 & Gr 304 & $\begin{array}{l}\text { Hal } 465 \\
+466\end{array}$ & M 185 & Nāmī B 34 & $R E ́ S 2965$ & $\begin{array}{l}\text { Bauer \& Lundin 1998: } 113 \text {; } \\
\text { Robin 1979a : 104; 1979b : } \\
\text { 193; Garbini 1974 }\end{array}$ \\
\hline \multicolumn{7}{|l|}{ B-M 242} \\
\hline B-M 243 & & & M 232 & & RÉS 3009 & Garbini 1974 \\
\hline \multicolumn{7}{|l|}{ B-M 244} \\
\hline \multicolumn{7}{|l|}{ B-M 245} \\
\hline B-M 246 & & & M 187 & & RÉS 2967 & Garbini 1974 \\
\hline
\end{tabular}




\begin{tabular}{|c|c|c|c|c|c|c|}
\hline B-M $247+255$ & & & $\begin{array}{l}\text { M } 186+ \\
188\end{array}$ & & $\begin{array}{l}\text { RÉS } 2966 \\
+2968\end{array}$ & $\begin{array}{l}\text { Bauer \& Lundin 1998: } 114 \text {; } \\
\text { Garbini } 1974\end{array}$ \\
\hline B-M 248 & & & M 233 & & RÉS 3010 & Garbini 1974 \\
\hline \multicolumn{7}{|l|}{ B-M 249} \\
\hline \multicolumn{7}{|l|}{ B-M 250} \\
\hline \multicolumn{7}{|l|}{ B-M 251} \\
\hline \multicolumn{7}{|l|}{ B-M 252} \\
\hline B-M 253 & $\begin{array}{l}\text { Gr } 307 \\
+306\end{array}$ & & $\begin{array}{l}\text { M } 405+ \\
189\end{array}$ & $\begin{array}{l}\text { Nāmī B. } 40 \\
+39\end{array}$ & RÉS 2969 & $\begin{array}{l}\text { Bauer \& Lundin 1998 : 114- } \\
115 \text {; Garbini } 1974\end{array}$ \\
\hline \multicolumn{7}{|l|}{ B-M 254} \\
\hline B-M 256 & $\begin{array}{lr}\text { Gr } & 309 \\
+ & \mathrm{Gr} \\
310 & + \\
\mathrm{Gr} & 312 \\
+ & \mathrm{Gr} \\
311 & \end{array}$ & $\begin{array}{l}\text { Hal } 471 \\
+472+ \\
474 \quad+ \\
473\end{array}$ & $\begin{array}{ll}\text { M 194 + } \\
190+ \\
191+ \\
193+ \\
192\end{array}$ & & $\begin{array}{l}\text { RÉS } 2972 \\
+2970+ \\
2971+ \\
2971 \text { bis } \\
\mathrm{B}+2971 \\
\text { bis A }\end{array}$ & $\begin{array}{l}\text { Bauer \& Lundin 1998: 115, } \\
\text { 116; Garbini 1974; Robin } \\
\text { 1979a: } 104\end{array}$ \\
\hline B-M 257 & Gr 313 & $\begin{array}{l}\text { Hal } 535 \\
+578\end{array}$ & M 247 & Nāmī B 46 & RÉS 3022 & $\begin{array}{l}\text { Bauer \& Lundin 1998: 116- } \\
117 ; \text { Garbini 1974; Robin } \\
1979 \mathrm{a}: 104\end{array}$ \\
\hline B-M 258 & Gr 314 & Hal 477 & M 196 & Nāmī B 47 & RÉS 2974 & $\begin{array}{l}\text { Bauer \& Lundin 1998: } 117 \text {; } \\
\text { Garbini } 1974\end{array}$ \\
\hline B-M 259 & Gr 315 & Hal 476 & M 195 & Nāmī B 45 & $R E ́ S 2973$ & $\begin{array}{l}\text { Bauer \& Lundin 1998 : 117- } \\
\text { 118; Garbini } 1974\end{array}$ \\
\hline \multicolumn{7}{|l|}{ B-M 260} \\
\hline B-M 261 & & & M 406 & Nāmī B. 44 & & Garbini 1974 \\
\hline B-M 262 & & & M 210 & & RÉS 2987 & Garbini 1974 \\
\hline \multicolumn{7}{|l|}{ B-M 263} \\
\hline B-M 264 & & & M 239 & & RÉS 3015 & Garbini 1974 \\
\hline B-M 265 & & & M 240 & & RÉS 3016 & Garbini 1974 \\
\hline B-M 266 & & & M 378 & & $R E ́ S 4224$ & Garbini 1974 \\
\hline B-M 267 & & & M 242 & & $\begin{array}{l}R E ́ S 3017 \\
\text { bis }\end{array}$ & $\begin{array}{l}\text { Garbini 1974; Müller } 1982 \text { : } \\
133\end{array}$ \\
\hline B-M 268 & & & M 241 & & RÉS 3017 & $\begin{array}{l}\text { Garbini 1974; Müller } 1982 \text { : } \\
133\end{array}$ \\
\hline B-M 269 & & & M 243 & & RÉS 3018 & Garbini 1974 \\
\hline \multicolumn{7}{|l|}{ B-M 270} \\
\hline \multicolumn{7}{|l|}{ B-M 271} \\
\hline B-M 272 & & & M 246 & & $R E ́ S 3021$ & $\begin{array}{l}\text { Garbini 1974; Müller } 1982: \\
\text { 134; Robin 1979a : } 103\end{array}$ \\
\hline B-M 273 & & & M 200 & & RÉS 2978 & $\begin{array}{l}\text { Garbini 1974; Robin 1979b, } \\
103\end{array}$ \\
\hline \multicolumn{7}{|l|}{ B-M 274} \\
\hline \multicolumn{7}{|l|}{ B-M 275} \\
\hline B-M 276 & & & M 245 & & RÉS 3020 & Garbini 1974 \\
\hline B-M 277 & & & M 244 & & RÉS 3019 & Garbini 1974 \\
\hline B-M 278 & & & M 199 & & RÉS 2977 & Garbini 1974 \\
\hline B-M 279 & & & M 201 & & RÉS 2979 & Garbini 1974 \\
\hline \multirow[t]{8}{*}{ B-M 280} & Gr 317 & Hal 480 & M 198 & Nāmī B 61 & $R E ́ S 2976$ & $\begin{array}{l}\text { Bauer \& Lundin 1998: } 120 \text {; } \\
\text { Garbini } 1974\end{array}$ \\
\hline & Gr 251 & & & Nāmī B 117 & & Bauer \& Lundin $1998: 118$ \\
\hline & Gr 281 & & M 445 & Nāmī B 144 & & $\begin{array}{l}\text { Bauer \& Lundin 1998: } 102 \text {; } \\
\text { Garbini } 1974\end{array}$ \\
\hline & Gr 308 & & & & & Bauer \& Lundin $1998: 115$ \\
\hline & Gr 323 & & M 448 & Nāmī B 155 & & $\begin{array}{l}\text { Bauer \& Lundin 1998: } 121 \text {; } \\
\text { Garbini } 1974\end{array}$ \\
\hline & Gr 286 & & & & & Bauer \& Lundin 1998 : 107 \\
\hline & Gr 280 & & & & & Bauer \& Lundin 1998 : 102 \\
\hline & Gr 326 & & & & & $\begin{array}{l}\text { Bauer \& Lundin 1998: 122- } \\
123\end{array}$ \\
\hline
\end{tabular}




\begin{tabular}{|c|c|c|c|c|c|c|}
\hline & Gr 325 & & & & & Bauer \& Lundin $1998: 122$ \\
\hline & Gr 300 & & & & & Bauer \& Lundin $1998: 110$ \\
\hline & Gr 265 & Hal 503 & M 221 & & RÉS 2998 & $\begin{array}{l}\text { Bauer \& Lundin 1998: } 92 \text {; } \\
\text { Garbini 1974 }\end{array}$ \\
\hline & Gr 324 & Hal 575 & $\begin{array}{l}\text { M } 281= \\
449\end{array}$ & Nāmī B 156 & RÉS 3058 & $\begin{array}{l}\text { Bauer \& Lundin 1998: } 121 \text {; } \\
\text { Garbini } 1974\end{array}$ \\
\hline & & & M 439 & Nami B 116 & & Garbini 1974 \\
\hline $\begin{array}{l}\text { Y.03.B.R44- } \\
45.1\end{array}$ & & & & & & Agostini (dans ce volume) \\
\hline $\begin{array}{l}\text { Y.03.B.R44- } \\
45.2 \text { bis }+2 \text { ter }+ \\
2\end{array}$ & & & & & & Agostini (dans ce volume) \\
\hline $\begin{array}{l}\text { Y.03.B.R44- } \\
45.3\end{array}$ & & & & & & Agostini (dans ce volume) \\
\hline Y.04.B.T45.1 & & & & & & Agostini (dans ce volume) \\
\hline Y.04.B.T45.2 & & & & & & Agostini (dans ce volume) \\
\hline Y.04.B.T45.3 & & & & & & Agostini (dans ce volume) \\
\hline \multirow[t]{4}{*}{ Y.04.B.T45.4 } & & & & & & Agostini (dans ce volume) \\
\hline & & & M 159 & & $\begin{array}{l}R E S S \\
2937\end{array}$ & Garbini 1974 \\
\hline & & & M 262 & & $\begin{array}{l}\text { RÉS } \\
3036\end{array}$ & Garbini 1974 \\
\hline & & & M 263 & & $\begin{array}{l}\text { RES } \\
3037\end{array}$ & Garbini 1974 \\
\hline
\end{tabular}

\subsubsection{Extra muros - Area C}

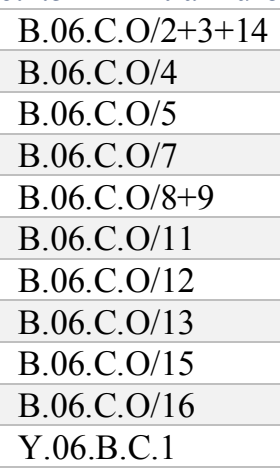

6.1.6 Extra muros - Area D, nécropole

$$
\text { B.05.D.O./10 }
$$

\section{B.05.D.O./11}

B.05.D.O./12

B.05.D.O./13

B.05.D.O./15

B.05.D.O./16

B.05.D.O./18

B.05.D.O. $/ 20$

B.05.D.O. $/ 21$

B.05.D.O./22

B.05.D.O./23
Agostini (dans ce volume) Agostini (dans ce volume) Agostini (dans ce volume) Agostini (dans ce volume) Agostini (dans ce volume) Agostini (dans ce volume) Agostini (dans ce volume) Agostini (dans ce volume) Agostini (dans ce volume) Agostini (dans ce volume) Agostini (dans ce volume)

Antonini \& Agostini $2010: 18$, 52

Antonini \& Agostini 2010 : 21, 52-53

Antonini \& Agostini 2010 : 19, 53

Antonini \& Agostini 2010 : 53

Antonini \& Agostini 2010 : 16-17, 53

Antonini \& Agostini 2010 : 18, 54

Antonini \& Agostini 2010 : 17, 54-55

Antonini \& Agostini 2010 : 17, 55

Antonini \& Agostini $2010: 55$

Antonini \& Agostini 2010 : 18-19, 55

Antonini \& Agostini 2010 : 17, 56 


\begin{tabular}{|c|c|}
\hline B.05.D.O./24 & $\begin{array}{l}\text { Antonini \& Agostini } 2010: 20 \text {, } \\
56\end{array}$ \\
\hline B.05.D.O./25 & $\begin{array}{l}\text { Antonini \& Agostini } 2010: 20 \text {, } \\
56-57\end{array}$ \\
\hline B.05.D.O./26 & $\begin{array}{l}\text { Antonini \& Agostini } 2010: 20 \text {, } \\
57\end{array}$ \\
\hline B.05.D.O./3 & Antonini \& Agostini $2010: 51$ \\
\hline B.05.D.O./33 & $\begin{array}{l}\text { Antonini \& Agostini } 2010: 19 \text {, } \\
58\end{array}$ \\
\hline B.05.D.O./4 & Antonini \& Agostini $2010: 51$ \\
\hline B.05.D.O./9 & $\begin{array}{l}\text { Antonini \& Agostini } 2010: 21 \text {, } \\
51\end{array}$ \\
\hline B.06.D.O./13 & $\begin{array}{l}\text { Antonini \& Agostini } 2010: 17 \text {, } \\
58\end{array}$ \\
\hline B.06.D.O./15 & $\begin{array}{l}\text { Antonini \& Agostini } 2010: 22 \text {, } \\
58-59\end{array}$ \\
\hline B.06.D.O./16 & $\begin{array}{l}\text { Antonini \& Agostini } 2010: 17 \text {, } \\
59\end{array}$ \\
\hline B.06.D.O./17 & $\begin{array}{l}\text { Antonini \& Agostini } 2010: 17 \text {, } \\
59\end{array}$ \\
\hline B.06.D.O./18 & $\begin{array}{l}\text { Antonini \& Agostini } 2010: 22 \text {, } \\
59\end{array}$ \\
\hline B.06.D.O./19 & Antonini \& Agostini $2010: 60$ \\
\hline B.06.D.O./20 & $\begin{array}{l}\text { Antonini \& Agostini } 2010: 18 \text {, } \\
60\end{array}$ \\
\hline B.06.D.O./21 & $\begin{array}{l}\text { Antonini \& Agostini } 2010: 19 \text {, } \\
60-61\end{array}$ \\
\hline B.06.D.O./23 & $\begin{array}{l}\text { Antonini \& Agostini } 2010: 22 \text {, } \\
61\end{array}$ \\
\hline B.06.D.O./24 & $\begin{array}{l}\text { Antonini \& Agostini } 2010: 21 \text {, } \\
61\end{array}$ \\
\hline B.06.D.O./25 & $\begin{array}{l}\text { Antonini \& Agostini } 2010: 24 \text {, } \\
65\end{array}$ \\
\hline B.06.D.O./26 & $\begin{array}{l}\text { Antonini \& Agostini } 2010: 18 \text {, } \\
62\end{array}$ \\
\hline B.06.D.O./27 & $\begin{array}{l}\text { Antonini \& Agostini } 2010: 18 \text {, } \\
62\end{array}$ \\
\hline B.06.D.O./30 & $\begin{array}{l}\text { Antonini \& Agostini } 2010: 18 \text {, } \\
62-63\end{array}$ \\
\hline B.06.D.O./31 & $\begin{array}{l}\text { Antonini \& Agostini } 2010: 19 \text {, } \\
63\end{array}$ \\
\hline MAIRY.05/11 & $\begin{array}{l}\text { Antonini \& Agostini } 2010: 65 \text {, } \\
81\end{array}$ \\
\hline MAIRY.05/12 & Antonini \& Agostini $2010: 66$ \\
\hline MAIRY.05/14 & $\begin{array}{l}\text { Antonini \& Agostini } 2010: 66 \text {, } \\
81\end{array}$ \\
\hline MAIRY.05/16 & $\begin{array}{l}\text { Antonini \& Agostini } 2010: 64 \text {, } \\
77\end{array}$ \\
\hline MAIRY.05/17 & $\begin{array}{l}\text { Antonini \& Agostini } 2010: 64 \text {, } \\
77\end{array}$ \\
\hline MAIRY.05/18 & Antonini \& Agostini $2010: 64$ \\
\hline MAIRY.05/6 & $\begin{array}{l}\text { Antonini \& Agostini 2010: } \\
63-64,78\end{array}$ \\
\hline MAIRY.05/7 & $\begin{array}{l}\text { Antonini \& Agostini } 2010: 64 \text {, } \\
76\end{array}$ \\
\hline MAIRY.05/9 & $\begin{array}{l}\text { Antonini \& Agostini } 2010: 64 \text {, } \\
77\end{array}$ \\
\hline MAIRY.06/3 & $\begin{array}{l}\text { Antonini \& Agostini } 2010: 65 \text {, } \\
79\end{array}$ \\
\hline
\end{tabular}


6.1.7 Extra muros - Indéterminé

Y.86.BAR/13

Antonini $1988: 133$

6.1.8 Indéterminé

\begin{tabular}{|c|c|c|c|c|}
\hline B 31 & Gr 254 & & & Bauer \& Lundin $1998: 90$ \\
\hline BM 125142 & & M 305 & $R E ́ S 3326$ & Garbini 1974 \\
\hline Fa 126 & & M 209 & RÉS 2986 & Garbini 1974 \\
\hline Nami-B 147 & & M 447 & & Garbini 1974 \\
\hline Nami-B 158 & & M 450 & & Garbini 1974 \\
\hline YM 28336 & & & & Arbach \& Audouin $2007: 64$ \\
\hline \multirow[t]{9}{*}{ YM 28988} & & & & Arbach \& Audouin $2007: 67$ \\
\hline & & M 162 & $R E ́ S 2940$ & Garbini 1974 \\
\hline & & M 175 & $R E ́ S 2955$ & Garbini 1974 \\
\hline & & M 176 & $R E ́ S 2956$ & Garbini 1974 \\
\hline & & M 205 & $R E ́ S 2982$ & Garbini 1974 \\
\hline & & M 234 & $R E ́ S 3011$ & Garbini 1974 \\
\hline & & M 235 & $R E ́ S 3011$ bis & Garbini 1974 \\
\hline & & M 270 & RÉS 3044 & Garbini 1974 \\
\hline & & M 337 & RÉS 3403 & Garbini 1974 \\
\hline
\end{tabular}

\subsection{Ad-Darb (Darb al-Ashrāf)}

Y.90.DA 1

Y.90.DA 2

\subsection{Darb aṣ-Ṣabī - Temple de Nakraḥ}

MAFRAY-Darb aṣ-Șabī 1

MAFRAY-Darb aș-Șabī 10

MAFRAY-Darb aṣ-Șabī 11

MAFRAY-Darb aṣ-Șabī 12

MAFRAY-Darb aṣ-Șabī 13

MAFRAY-Darb aṣ-Șabī 15

MAFRAY-Darb aș-Șabī 16

MAFRAY-Darb aș-Șabī 17

MAFRAY-Darb aṣ-Șabī 18

MAFRAY-Darb aṣ-Șabī 19

MAFRAY-Darb aș-Șabī 2

MAFRAY-Darb aș-Șabī 20

MAFRAY-Darb aș-Ṣabī 21

MAFRAY-Darb aș-Șabī 22

MAFRAY-Darb aṣ-Șabī 23

MAFRAY-Darb aṣ-Șabī 24

MAFRAY-Darb aș-Ṣabī 25

MAFRAY-Darb aṣ-Șabī 26

MAFRAY-Darb aș-Șabī 27

MAFRAY-Darb aș-Șabī 28

MAFRAY-Darb aṣ-Șabī 29

MAFRAY-Darb aṣ-Șabī 3

MAFRAY-Darb aṣ-Șabī 30

MAFRAY-Darb aș-Ṣabī 31

MAFRAY-Darb aș-Șabī 32

MAFRAY-Darb aṣ-Șabī 4

MAFRAY-Darb aș-Ṣabī 5

MAFRAY-Darb aṣ-Ṣabī 6

Robin et al. $1988: 99-109$

Robin et al. 1988 : 120-121

Robin et al. $1988: 121-122$

Robin et al. $1988: 122$

Robin et al. 1988 : 123-124

Robin et al. $1988: 124-125$

Robin et al. 1988 : 126-128

Robin et al. $1988: 128-129$

Robin et al. 1988 : 129-130

Robin et al. $1988: 130$

Robin et al. 1988 : 109-111

Robin et al. $1988: 131$

Robin et al. $1988: 131-132$

Robin et al. $1988: 132$

Robin et al. $1988: 133$

Robin et al. $1988: 133$

Robin et al. 1988 : 133-134

Robin et al. $1988: 134-136$

Robin et al. 1988 : 136-137

Robin et al. 1988 : 111-112

Robin et al. $1988: 137-138$

Robin et al. $1988: 138$

Arbach 1994 : 5-8

Robin et al. $1988: 113$

Robin et al. 1988 : 114-115

Robin et al. $1988: 116$ 


\section{4 Ḥuṣn Āl Ṣālị̣}

MAFRAY-Ḥuṣn Āl Șālị̣ 1

Robin $1987: 168$

MAFRAY-Ḥuṣn Āl Șāliḥ 2

Robin 1987 : 169-171

MAFRAY-Huș Āl Șālih 3

MAFRAY-Hușn Āl Șālih 4

MAFRAY-Ḥuṣn Āl Șāliḥ 5

6.5 Shaqab al-Manașșa - Temple de 'Athtar dhu-Yahriq

\begin{tabular}{|c|c|c|c|c|c|c|}
\hline Shaqab 1 & & & $\begin{array}{l}\text { MAFRAY-ash- } \\
\text { Shaqab } 3\end{array}$ & & & Gnoli 1993 : 69-72 \\
\hline Shaqab 2 & & & $\begin{array}{l}\text { MAFRAY-ash- } \\
\text { Shaqab } 4\end{array}$ & & & Gnoli 1993 : 72-74 \\
\hline Shaqab 3 & & & $\begin{array}{l}\text { MAFRAY-ash- } \\
\text { Shaqab } 5\end{array}$ & & & Gnoli 1993 : 74-75 \\
\hline Shaqab 4 & & & $\begin{array}{l}\text { MAFRAY-ash- } \\
\text { Shaqab } 1\end{array}$ & & & Gnoli 1993 : 75-78 \\
\hline Shaqab 5 & & & $\begin{array}{l}\text { MAFRAY-ash- } \\
\text { Shaqab } 2\end{array}$ & & & Gnoli 1993 : 78-80 \\
\hline Shaqab 6 & & & & & $\begin{array}{l}\text { Y.90.SHQ } \\
2\end{array}$ & Gnoli 1993 : 80-82 \\
\hline Shaqab 7 & & & $\begin{array}{l}\text { MAFRAY-ash- } \\
\text { Shaqab } 7\end{array}$ & & & Gnoli 1993 : 83-84 \\
\hline Shaqab 8 & & & & & $\begin{array}{l}\text { Y.86.SHQ } \\
1\end{array}$ & Gnoli 1993 : 84-86 \\
\hline Shaqab 9 & & & & & $\begin{array}{l}\text { Y.92.SHQ } \\
3\end{array}$ & Gnoli 1993 : 86-87 \\
\hline Shaqab 10 & & & $\begin{array}{l}\text { MAFRAY-ash- } \\
\text { Shaqab } 8\end{array}$ & & & Gnoli $1993: 87$ \\
\hline Shaqab 11 & & & $\begin{array}{l}\text { MAFRAY-ash- } \\
\text { Shaqab } 11\end{array}$ & & & Gnoli $1993: 87$ \\
\hline Shaqab 12 & & & $\begin{array}{l}\text { MAFRAY-ash- } \\
\text { Shaqab } 6\end{array}$ & & & Gnoli $1993: 88$ \\
\hline Shaqab 13 & & & $\begin{array}{l}\text { MAFRAY-ash- } \\
\text { Shaqab } 9\end{array}$ & & & Gnoli 1993 : 88-89 \\
\hline Shaqab 14 & & & $\begin{array}{l}\text { MAFRAY-ash- } \\
\text { Shaqab } 10\end{array}$ & & & Gnoli $1993: 89$ \\
\hline Shaqab 15 & & & & & $\begin{array}{l}\text { Y.92.SHQ } \\
4\end{array}$ & Gnoli 1993 : 91-92 \\
\hline Shaqab 16 & & & $\begin{array}{l}\text { MAFRAY-ash- } \\
\text { Shaqab } 12\end{array}$ & & & Gnoli 1993 : 92-95 \\
\hline Shaqab 17 & & & $\begin{array}{l}\text { MAFRAY-ash- } \\
\text { Shaqab } 14\end{array}$ & & & Gnoli 1993 : 95 \\
\hline Shaqab 18 & & & $\begin{array}{l}\text { MAFRAY-ash- } \\
\text { Shaqab } 13\end{array}$ & & & $\begin{array}{l}\text { Gnoli } 1993: 95-7 \text {; Robin } \\
1988: 102-3\end{array}$ \\
\hline Shaqab 19 & Fa 14 & $\begin{array}{l}\text { Hal } \\
484\end{array}$ & M 202 & $\begin{array}{l}R E S \\
2980\end{array}$ & & $\begin{array}{l}\text { Garbini } 1974 \text {; Gnoli } 1993 \text { : } \\
100-112\end{array}$ \\
\hline
\end{tabular}

\subsection{Wādī Malāhāā}

MAFRAY-Malāḥā' 2

MAFRAY-Malāḥā 1

Robin 1987 : 171-172

MAFRAY-Malāhā' 3

Robin 1988 : 101-102 
7 Annexe 2 - Liste des constructions attestées dans les inscriptions de Barāqish

\begin{tabular}{|c|c|c|c|c|c|c|}
\hline \multirow{2}{*}{$\begin{array}{l}\text { Nom de } \\
\text { la } \\
\text { structur } \\
\text { e }\end{array}$} & \multirow[b]{2}{*}{ Nature } & \multicolumn{2}{|c|}{ Commanditaires } & \multirow[b]{2}{*}{ Règne } & \multirow[b]{2}{*}{ Date } & \multirow[b]{2}{*}{ Inscription } \\
\hline & & $\begin{array}{l}\text { Fraction } \\
\text { de clan }\end{array}$ & Clan & & & \\
\hline- & $\begin{array}{l}30 \text { coudées de } \\
\text { muraille }\end{array}$ & $Y d^{\circ}$ & $?$ & $?$ & $6^{\mathrm{e}}-5^{\mathrm{e}}$ av. & Ma īn 6 \\
\hline$?$ & Bassins (qldn) & $\begin{array}{l}\dot{G} r b t \\
H \underline{d} d k t\end{array}$ & $\begin{array}{l}\text { Mwqh / } \\
\text { Qrn }\end{array}$ & $?$ & $5^{\mathrm{e}}-1^{\mathrm{e}}$ av. & $\begin{array}{l}\text { MAFRAY-Darb } \\
\text { aș-Șabī } 3\end{array}$ \\
\hline$?$ & $\begin{array}{l}\text { Courtine } \\
(s, h f t n)\end{array}$ & $?$ & $?$ & 'lyf' Wqh w-Wqh'l $S d q$ & $5^{\mathrm{e}}$ av. & B-M $247+255$ \\
\hline$?$ & Maison (byt) & Blh & $G b^{\prime} n$ & ${ }^{\prime} b y d^{c} Y \underline{t}^{\mathrm{c} 34}$ & $5^{\mathrm{e}}$ av. & Y.03.B.R44-45.3 \\
\hline$?$ & $\begin{array}{l}\text { Bastion } \\
(m h f d n)\end{array}$ & $Y f^{\prime \prime} n ?^{35}$ & $G b^{\prime} n ?$ & $\begin{array}{l}{ }^{\prime b y d} d^{`} \quad Y \underline{t}^{c} w-b n-s^{1} W q h^{\prime} l \\
\text { Rym }\end{array}$ & $5^{\mathrm{e}}$ av. & $\begin{array}{l}\text { B-M } 180=R E ́ S \\
2942\end{array}$ \\
\hline$?$ & $\begin{array}{l}\text { Courtine } \\
(\operatorname{shftn})\end{array}$ & $\dot{G} z r-S^{\prime} h f n$ & $?$ & Wqh'l Rym & $5^{\mathrm{e}}-4^{\mathrm{e}}$ av & $\begin{array}{l}\mathrm{B}-\mathrm{M} 222=R E ́ S \\
3005\end{array}$ \\
\hline$?$ & $\begin{array}{l}\text { Courtine } \\
(\operatorname{shftn})\end{array}$ & Royal & Royal & 'bkrb $S d q$ & $4^{e}$ av. & B-M 249 \\
\hline$?$ & $\begin{array}{l}\text { Bastion } \\
(m h f d n)\end{array}$ & Royal & Royal & 'bkrb $S d q$ & $4^{e}$ av. & B-M 103 \\
\hline$?$ & Portique $(r s f)$ & $\begin{array}{l}\text { Hbrr } \\
S^{2} y d\end{array}$ & $?$ & 'bkrb S Sdq bn Wqh'l & $4^{\mathrm{e}}$ av. & Shaqab 4 \\
\hline$?$ & Portique $(r s f)$ & $\begin{array}{l}S^{2} g^{\prime} \\
S^{2} y d\end{array}$ & $?$ & $Y \underline{t}^{\prime} \mathrm{l} / \mathrm{Rym}$ & $4^{\mathrm{e}}$ av. & Shaqab 5 \\
\hline$?$ & Puits $\left(b^{\prime} r\right)$ & ${ }^{c} s^{1} d n$ & $?$ & $?$ & $4^{\mathrm{e}}-2^{\mathrm{e}}$ av. & Shaqab 16 \\
\hline$?$ & $\begin{array}{l}\text { Bastion } \\
(m h f d n)\end{array}$ & $M r n$ & $?$ & $?$ & $2^{\mathrm{e}}-1^{\mathrm{e}}$ av & $\begin{array}{l}\text { B-M } 218=R E ́ S \\
2960\end{array}$ \\
\hline$[\ldots] \mathbf{R}^{\prime} \mathbf{N}$ & $\begin{array}{l}\text { Bastion } \\
(m h f d n)\end{array}$ & $S^{2 c} \underline{t} m$ & $G b^{\prime} n$ & $\begin{array}{l}\text { 'lyf } Y s^{2} r \quad w-b n-s^{1} H f n m \\
\text { Rym }\end{array}$ & $3^{\mathrm{e}}-2^{\mathrm{e}}$ av. & $\begin{array}{l}\text { B-M } 272=R E ́ S \\
3021\end{array}$ \\
\hline 'BRM & $\begin{array}{l}\text { Courtine } \\
([s, h] f t n)(?)\end{array}$ & $?$ & $?$ & $W q h^{\prime} l[N b t ?]$ & $3^{e}-2^{e}$ av. & B-M 108 \\
\hline \multirow{4}{*}{$B R N$} & $\begin{array}{l}\text { Temple (byt) } \\
\text { Aménagement } \\
\text { intérieur }\end{array}$ & Blh & $G b^{\prime} n$ & 'byd ${ }^{c} Y \underline{t}^{c}$ & $5^{\mathrm{e}}$ av. & Y.92.B.A $21+30$ \\
\hline & - & $s^{1} d n$ & $?$ & 'byd' $Y \underline{t^{c}}$ & $5^{\mathrm{e}}$ av. & $\begin{array}{l}\text { B-M } 216=R E ́ S \\
2959\end{array}$ \\
\hline & $\begin{array}{l}\text { [Brn n'est pas } \\
\text { nommé] } \\
\text { Table } \\
\text { offrande } \\
(m s ̦ r b) \\
\text { Banquettes } \\
(t w t b) \\
\text { Piliers }(h w r)\end{array}$ & $S^{2} \underline{t} m$ & $G b^{\prime} n$ & 'byd' $Y \underline{t}^{c}(?)$ & $5^{\mathrm{e}}$ av. & Y.90.B.A.7 \\
\hline & $\begin{array}{l}\text { [Brn n'est pas } \\
\text { nommé] } \\
\text { Adyton }(g w b) \\
\text { Naos }(m k n t)\end{array}$ & $D b r$ & $Y l q z$ & $Y \underline{Y}^{\prime \prime l} \operatorname{Rym} w-b n-s^{1} T b^{`} k r b$ & $4^{e}$ av. & RÉS 2980 bis \\
\hline $\begin{array}{l}B Y T \\
W D M\end{array}$ & Temple (byt) & $S^{2} \underline{\underline{t}} m$ & $G b^{\prime} n$ & 'byd' $Y \underline{t}^{c}(?)$ & $5^{\mathrm{e}}$ av. & $\begin{array}{l}\text { B-M } 277=R E ́ S \\
3019\end{array}$ \\
\hline $\begin{array}{l}\text { D- } \\
B H R N\end{array}$ & $\begin{array}{l}\text { Répartiteur (?) } \\
(m z f y)\end{array}$ & $\begin{array}{l}\text { Hbrr } \\
S^{l} y l\end{array}$ & $? /$ 'ly'l & ${ }^{2} l y f^{c} Y s^{2} r w-b n-s^{1} H w f^{t} t t$ & $3^{\mathrm{e}}-2^{\mathrm{e}}$ av. & $\begin{array}{l}\text { MAFRAY- } \\
\text { Malāḥā } 1\end{array}$ \\
\hline
\end{tabular}

\footnotetext{
${ }^{34}$ En considérant que l'auteur est le même ' $m s^{I} m$ ' kabīr de Yathill que dans les inscriptions RÉS 2959, RÉS 3022 et Y.92.B.A $21+30$ toutes trois datées du règne de 'byd' $Y \underline{t}$ '.

${ }^{35}$ Nous faisons l'hypothèse que les auteurs de l'inscription apparaissent aussi dans l'inscription RÉS 3012.
} 


\begin{tabular}{|c|c|c|c|c|c|c|}
\hline$D-B Q R N$ & $\begin{array}{l}\text { Bastion } \\
(m h f d n)\end{array}$ & $H f d$ & $G b^{\prime} n$ & 'bkrb $S d q$ & $4^{e}$ av. & $\begin{array}{l}\text { B-M } 241=R E ́ S \\
2965\end{array}$ \\
\hline D-HFN & $\begin{array}{l}\text { Bastion } \\
(m h f d n)\end{array}$ & $H f n$ & $G b^{\prime} n$ & 'byd' Y $\underline{t^{\prime}} w$-Wqh'l Rym & $5^{\mathrm{e}}$ av. & $\begin{array}{l}\text { B-M } 182=R E S \\
2929+2941+ \\
2945+2946\end{array}$ \\
\hline $\begin{array}{l}\text { D- } \\
H S B R\end{array}$ & $\begin{array}{l}\text { Courtine } \\
(\text { shftn) }\end{array}$ & $S^{2} \underline{t} m$ & $G b^{\prime} n$ & $\begin{array}{l}\text { 'lyf } Y s^{2} r \quad w-b n-s^{1} \quad H f n m \\
\text { Rym }\end{array}$ & $3^{\mathrm{e}}-2^{\mathrm{e}}$ av. & $\begin{array}{l}\text { B-M } 272=R E ́ S \\
3021\end{array}$ \\
\hline \multirow{2}{*}{$\underline{D}-M L H$} & \multirow{2}{*}{$\begin{array}{l}\text { Bastion } \\
(m h f d n)\end{array}$} & $Y f^{\prime} n$ & $G b^{\prime} n$ & 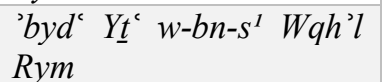 & $5^{\mathrm{e}}$ av. & $\begin{array}{l}\mathrm{B}-\mathrm{M} 10=R \dot{E} S \\
3012\end{array}$ \\
\hline & & Zlwmn & $G b^{\prime} n$ & $' l y f^{\mathrm{e}} Y s^{2} r w-\left(W q h^{\prime} l ?\right) N b t$ & $3^{\mathrm{e}}-2^{\mathrm{e}}$ av. & $\begin{array}{l}\mathrm{B}-\mathrm{M} 1=R E ́ S \\
2975\end{array}$ \\
\hline \multirow{3}{*}{$\begin{array}{l}\text { D- } \\
N D B N\end{array}$} & \multirow{3}{*}{$\begin{array}{l}\text { Bastion } \\
(m h f d n)\end{array}$} & $?$ & $?$ & 'byd $d^{c} Y \underline{t}^{c} w-b n-s^{1} H y w m$ & $5^{\mathrm{e}}$ av. & $\begin{array}{l}\text { Y.03.B.R44- } \\
\text { 45.2bis }+2 \text { ter }+2\end{array}$ \\
\hline & & $\dot{G} r b t$ & $M w q h$ & 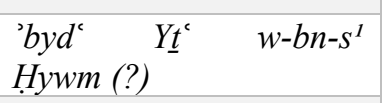 & $5^{\mathrm{e}}$ av. & $\begin{array}{l}\text { Y.92.B.A } 37 \text { A- } \\
\text { B }\end{array}$ \\
\hline & & $H f d$ & $G b^{\prime} n$ & 'bkrb $S d q$ & $4^{\mathrm{e}}$ av. & $\begin{array}{l}\text { B-M } 241=R E ́ S \\
2965\end{array}$ \\
\hline $\begin{array}{l}D- \\
S^{2} F T N\end{array}$ & $\begin{array}{l}\text { Bastion } \\
(m h f d n)\end{array}$ & $H f d$ & $G b^{\prime} n$ & 'bkrb $S d q$ & $4^{\mathrm{e}}$ av. & $\begin{array}{l}\text { B-M } 241=R E ́ S \\
2965\end{array}$ \\
\hline$D D N$ & $\begin{array}{l}\text { Courtine } \\
(s h f t n)\end{array}$ & $Y f^{\prime \prime} n$ & $G b^{\prime} n$ & 'byd' Rym bn Hyw $S d q$ & $4^{\mathrm{e}}$ av. & $\begin{array}{l}\mathrm{B}-\mathrm{M} 56=R E ́ S \\
3060\end{array}$ \\
\hline$H R N$ & $?$ & $Y f^{\prime \prime} n$ & $G b^{\prime} n$ & $\begin{array}{l}{ }^{\prime} b y d^{c} \quad Y \underline{t}^{\mathrm{c}} w-b n-s^{1} W q h^{\prime} l \\
\text { Rym }\end{array}$ & $5^{\mathrm{e}}$ av. & $\begin{array}{l}\mathrm{B}-\mathrm{M} 10=R E ́ S \\
3012\end{array}$ \\
\hline$H R S^{2}$ & $\begin{array}{l}\text { Courtine } \\
(s h f t n)\end{array}$ & $S^{2} \underline{t} m$ & $G b^{\prime} n$ & $\begin{array}{l}\text { 'lyf } Y s^{2} r \quad w-b n-s^{1} H f n m \\
\text { Rym }\end{array}$ & $3^{\mathrm{e}}-2^{\mathrm{e}}$ av. & $\begin{array}{l}\text { B-M } 272=R E ́ S \\
3021\end{array}$ \\
\hline \multirow{4}{*}{$\begin{array}{l}L B^{\prime} N(I) \\
\left(L B^{\prime} N\right. \\
\left.D-{ }^{-} N N\right)\end{array}$} & \multirow{4}{*}{$\begin{array}{l}\text { Bastion } \\
(m h f d n)\end{array}$} & \multirow{2}{*}{ Blh } & \multirow{2}{*}{$G b^{\prime} n$} & \multirow{2}{*}{ 'byd' $Y \underline{t^{c}}$} & \multirow{2}{*}{$5^{\mathrm{e}}$ av. } & B-M 256 \\
\hline & & & & & & Y.92.B.A $21+30$ \\
\hline & & $\begin{array}{l}Y f^{*} n \quad / \\
D f g n\end{array}$ & $\begin{array}{l}G b^{\prime} n / \\
?\end{array}$ & 'byd' $Y \underline{t}^{\mathrm{c}}$ & $5^{\mathrm{e}}$ av. & $\begin{array}{l}\mathrm{B}-\mathrm{M} 257=R \dot{E} S \\
3022\end{array}$ \\
\hline & & $S^{2} \underline{t} t m$ & $G b^{\prime} n$ & $\begin{array}{l}\text { 'lyf } Y s^{2} r w-b n-s^{1} H f n m \\
\text { Rym }\end{array}$ & $3^{\mathrm{e}}-2^{\mathrm{e}}$ av. & $\begin{array}{l}\text { B-M } 272=R E ́ S \\
3021\end{array}$ \\
\hline \multirow{2}{*}{$\begin{array}{l}L B^{\prime} N \\
\text { (II) }\end{array}$} & \multirow{2}{*}{$\begin{array}{l}\text { Bastion } \\
(m h f d n)\end{array}$} & $H f n$ & $G b^{\prime} n$ & 'byd' $Y \underline{t}^{\mathrm{c}} w-W q h^{\prime} l \mathrm{Rym}$ & $5^{\mathrm{e}}$ av. & $\begin{array}{l}\text { B-M } 182=R E ́ S \\
2929+2941+ \\
2945+2946\end{array}$ \\
\hline & & $?$ & $G b^{\prime} n$ & 'byd' $Y t^{\circ} w-W q h^{\prime} l$ Rym & $5^{\mathrm{e}}$ av. & $\begin{array}{l}\text { B-M } 211+\mathrm{B}-\mathrm{M} \\
204=R E S S 2952 \\
+2949\end{array}$ \\
\hline$M D B$ & $\begin{array}{l}\text { Courtine } \\
(s h f t n)\end{array}$ & Dfgn & $?$ & 'byd' $Y \underline{t}^{\mathrm{c}} w-W q h^{\prime} l \mathrm{Rym}$ & $5^{\mathrm{e}}$ av. & $\begin{array}{l}\mathrm{B}-\mathrm{M} 238=R E ́ S \\
3535\end{array}$ \\
\hline MḤpR & Maison (byt) & $?$ & $?$ & $\begin{array}{l}Y \underline{t}^{\prime} l \quad S d q w-b n-s^{1} W q h^{\prime} l \\
Y \underline{t}^{c}\end{array}$ & $\begin{array}{l}1^{\mathrm{e}} \text { av. } \\
\text { v. }-60\end{array}$ & $\begin{array}{l}\text { B-M } 265=R E ́ S \\
3016\end{array}$ \\
\hline \multirow{2}{*}{$N M R N$} & \multirow{2}{*}{$\begin{array}{l}\text { Bastion } \\
(m h f d n)\end{array}$} & $?$ & $?$ & - & $5^{\mathrm{e}}-1^{\mathrm{e}}$ av. & $\begin{array}{l}\text { B-M } 264=R E ́ S \\
3015\end{array}$ \\
\hline & & Royal & Royal & $?$ & $5^{\mathrm{e}}-1^{\mathrm{e}}$ av. & $\begin{array}{l}\mathrm{B}-\mathrm{M} \\
3025\end{array} 6=R E S$ \\
\hline$N^{c} M N$ & Maison (byt) & $?$ & $G b^{\prime} n$ & 'byd' $Y \underline{t}^{\mathrm{c}} w-W q h^{\prime} l \mathrm{Rym}$ & $5^{\mathrm{e}}$ av. & $\begin{array}{l}\text { B-M } 211+\text { B-M } \\
204=R E S S 2952 \\
+2949\end{array}$ \\
\hline$Q B D M$ & Temple (byt) & $\dot{G} z r-S^{\prime} h f n$ & $?$ & Wqh'l Rym & $5^{e}-4^{e}$ av. & Y.05.B.B.13 \\
\hline $\begin{array}{l}\text { RBQN } \\
\text { (I ou II) }\end{array}$ & $\begin{array}{l}\text { Bastion } \\
(m h f d n)\end{array}$ & $?$ & $?$ & 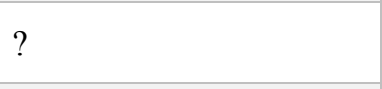 & $5^{\mathrm{e}}-1^{\mathrm{e}}$ av. & Y.92.B.A 16 \\
\hline \multirow{2}{*}{$\begin{array}{l}R B Q N \\
\text { (I) }\end{array}$} & \multirow{2}{*}{$\begin{array}{l}\text { Bastion } \\
(m h f d n)\end{array}$} & $S^{2} \underline{t} m$ & $G b^{\prime} n$ & $\begin{array}{l}\text { 'lyf } Y s^{2} r \quad w-b n-s^{1} \quad H f n m \\
\text { Rym }\end{array}$ & $3^{\mathrm{e}}-2^{\mathrm{e}}$ av. & $\begin{array}{l}\text { B-M } 272=R E S \\
3021\end{array}$ \\
\hline & & Zlwmn & $G b^{\prime} n$ & 'lyf $Y s^{2} r w\left(W q h^{\prime} l ?\right) N b t$ & $3^{\mathrm{e}}-2^{\mathrm{e}}$ av. & $\begin{array}{l}\mathrm{B}-\mathrm{M} 1=R E S \\
2975\end{array}$ \\
\hline $\begin{array}{l}R B Q N \\
\text { (II) }\end{array}$ & $\begin{array}{l}\text { Bastion } \\
(m h f d n)\end{array}$ & $S^{2} \underline{t} m$ & $G b^{\prime} n$ & $\begin{array}{l}\text { 'lyf } Y s^{2} r \quad w-b n-s^{1} H f n m \\
\text { Rym }\end{array}$ & $3^{\mathrm{e}}-2^{\mathrm{e}}$ av. & $\begin{array}{l}\text { B-M } 272=R E ́ S \\
3021\end{array}$ \\
\hline
\end{tabular}




\begin{tabular}{|c|c|c|c|c|c|c|}
\hline$R D W N$ & $\begin{array}{l}\text { Bastion } \\
(m h f d n)\end{array}$ & $S^{2} \underline{t} m$ & $G b^{\prime} n$ & $\begin{array}{l}\text { 'lyf } Y S^{2} r \quad w-b n-s^{1} \quad H f n m \\
\text { Rym }\end{array}$ & $3^{\mathrm{e}}-2^{\mathrm{e}}$ av. & $\begin{array}{l}\text { B-M } 272=R E ́ S \\
3021\end{array}$ \\
\hline$R T^{c}$ & $\begin{array}{l}\text { Courtine } \\
(\text { shftn) }\end{array}$ & $Y f^{\prime \prime} n$ & $G b^{\prime} n$ & 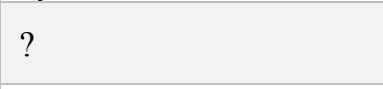 & $5^{\mathrm{e}}-1^{\mathrm{e}}$ av. & $\begin{array}{l}\text { B-M } 266=R E ́ S \\
4224\end{array}$ \\
\hline$S^{\prime} L F$ & $\begin{array}{l}\text { Courtine } \\
(\operatorname{shftn})\end{array}$ & $H f d$ & $G b^{\prime} n$ & 'bkrb $S d q$ & $4^{\mathrm{e}}$ av. & $\begin{array}{l}\text { B-M } 241=R E ́ S \\
2965\end{array}$ \\
\hline$S^{\prime} L F$ & ? (mbny) & $?$ & $?$ & $?$ & $5^{\mathrm{e}}-1^{\mathrm{e}}$ av. & Y.92.B.A 16 \\
\hline$S^{2} B M T$ & 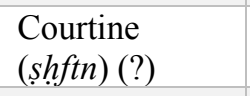 & $Y f^{\prime} n$ & $G b^{\prime} n$ & $\begin{array}{l}\text { 'byd } Y \underline{t}^{`} w-b n-s^{1} W q h^{\prime} l \\
\text { Rym }\end{array}$ & $5^{\mathrm{e}}$ av. & $\begin{array}{l}\mathrm{B}-\mathrm{M} 10=R E ́ S \\
3012\end{array}$ \\
\hline$S^{2} B^{c} N$ & $\operatorname{Bassin}\left(m^{\prime} h \underline{d} \underline{d n}\right)$ & Royal & Royal & Wqh'l Rym & $5^{e}-4^{e}$ av. & Shaqab 18 \\
\hline$S D Q N$ & Maison (byt) & $S^{2} \underline{t} m$ & $G b^{\prime} n$ & Wqh'l Rym & $5^{e}-4^{e}$ av. & $\begin{array}{l}\text { Y.90.B.ext } 2= \\
\text { YM 26117 }\end{array}$ \\
\hline$S D Q N$ & $\begin{array}{l}\text { Bastion } \\
(m h f d n)\end{array}$ & $\dot{G} z r-S^{\prime} h f n$ & $?$ & Wqh'l Rym & $5^{e}-4^{e}$ av. & Y.05.B.B.13 \\
\hline \multirow{2}{*}{$T F S^{2}$} & \multirow{2}{*}{$\begin{array}{l}\text { Courtine } \\
(s h f t n)\end{array}$} & $?$ & $?$ & ${ }^{\prime} b y d^{c} Y \underline{t}^{c} w-b n-s^{l} H y w m$ & $5^{\mathrm{e}}$ av. & $\begin{array}{l}\text { Y.03.B.R44- } \\
\text { 45.2bis + 2ter + } 2\end{array}$ \\
\hline & & $\dot{G} r b t$ & $M w q h$ & $\begin{array}{l}\text { 'byd } Y \underline{t}^{c} w-b n-s^{l} H y w m \\
\text { (?) }\end{array}$ & $5^{\mathrm{e}}$ av. & $\begin{array}{l}\text { Y.92.B.A } 37 \text { A- } \\
\text { B }\end{array}$ \\
\hline$T \dot{G} L$ & Puits $\left(b^{\prime} r\right)$ & $?$ & $G b^{\prime} n$ & 'byd' $Y \underline{t}^{\circ} w-W q h^{\prime} l$ Rym & $5^{\mathrm{e}}$ av. & $\begin{array}{l}\text { B-M } 211+\text { B-M } \\
204=R E ́ S 2952 \\
+2949\end{array}$ \\
\hline \multirow[b]{2}{*}{$T N^{c} M$} & \multirow{2}{*}{$\begin{array}{l}\text { Courtine } \\
(s h f t n)\end{array}$} & $?$ & $?$ & $?$ & $5^{\mathrm{e}}-1^{\mathrm{e}}$ av. & $\begin{array}{l}\text { B-M } 248=R E ́ S \\
3010\end{array}$ \\
\hline & & $\begin{array}{l}Y f^{\prime} n \quad / \\
D f g n\end{array}$ & $\begin{array}{l}G b^{\prime} n / \\
?\end{array}$ & 'byd $Y \underline{t}^{\mathrm{c}}$ & $5^{e}$ av. & $\begin{array}{l}\text { B-M } 257=R E ́ S \\
3022\end{array}$ \\
\hline \multirow{4}{*}{$\begin{array}{l}T S^{2} B M \\
\text { (I) }\end{array}$} & $\begin{array}{l}\text { Courtine } \\
(s h f t n)\end{array}$ & $?$ & $?$ & $?$ & $5^{\mathrm{e}}-1^{\mathrm{e}}$ av. & $\begin{array}{l}\text { B-M } 264=R E ́ S \\
3015: T S^{2}[B M]\end{array}$ \\
\hline & $?$ & $Y f^{\prime} n$ & $G b^{\prime} n$ & $?$ & $5^{e}-1^{e}$ av. & 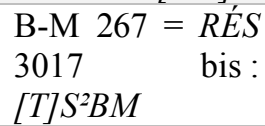 \\
\hline & $\begin{array}{l}\text { Courtine } \\
([s, h] f t n)\end{array}$ & $?$ & $?$ & $?$ & $5^{\mathrm{e}}-1^{\mathrm{e}}$ av. & $\begin{array}{l}\mathrm{B}-\mathrm{M} 7=R E S \\
3024\end{array}$ \\
\hline & $?$ & $Y f^{2} n$ & $G b^{\prime} n$ & $\begin{array}{l}\text { 'byd } Y \underline{t}^{`} w-b n-s^{1} W q h^{\prime} l \\
\text { Rym }\end{array}$ & $5^{\mathrm{e}}$ av. & $\begin{array}{l}\mathrm{B}-\mathrm{M} 10=R E ́ S \\
3012\end{array}$ \\
\hline \multirow{2}{*}{$\begin{array}{l}T S^{2} B M \\
\text { (II) }\end{array}$} & \multirow{2}{*}{$\begin{array}{l}\text { Courtine } \\
(s h f t n)\end{array}$} & \multirow[t]{2}{*}{$?$} & \multirow[t]{2}{*}{$?$} & \multirow[t]{2}{*}{ s. } & \multirow{2}{*}{$5^{\mathrm{e}}-1^{\mathrm{e}}$ av } & $\begin{array}{l}\mathrm{B}-\mathrm{M} 41=R E ́ S \\
3038\end{array}$ \\
\hline & & & & & & B-M 43 \\
\hline$T^{\prime} R M$ & $\begin{array}{l}\text { Courtine } \\
\text { (shftn) }\end{array}$ & Dmrn & $?$ & $\begin{array}{l}W q h^{\prime} l \quad Y \underline{t}^{\circ} \quad w-b n-s^{l} \quad ' l y f^{\circ} \\
Y s^{2} r \\
\text { Synchronisme: } \\
S^{2} h r \text { Ygl Yhrgb mlk Qtbn }\end{array}$ & $\begin{array}{l}1^{\mathrm{e}} \text { av. } \\
\text { v. }-25 /-1\end{array}$ & $\begin{array}{l}\text { B-M } 147=R E ́ S \\
2999\end{array}$ \\
\hline$Y F^{c} N$ & $?$ & $Y f^{2} n$ & $G b^{\prime} n$ & $\begin{array}{l}\text { 'byd } Y \underline{t}^{c} w-b n-s^{1} W q h^{\prime} l \\
\text { Rym }\end{array}$ & $5^{\mathrm{e}}$ av. & $\begin{array}{l}\mathrm{B}-\mathrm{M} 10=R E ́ S \\
3012\end{array}$ \\
\hline$Y \dot{G} L$ & $\begin{array}{l}\text { Bastion } \\
(m h f d n)\end{array}$ & $Y f^{\prime} n$ & $G b^{\prime} n$ & $\begin{array}{l}\text { byd } Y \underline{t}^{`} w-b n-s^{1} W q h^{\prime} l \\
\text { Rym }\end{array}$ & $5^{\mathrm{e}}$ av. & $\begin{array}{l}\mathrm{B}-\mathrm{M} 10=R \dot{E} S \\
3012\end{array}$ \\
\hline YHR & $\begin{array}{l}\text { Bastion } \\
(m h f d n)\end{array}$ & $?$ & $?$ & $?$ & $5^{\mathrm{e}}-1^{\mathrm{e}}$ av. & B-M 242 \\
\hline \multirow{2}{*}{$Y S^{2} B M$} & $\begin{array}{l}\text { Bastion } \\
(m h f d n)\end{array}$ & $\dot{G} z r-S^{\prime} h f n$ & $?$ & Wqh'l Rym (?) & $5^{e}-4^{e}$ av. & $\begin{array}{l}\text { B-M } 280=R E ́ S \\
2976\end{array}$ \\
\hline & $\begin{array}{l}\text { Bastion } \\
(m h f d n ?)\end{array}$ & Royal & Royal & $Y \underline{t}^{\circ} l S \underline{S} d q$ & $\begin{array}{l}1^{\mathrm{e}} \text { av. } \\
\text { v. }-70\end{array}$ & $\begin{array}{l}\text { B-M } 259=R E ́ S \\
2973\end{array}$ \\
\hline$Y S^{2} H R$ & $\begin{array}{l}\text { Building } \\
(h t b n)\end{array}$ & $?$ & $?$ & $?$ & $8^{\mathrm{e}}$ av. & $\begin{array}{l}\text { MAFRAY- } \\
\text { Malāḥā' } 2\end{array}$ \\
\hline$Y \underline{T}^{\prime} N$ & $\begin{array}{l}\text { Bastion } \\
(m h f d n)\end{array}$ & $S^{2 c} \underline{t} m$ & $G b^{\prime} n$ & $\begin{array}{l}\text { Postérieur à } \\
\text { 'lyf } Y S^{2} r \quad w-b n-s^{1} \quad H f n m \\
\text { Rym }\end{array}$ & $3^{e}-1^{e}$ av. & $\begin{array}{l}\text { B-M } 273=R E ́ S \\
2978\end{array}$ \\
\hline
\end{tabular}




\begin{tabular}{|c|c|c|c|c|c|c|}
\hline$Y^{c} D$ & Maison (byt) & $S^{2} \underline{t} m$ & $G b^{\prime} n$ & Wqh'l Rym & $5^{\mathrm{e}}-4^{\mathrm{e}}$ av. & $\begin{array}{l}\text { Y.90.B.ext } 2= \\
\text { YM 26117 }\end{array}$ \\
\hline$Z B Y N$ & $\begin{array}{l}\text { Bastion } \\
(m h f d n)\end{array}$ & $S^{2} \underline{t} t m$ & $G b^{\prime} n$ & $\begin{array}{l}\text { 'lyf } Y f^{2} r \quad w-b n-s^{1} \quad H f n m \\
\text { Rym }\end{array}$ & $3^{\mathrm{e}}-2^{\mathrm{e}}$ av. & $\begin{array}{l}\text { B-M } 272=R E ́ S \\
3021\end{array}$ \\
\hline \multirow{2}{*}{$Z R B N$} & \multirow{2}{*}{$\begin{array}{l}\text { Bastion } \\
(m h f d n)\end{array}$} & $\begin{array}{l}Y f^{\prime} n \\
D f g n\end{array}$ & $\begin{array}{l}G b^{\prime} n / \\
?\end{array}$ & 'byd' $Y \underline{t}^{c}$ & $5^{\mathrm{e}}$ av. & $\begin{array}{l}\text { B-M } 257=R E ́ S \\
3022\end{array}$ \\
\hline & & $S^{2} \underline{t} m$ & $G b^{\prime} n$ & $\begin{array}{l}\text { 'lyf } Y s^{2} r \quad w-b n-s^{1} H f n m \\
\text { Rym }\end{array}$ & $3^{\mathrm{e}}-2^{\mathrm{e}}$ av. & $\begin{array}{l}\text { B-M } 272=R E ́ S \\
3021\end{array}$ \\
\hline
\end{tabular}




\section{Annexe 3 - Liste des clans et fractions de clan à Barāqish}

\begin{tabular}{|c|c|c|}
\hline Clans & Sous-clans & Offices de membres des sous-clans \\
\hline \multirow{10}{*}{$G B^{3} N$} & Blh & $\begin{array}{l}\text { Chef de Yathill }(k b r Y \underline{t} t) \\
\text { Congrégation de 'Athtar dhu-Yahriq (qhlt ' } \underline{\underline{t} t r} \underline{\underline{d}}-\mathrm{Yhrq})\end{array}$ \\
\hline & $F^{\prime} d$ & \\
\hline & $H d \underline{l l n}$ & \\
\hline & $H f d$ & \\
\hline & $H f n$ & \\
\hline & $R d^{c}$ & Chef de Yathill (?) $(k b r[Y \underline{t} l l(?)])$ \\
\hline & $S^{2} \underline{t} \underline{t}$ & \\
\hline & $Y f^{\prime} n$ & $\begin{array}{l}\text { [Du conseil] des Douze }\left(\underline{d}-\underline{t} n y \text { ' }^{2} r\right) \\
\text { Congrégation de 'Athtar dhu-Yahriq (qhlt 't } \underline{t} t r \underline{d}-Y h r q) \\
\text { Chef de Yathill (?) }(k b r[Y \underline{t} l(?)]) \\
\text { Chef des caravaniers de Ma'in }\left(k b r M^{\prime} n m s \underline{r} n\right)\end{array}$ \\
\hline & Zhrn & Prêtre de de 'Athtar dhu-Yahriq $\left(r s^{2} w^{\prime} \underline{t} t r \underline{d}-Y h r q\right)$ \\
\hline & Zlwmn & Congrégation de 'Athtar dhu-Yahriq (qhlt 't ttr $\underline{d}$-Yhrq) \\
\hline \multirow[b]{2}{*}{ MWQH } & $\dot{G} r b t$ & \\
\hline & Zyrn & Congrégation de 'Athtar dhu-Yahriq (qhlt ' $\underline{\text { ttr }} \underline{\underline{d}}$-Yhrq) \\
\hline$Q_{R N}$ & $H \underline{d} \underline{d k t}$ & \\
\hline \multirow[b]{2}{*}{ YLQZ } & $D b r$ & Chef de la ville de [?] (kbr hgrn [?]) \\
\hline & $M h d \underline{d} r$ & \\
\hline \multirow{2}{*}{${ }^{\prime} L Y^{\prime} L$} & $F^{\prime} m n$ & \\
\hline & $S^{l} y l$ & \\
\hline$?$ & $D f g n$ & Chef des caravaniers de Ma ${ }^{`} i ̄ n\left(k b r M^{`} n\right.$ mșrn) \\
\hline$?$ & Dmrn & \\
\hline$?$ & $D^{\prime} f$ & \\
\hline$?$ & $\dot{G} z r S^{\prime} h f n$ & $\begin{array}{l}\text { Chef des maçons et du transport }(k b r \text { grbn w-nqln) } \\
\text { Chef des serviteurs du roi }\left(k b r^{\prime} d m m l k\right)\end{array}$ \\
\hline$?$ & Hbrr & \\
\hline$?$ & Hrn & \\
\hline$?$ & Mlh & \\
\hline$?$ & Mrn & \\
\hline$?$ & $M^{\prime} f y$ & \\
\hline$?$ & Rwyn & \\
\hline$?$ & $S^{I} y l m$ & Congrégation de 'Athtar dhu-Yahriq (qhlt 't tr $\underline{d}$-Yhrq) \\
\hline$?$ & $S^{2} g^{\prime}$ & Sacrificateur (?) de 'Athtar dhu-Qabḍ ( $\left.s^{2} w^{c}{ }^{c} \underline{t} t r \underline{d}-Q b d \underline{)}\right)$ \\
\hline$?$ & $S^{2} y d$ & Prêtre de de 'Athtar dhu-Yahriq $\left(r s^{2} w^{\prime} \underline{t} t r \underline{d}-Y h r q\right)$ \\
\hline$?$ & $s^{2} m$ & \\
\hline$?$ & 'qrn & \\
\hline$?$ & 'rqn & \\
\hline$?$ & ${ }^{c} s^{I} d n$ & \\
\hline$?$ & $y s^{2} n$ & Congrégation de 'Athtar dhu-Yahriq (qhlt ' $\underline{\text { ttr }} \underline{\underline{d}}$-Yhrq) \\
\hline
\end{tabular}




\section{Annexe 4 - La chronologie des rois de $\mathrm{Ma}^{\text {cīn }}$ revisitée}

\subsection{Revisiter la chronologie royale de $\mathrm{Ma}^{\mathrm{i}} \overline{\mathrm{in}}$}

Pour les besoins de cette étude et l'ancrage des événements rapportés par les inscriptions dans un cadre chronologique, une liste de la succession des rois de Ma 'īn s'avérait nécessaire. La mention épigraphique des souverains est, avec la paléographie, l'un des rares éléments de chronologie que nous offrent les textes. Plusieurs chronologies ont été élaborées (pour n'en citer que les principales : Winnett 1939 ; Albright 1953 ; Pirenne 1956 ; Von Wissmann 1976 ; Arbach 1993 ; Kitchen 1994 ; Bron 1998 ; Arbach 2018).

Aucune n'est pleinement satisfaisante dans le sens où les souverains ne sont pas tous connus, les homonymes ne sont pas aisément distinguables et la filiation des souverains n'est pas toujours renseignée. Tout au plus pouvons-nous restituer des groupes de souverains qu'il n'est pas toujours aisé de positionner les uns par rapport aux autres.

Il est ici apparu nécessaire d'entreprendre un nouveau classement pour deux raisons. La première est qu'un corpus plus large de données est désormais disponible ; la seconde est que la datation absolue du règne d'un souverain majeur, 'byd $Y \underline{t}^{c}$ se précise grâce au croisement des données archéologiques et épigraphiques.

\section{2 'byd 'Yt $^{c}$, roi de $\mathrm{Ma}^{\mathrm{i} i n}$ au $5^{\mathrm{e}}$ siècle av. J.-C.}

L'inscription $R E ́ S 3022$ a été rédigée sous le règne de 'byd' $Y \underline{t}^{c}$ roi de Ma'īn et rapporte une révolte de l'Égypte contre les Mèdes $(M \underline{d} y)$. Rares sont les événements historiques externes renseignés par les inscriptions sudarabiques et ce texte a été l'une des clés dans l'établissement de la chronologie sudarabique. Une littérature abondante est consacrée à ce synchronisme (voir en particulier Lemaire 1996 : 44-47 et références citées).

La date couramment retenue est un règne au milieu du $4^{\mathrm{e}}$ siècle av. J.-C., l'événement de $R E S$ 3022 étant associé à l'expédition d'Artaxerxés III Ochos en Égypte en 343 av. J.-C.

Cette date ne semble toutefois pas devoir être retenue. Une date du $5^{\text {e }}$ siècle av. J.-C. est préférable pour plusieurs raisons.

A. Lemaire a déjà présenté plusieurs arguments dans ce sens (Lemaire 1996 : 45-47) :

- Trois satrapies perses sont mentionnées dans RÉS 3022 : l'Égypte (Mșr), l'Assyrie $\left(" s^{2} r\right.$ ), la Transeuphratène ( $b r N h r n$ ) : la création d'une satrapie de Transeuphratène, distincte de celle d'Assyrie, date de la réorganisation administrative de la région par Xerxés I ${ }^{\text {er }}$, vers 482 av. J.-C. Par ailleurs, la Transeuphratène est rattachée à la Cilicie en 345. La mention de la Transeuphratène, si elle est évoquée en tant que satrapie et non comme aire géographique, indique une période comprise entre 482 et 345.

- L'inscription RÉS 3022 évoque une révolte $(m r d)$ des Égyptiens contre les Mèdes et non une guerre $(d r)$. Cela renverrait plutôt à l'un des cinq événements identifiés par A. Lemaire comme des révoltes avérées ou des périodes de rejet de l'autorité perse (46354 ; 422 ; 414-13; $411 ; 405-398)$.

A. Multhoff a également démontré sur la base d'une analyse philologique et historique précise que les événements rapportés par les inscriptions RÉS 3022, Bauer 5, RÉS 3869 et Demirjian 1 étaient liés les uns aux autres (Multhoff 2019) de sorte que RÉS 3022 évoque très certainement 
une révolte égyptienne de la fin du $5^{\mathrm{e}}$ siècle av. J.-C. et que la guerre entre Chaldéens et Ioniens rapportée par Demirjian 1 renverrait à la guerre d'Euagoras (vers 390-380 av. J.-C.), telle que proposé dans une récente étude (Sørensen \& Geus 2019). A. Multhoff, tout comme A. Lemaire propose donc de dater le règne de 'byd $Y \underline{t}^{\mathrm{c}}$ à la fin du $5^{\mathrm{e}}$ siècle av. J.-C.

Un troisième point semble faire pencher la balance en faveur de cette datation haute : les datations radiocarbones obtenues dans les temples de Barāqish.

La première est un échantillon prélevé au niveau du pavement L110 du temple de 'Athtar (Bar.05.B/2a), dans un contexte de peu postérieur à la construction de l'édifice. L'âge radiocarbone, $2438 \pm 27$, donne la date calibrée de 750-408 cal-BCE (2 sigma) avec une probabilité médiane à -528 (voir en particulier les contributions de A. Agostini, « Il Tempio di 'Athtar dhu-Qabḍ a Barāqish » et de F. Fedele « Radiocarbon determinations 1987-2006... » dans ce volume). La construction de ce temple intervient au cours du règne de $W q h^{\prime} l$ Rym fils de 'byd' $Y \underline{t}^{c}$; à moins d'un effet vieux bois sur l'échantillon, il est peu probable que la construction du temple soit postérieure à 343 av. J.-C., date habituellement proposée pour le règne du père de Wqh'l Rym. Le temple de 'Athtar dhu-Qabḍum fut vraisemblablement bâti à une date plus haute (fin $5^{\mathrm{e}}$-début $4^{\mathrm{e}}$ siècle av. J.-C.), ce qui s'accorderait mieux avec la datation radiocarbone.

Le second échantillon analysé (Y.92.B/138) provient des dépôts inférieurs de la phase B du temple de Nakrah. Nous avons vu que les aménagements de la phase B correspondent certainement aux travaux réalisés sous le règne de 'byd $Y \underline{t}^{c}$ (voir « 5.2.1 - Brn, temple de Nakraḥ à Barāqish »). Son âge radiocarbone est 2380 \pm 50 soit 750-380 cal-BC. Là encore, à moins d'un effet vieux bois, l'échantillon indiquerait des travaux réalisés avant la date de 380 av. J.-C.

Compte tenu des données philologiques, historiques et des datations radiocarbones, le règne de 'byd' $Y \underline{t}^{\mathrm{c}}$ nous semble devoir être remonté à la seconde moitié du $5^{\mathrm{e}}$ siècle. L'événement rapporté par RÉS 3022 intervient à une date comprise entre 463 et 405 . Les datations radiocarbones tendent également à faire remonter des réalisations architecturales datées du règne de son fils $W q h^{\prime} l$ Rym au $5^{\mathrm{e}}$ siècle av. J.-C. Les échantillons ne sont toutefois pas assez nombreux pour en être assuré. Les données s'accordent au moins pour dater celui-ci de la fin du $5^{\mathrm{e}}$-début $4^{\mathrm{e}}$ siècle av. J.-C.

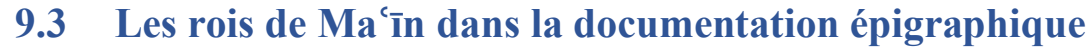

Le tableau ci-dessous propose une chronologie royale où la succession des groupes se fonde sur la graphie, en partant du principe que le style paléographique D de J. Pirenne est postérieur au style E, tel qu'elle l'admettait elle-même. Lorsque les inscriptions propres à certains groupes relèvent de mêmes types paléographiques, leur mise en ordre n'est pas toujours assurée. Dans ce cas, ils sont présentés accolés de la mention "groupes interchangeables », en laissant la possibilité de leur succession ouverte.

Certains groupes présentant une graphie identique peuvent être calés les uns par rapport aux autres grâce au contexte de découverte de l'inscription ou à des éléments prosopographiques. Cela reste rare.

Les datations absolues se fondent sur les datations radiocarbones obtenues sur le site de Barāqish (la graphie $\mathrm{C}$ est contemporaine de constructions datées du $6^{\mathrm{e}}$ siècle av. J.-C. ; la 
graphie E2-E3, celle des règnes de 'byd' $Y \underline{t}^{\mathrm{c}}$ et $W q h^{\prime} l \mathrm{Rym}$, de la seconde moitié du $5^{\mathrm{e}}$ - première moitié du $4^{\mathrm{e}}$ siècle av. J.-C.). 


\section{Tableau 9 : Liste des rois de Ma īn classés par ordre chronologique}

\author{
v. VIII' ${ }^{\mathrm{e}}$ s. av. J.- 'byd' \\ C.

\begin{tabular}{|c|c|c|}
\hline & 'byd' $w-Y \underline{t}^{\prime \prime} l$ & Ma'in 39 et 43 \\
\hline & $Y \underline{t}^{\prime \prime} l w-H y w$ & $\mathrm{Ma}^{\prime} \overline{i n} 18$ et 33 \\
\hline v. -710 & $Y \underline{t}{ }^{\prime \prime} l w-S b h m$ & YM 2009 \\
\hline
\end{tabular} \\ Ma'īn 112 \\ YM 2009
}

.

v. VII ${ }^{\mathrm{e}}$ s. av. J.- Nbt'l

C.

v. $-700 /-650 \quad$ 'byd' $Y f^{2}{ }^{2} b n N b t^{\prime} l$

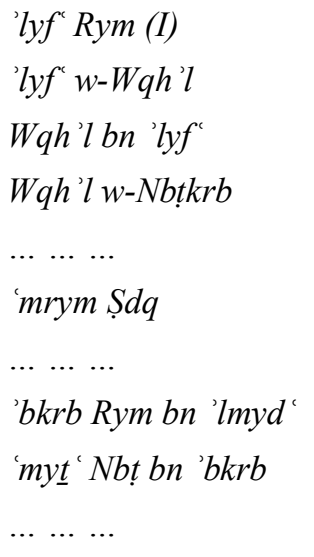

v. $\mathrm{VI}^{\mathrm{e}}$ s. av. J.-C. Hlkrb $S d q b n$ 'byd'

$H \mathrm{fn}^{[\mathrm{m}]} \mathrm{Yt}^{\prime}$ bn $\mathrm{Hlkrb}$

$H f n^{m} Y \underline{t}^{\prime} w$-' $l y f^{\prime} R y m$

'lyf' Rym (II)

'lyf' Rym (II) w-bn-s' $H w f^{\prime} \underline{t} t$

........

v. $V^{\mathrm{e}}$ s. av. J.-C. $W$ hh' $^{\prime} S d q b n^{\prime} l y f^{\prime}(?)$

$$
\begin{aligned}
& \cdots \cdots \cdots \\
& ' l y f^{\prime} Y \underline{t}^{\prime} \\
& ' l y f^{\prime} Y \underline{t^{\prime}} w \text {-' } b y d^{\prime}
\end{aligned}
$$

Après $\quad-463$ 'byd' $Y \underline{t}$

Avant -405

byd 'Y ${ }^{`} w-b n-s^{l} W q h^{\prime} l$ Rym

v. IV's. av. J.-C. Wqh'l Rym bn 'byd'

YM 30135

Shaqab 2

Ma'īn 15 et 17

RÉS 2947

Ma'in 7

Ma'īn 2 Y.92.B.A 27 $\leftarrow \rightarrow \underline{t}^{\prime \prime} m r$ [Byn] w-Didr'ly [Drh $\left.m k r b \quad S^{\prime} b^{\prime}\right]+Y q h m l k \quad[m l k$ $\left.N s^{2} n\right]$

Ma'īn 107 et 108

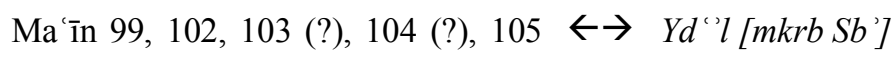

(?), 108 (?), 109 (?), 110 (?), 113 (?)

Shaqab 6, Gajda 2001, al-Jawf 04.30

Ma '̄in 101, RÉS 4834 (?)

Schm/Samsara 3

YM 26106 et 28488

$R E ́ S 2980=$ Shaqab 19

$\leftarrow \rightarrow$ Attaque sabéenne contre Ma īn et Yathill (RÉS 3943)

Ma'īn 82, 84, 85, 86, 88, 89, 100

DhM 393, Gl 312, Ma'in 9 et 10

Ma 'īn 90, RÉS 3012

$R E ́ S 2789=\mathrm{Ma}^{\prime}$ in 13

GOAM 315, Ma'īn 1 et 8, RÉS 2959, $2972+2970+2971+2971$ bis, 3006, 3022, 3029, Y.92.B.A 21+30,

B-M 122 (?), Bauer 5, RÉS 2929+2941+2945+2946, 2942, 2944, 2952, 3012, 3013

B-M 68, Bron Semitica 55.1 (?), RÉS 3005, 3055, Shaqab 18, Y.05.B.B.13, YM 26117
Révolte des Égyptiens contre les Mèdes $' s^{1} m^{\prime}$ Dbyn bn Mlkkrb + S'hrm 'Thn bn Yd'l [mlk Hḍrmt] 


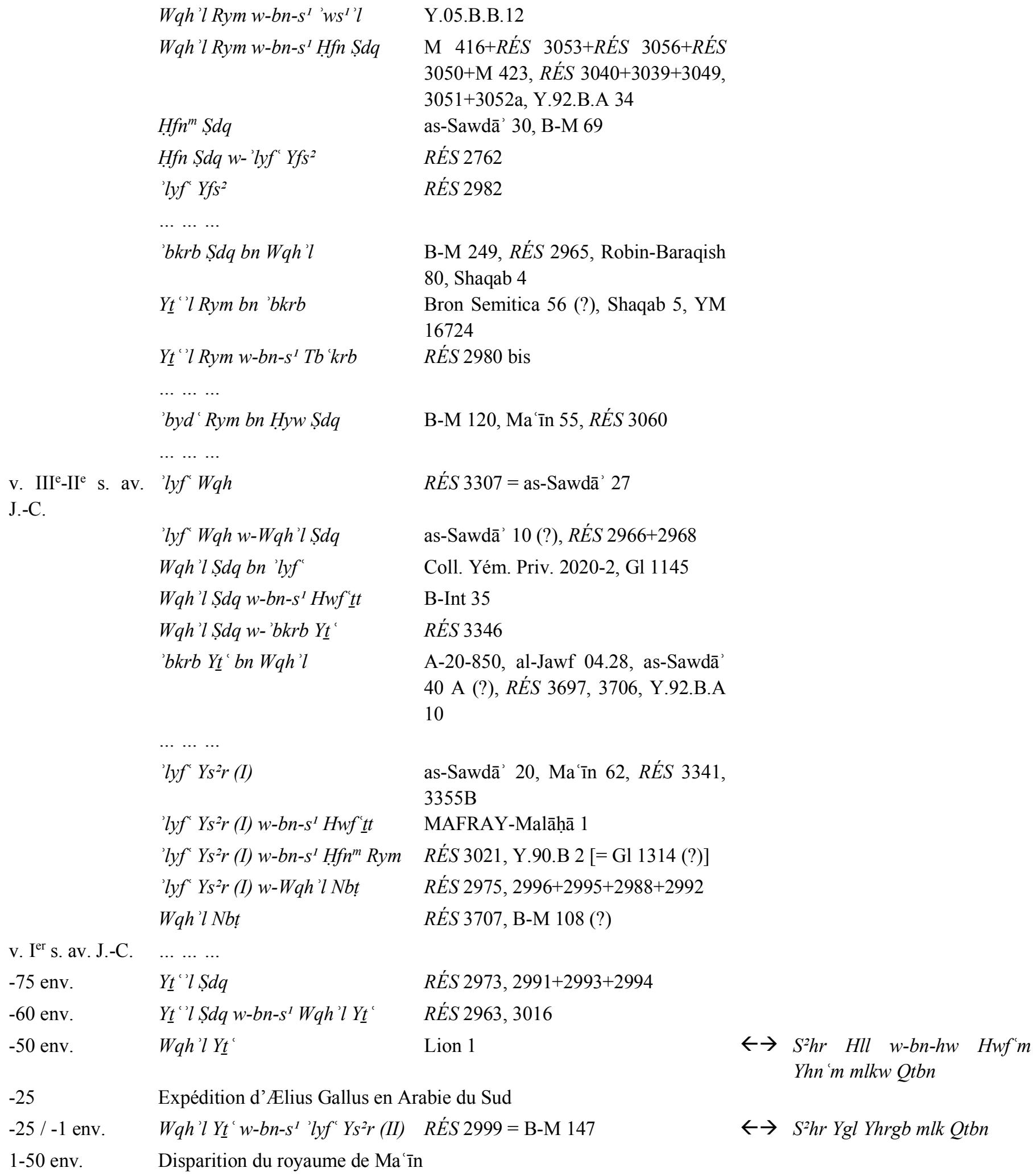




\section{Références bibliographiques}

\subsection{Sources}

al-Așma ${ }^{c} \overline{1}$, al-Așma ìyyāt :

al-Așma $\overline{1}, A b \bar{u}$ Sa'īd 'Abd al-Malik b. Qarīb b. 'Alī b. Așma'. Al-așma iyyāt. Edited by A.M.

Shākir \& A.M. Hārūn, Cairo: Dār al-Ma ārif, $7^{\text {th }}$ edition, 1993.

al-Hamdān̄̄, al-Iklīl :

al-Hamdān̄̄, al-Ḥasan ibn Aḥmad ibn Ya qūb Ibn al-Ḥà̄ik. The Antiquities of South Arabia,

Being a Translation from the Arabic with Linguistic, Geographic, and Historic Notes of the

Eighth Book of al-Hamdānī's al-Iklil. Edited by N.A. Faris. Princeton, 1938. Yāqūt, Mu'jam al-buldān, 1 :

Yāqūt ibn 'Abd Allāh al-Hamawī. Jacut's geographisches Wörterbuch, aus den Handschriften zu Berlin, St. Petersburg und Paris. Erster Band I _ ث. Edited by Ferdinand Wüstenfeld. Vol.

3. Leipzig : Brockhaus, 1866.

\subsection{Références}

Agostini, A. 2011. Two new inscriptions from the recently excavated temple of Athtar dhūQabd in Barāqish (Ancient Minaean Yathill) Arabian Archaeology and Epigraphy 22 (May 2011) : 48-58.

- 2012. New perspectives on Minaean expiatory texts. Proceedings of the Seminar for Arabian Studies 42 : 1-12.

— 2015. The excavation of the temple of 'Athtar dhū-Qabḍ in Barāqish. Stratigraphic data and historical reconstruction. Proceedings of the Seminar for Arabian Studies 45 : 1-14.

Albright, F.P. 1953. The Chronology of the Minaean Kings of Arabia. Bulletin of the American School of Oriental Research $129: 20-24$.

ANTONINI, S. 1988. Nuovi incensieri iscritti yemeniti. Oriens Antiquus 27 : 133-41.

— 1999. Statuettes from the temple of Nakrah at Barāqish Arabian Archaeology and Epigraphy $10: 58-68$.

- 2005. Alcune stele inedite dal Jawf (Yemen), in A.V. Sedov \& I.M. Smiljanskaja (ed.) Arabia Vitalis. Arabskij Vostok, islam, drevnjaja aravija. Sbornik statej posvjaščennyj 60-letiju V. V. Naumkina: 308-13. Moscow: Rossijskaja Akademija Nauk / Moskovskij Gosudarstvennyj Universitet.

Antonini, S. \& A. Agostini. 2010. A Minaean Necropolis at Barâqish (Jawf, Republic of Yemen). Preliminary Report of the 2005-2006 Archaeological Campaigns. Rome: IsIAO.

ARBACH, M. 1993. Lexique madhābien: comparé aux lexiques sabéen, qatabanite et hadramawtique. Aix-en-Provence : M. Arbach.

- 1994. Inscriptions sudarabiques. Raydān $6:$ 5-16.

- 2011. La découverte du temple intra-muros de Nakrah à Ma ${ }^{i ̄ n}$, l'antique Qarnā. Arabian Archaeology and Epigraphy 22 : 201-14.

- 2018. L'Arabie du Sud: de la Cité-État au royaume (VIII ${ }^{e}-V I^{e}$ s. av. J.-C.). Mémoire d'Habilitation à Diriger des Recherches, Paris : Université Paris 1.

Arbach, M. \& R. Audouin. 2007. San 'à’ National Museum. Part II. Collection of epigraphic 
and archaeological artifacts from al-Jawf sites. Sanaa National Museum, 2. Sanaa: UNESCO - FSD - CEFAS.

ARBACH, M. \& I. RosSI. 2012. From city-state to kingdom : History and chronology of Ma īn between the 8th and the 6th century BC. Orientalia $81.4: 318-39$.

Arbach, M. \& J. SchietTeCATte. 2017. Premiers échos de l'expédition romaine d'Ælius Gallus dans la documentation sudarabique. Comptes rendus des séances de l'Académie des Inscriptions \& Belles-Lettres avril-juin 2017 : 675-700.

Avanzini, A. 1995. As-Sawdä'. Inventario Delle Iscrizioni Sudarabiche, T. 4. Paris-Rome : Académie des Inscriptions et Belles-Lettres - Istituto per Il Medio ed Estremo Oriente.

- 2004. Corpus of South Arabian Inscriptions I-III. Qatabanic, Marginal Qatabanic, Awsanite Inscriptions. Arabia Antica 2. Pise: Edizioni Plus.

- 2016. By land and by sea: a history of South Arabia before Islam recounted from inscriptions. Rome : L'Erma di Bretschneider.

BAuer, G.M. \& A. Lundin G. 1998. Užnaâ Araviâ. Pamâtniki drevnej istorii i kul'tury. vypusk 2, čast' 2, Èpigrafičeskie pamâtniki drevnego Jemena. Saint Petersburg: Centr 'Peterburgskoe vostokovedenie'.

Breton, J.-F. 1994. Archäologische Berichte aus dem Yemen VIII. Les fortifications d'Arabie Méridionale du 7e au le siècle avant notre ère. Mainz : Verlag Philipp von Zabern.

Breton, J.-F., Ch.J. Robin. \& R. Audouin. 1979. Prospection archéologique et épigraphique de la Mission Archéologique Française au Yémen du Nord (oct-déc. 1978). Syria LVI : 417-27.

Bron, F. 1998. Inventaire des inscriptions sudarabiques. Tome 3 : Ma'in. Paris-Rome: Académie des Inscriptions et Belles-Lettres-Istituto Italiano per l'Africa e l'Oriente, Diffusion de Boccard - Diffusion Herder.

De Maigret, A. 1991a. The Excavations of the Temple of Nakrah at Baraqish (Yemen). Proceedings of the Seminar for Arabian Studies 21 : 159-72.

- 1991b. Gli scavi della Missione Archeologica nella citta minea di Barâqish. Rome.

- 1993. La seconda campagna di scavi della Missione Archeologica italiana a Barâqish. Conferenze IsMEO 6. Rome.

- 2004. Barâqish, Minaean Yathill. Excavation and Restoration of the Temple of Nakrah. YICAR Papers, 1. Naples : Il Torcoliere, Universita degli Studi di Napoli 'L'Orientale'. — 2010. A Sabaean stratigraphy from Barāqish Arabia 4 : 67-96.

De Maigret, A., G.M. Bulgarelli., L. Costantini., M. Cucarzi., F. Di Mario., F.G. Fedele., V. Francaviglia., G. Gnoli., B. Marcolongo., A.M. Palmieri., G. Pettinato., U. Scerrato., M. Tosi. \& G. Ventrone. 1986. Archaeological Activities in the Yemen Arab Republic, 1986. East and West 36 : 377-470.

De Maigret, A. \& Ch. J. RoBin. 1993. Le temple de Nakrah à Yathill (Barâqish), Yémen. Résultats des deux premières campagnes de fouilles de la mission italienne. Comptes rendus des séances de l'Académie des Inscriptions \& Belles-Lettres 1993 : 427-96.

FAKHRY, A. 1951. An archaeological Journey to Yemen (March-May 1947). 3 vols. Cairo: Government Press.

FEDELE, F.G. 2010. Barāqish, over-wall excavations 2005-2006 : stratigraphy, environment and economy of the Sabaean-Islamic sequence. Arabia 4 (2007-2010) : 97-161.

GARBINI, G. 1974. Iscrizioni sudarabiche, vol. 1. Iscrizioni minee. Publicazioni Del Seminario 
Di Semitica, Ricerche X. Naples : Istituto Orientale di Napoli.

GNOLI, G. 1993. Shaqab al-Manassa. Inventario delle iscrizioni sudarabiche, tome 2. Paris / Rome : Académie des Inscriptions et Belles-Lettres / Istituto per Il Medio ed Estremo Oriente.

- 1996. Il sincronismo Mineo-Persiano, in C. Robin \& I. Gajda (ed.) Arabia antiqua, Early Origins of South Arabian States, Proceedings of the First International Conference on the Conservation and Exploitation of the Archaeological Heritage of the Arabian Peninsula : 23-34. Serie Orientale Roma LXX, 1. Rome : IsMEO.

GNOLI, G. \& Ch.J. RoBIN. 1992. Nouveaux documents sabéens de Barāqish. Yemen. Studi archeologici, storici e filologici sull'Arabia meridionale 1 : 93-98.

HaleVy, J. 1872. Rapport sur une mission archéologique dans le Yémen. Journal Asiatique, 6e série XIX, jv-ju 1872 : 5-98, 129-266 et 489-547.

HeINRICHS, W. 1998. Al-Sharqī b. al-Quțāmī and his etiologies of proverbs, in S. Leder (ed.) Story-telling in the framework of non-fictional Arabic literature : 282-308. Wiesbaden : Harrassowitz.

Kitchen, K.A. 1994. Documentation for Ancient Arabia, Part I. Chronological framework and historical Sources. Liverpool : Liverpool University Press.

Lemaire, A. 1996. Histoire du Proche-Orient et chronologie sudarabique avant Alexandre, in C. Robin \& I. Gajda (ed.) Arabia antiqua, Early Origins of South Arabian States, Proceedings of the First International Conference on the Conservation and Exploitation of the Archaeological Heritage of the Arabian Peninsula : 35-48. Serie Orientale Roma LXX, 1. Rome : IsMEO.

MarColongO, B. 1997. Les systèmes irrigués de Barāqish, in Ch. Robin \& B. Vogt (ed.) Yémen, au pays de la reine de Saba'. Exposition présentée à l'Institut du Monde Arabe du 25 oct 1997 à fév. 1998 : 78. Paris : Flammarion.

Müller, D.H. von. 1896. Athrula, in Paulys Realencyclopädie der Classischen Altertumswissenschaft, II, 2 : 2071-72.

Müller, D.H. von. \& N. RhodoKANAKIS. (ed.) 1913. Eduard Glasers Reise nach Marib. Sammlung Eduard Glaser I. Vienne : Alfred Hölder.

MüllER, W.W. 1982. Bemerkungen zu einigen von der Yemen-Expedition 1977 des Deutschen Archäologischen Instituts aufgenommenen Inschriften aus dem Raum Mārib und Barāqiš, in J. Schmidt (ed.) Archäologische Berichte aus dem Yemen I : 129-34. Mainz : Verlag Philipp von Zabern.

MulthofF, A. 2019. Merchant and marauder-The adventures of a Sabaean clansman. Arabian Archaeology and Epigraphy, 1-24.

NĀMĪ, K.H. 1954. Nuqūš hirbat Barāqiš ‘alā ḍaw’majmū'at Muhammad Tawfīq (1). Majallat kulliyat al-'ādāb $16: 1-21$.

- 1955. Nuqūš hirbat Barāqiš ‘alā ḍaw’majmū'at Muḥammad Tawfīq (2). Majallat kulliyat al'ā $\bar{a} b 17: 1-22$.

— 1956. Nuqūš hirbat Barāqiš ‘alā ḍaw’majmū'at Muḥammad Tawfīq (3). Majallat kulliyat al'àdāb $18: 1-36$.

- 1957. Nuqūš hirbat Barāqiš ‘alā ḍaw’majmū'at Muḥammad Tawfīq (4). Majallat kulliyat al'àdāb 19 : 93-124.

PIRENNE, J. 1956. Paléographie des inscriptions sud-arabes. Contribution à la chronologie et 
à l'histoire de l'Arabie du Sud antique. I, Des origines jusqu'à l'époque himyarite. Verhandelingen van de Koninklijke Vlaamse Academie Voor Wetenschappen, Letteren En Schone Kunsten van België. Klasse Der Letteren 26. Brussel : Paleis der Academiën. http ://www.sudoc.fr/023459158.

- 1961. Le royaume sud-arabe de Qatabān et sa datation d'après l'archéologie et les sources classiques jusqu'au Périple de la Mer Érythrée. Bibliothèque du Muséon, 47. Louvain. Reimer, P.J., E. BARD., A. BAyliss., J.W. Beck., P.G. Blackwell., C.B. RAMSeY., C.E. Buck., H. Cheng., R.L. EdWARds., M. Friedrich., P.M. Grootes., T.P. Guilderson., H. Haflidason., I. Hajdas., C. Hatte., T.J. Heaton., D.L. Hoffmann., A.G. Hogg., K.A. Hughen., K.F. Kaiser., B. Kromer., S.W. Manning., M. Niu., R.W. Reimer., D.A. Richards., E.M. Scott., J.R. Southon., R.A. Staff., C.S.M. TURNEY. \& J. VAN DER PLICHT. 2013. IntCal13 and Marine13 Radiocarbon Age Calibration Curves 0-50,000 Years cal BP. Radiocarbon 55 : 1869-87.

RoBIN, Ch.J. 1979a. À propos des inscriptions in situ de Barāqish, l'antique YTL (NordYémen). Proceedings of the Seminar for Arabian Studies 9 : 102-12.

- 1979b. Mission archéologique et épigraphique française au Yémen du Nord en automne 1978. Comptes rendus des séances de l'Académie des Inscriptions \& Belles-Lettres 1979 : 174-201.

— 1984. La cité et l'organisation sociale à Ma`īn : l'exemple de YTL (aujourd'hui Barāqish), in A.T. al-Ansary (ed.) Studies in the history of Arabia, vol. 2 : Pre-Islamic Arabia: 157-64. Riyadh : King Saud University Press.

- 1987. Trois inscriptions sabéennes découvertes près de Barāqish (RAY). Proceedings of the Seminar for Arabian Studies 17 : 165-77.

- 1988. Quelques observations sur la date de construction et la chronologie de la 1ère digue de Marib d'après les inscriptions. Proceedings of the Seminar for Arabian Studies 18 : 95-113.

- 1991. La pénétration des Arabes nomades au Yémen, in Ch.J. Robin (ed.) L'Arabie antique de Karib'īl à Mahomet. Nouvelles données sur l'histoire des Arabes grâce aux inscriptions: 71-88. Revue des Mondes Musulmans et de La Méditerranée 61 (1991). Aix-en-Provence : Edisud.

- 1996. Les premiers États du Jawf et la civilisation sudarabique, in Ch. J. Robin \& I. Gajda (ed.) Arabia antiqua. Early Origins of South Arabian States, Proceedings of the First International Conference on the Conservation and Exploitation of the Archaeological Heritage of the Arabian Peninsula: 49-65. Serie Orientale Roma LXX, 1. Rome : IsMEO.

- 1998. La fin du royaume de Ma'īn. Res Orientales (Parfums d'Orient) XI : 177-88.

- 2016a. La chronologie de Qatabān. Premiers repères, in A. de Maigret \& Ch.J. Robin (ed.)

Tamna' (Yémen). Les fouilles italo-françaises. Rapport final: 53-80. Orient \& Méditerranée 20. Paris : De Boccard.

- 2016b. Inventaire relatif aux souverains de Qatabān, in A. de Maigret \& Ch.J. Robin (ed.)

Tamna' (Yémen). Les fouilles italo-françaises. Rapport final : 81-98. Orient \& Méditerranée 20. Paris : De Boccard.

Robin, Ch. J., S. Antonini. \& F. Bron. 2007. Nouveautés épigraphiques. Arabia 3 : 273-280. RoBIN, Ch. J., J.-F. BRETON. \& J. RYCKMANS. 1981. Le sanctuaire minéen de NKRḤ à Darb 
aṣ-Șabī (environs de Barāqish), Rapport préliminaire. Raydān 4 : 249-62.

RoBIN, Ch. J., J.-F. BRETON. \& J. RYCKMANS. 1988. Le sanctuaire minéen de NKRḤ à Darb aṣ-Ṣabī (environs de Barāqish), Rapport préliminaire, (seconde partie). Étude des Inscriptions. Raydān $5:$ 91-158.

Robin, Ch. J. \& A. DE MAIGRET. 2009. Le royaume sudarabique de Ma'īn : nouvelles données grâce aux fouilles italiennes de Barāqish (l'antique Yathill). Comptes rendus des séances de l'Académie des Inscriptions \& Belles-Lettres 153 : 57-96.

Schiettecatte, J. 2011. D'Aden à Zafar. Villes de l'Arabie du Sud préislamique. Orient et Méditerranée, Archéologie, 6. Paris : De Boccard.

SørEnSEN, S.L. \& K. GeUS. 2019. A Sabaean eyewitness to the war of Euagoras against the Persians. Synchronising Greek and ancient South Arabian sources. Zeitschrift für Papyrologie und Epigraphik 209 : 196-204.

Von Wissmann, H. 1976. Die Geschichte des Sabäerreichs und der Feldzug des Aelius Gallus, in H. Temporini \& W. Haase (ed.) Aufstieg und Niedergang der römischen Welt : 308544. II. Principat, 9. Band, 1. Halbband. Berlin-New-York : de Gruyter.

WINNETT, F.V. 1939. The place of the Mineans in the History of pre-Islamic Arabia. Bulletin of the American School of Oriental Research 73, Feb. 1939 : 3-9.

\section{Caption}

Figure 1 - Barāqish - Localisation des monuments attestés par la documentation épigraphique dans l'antique cité de Yathill et périodisation des cinq phases principales de construction (infographie J. Schiettecatte / plan M. Mascellani 2007, F. Fedele 2019 - MAIRY). 